\title{
Preparing for action : a behavioral and pupillometric study
}

Citation for published version (APA):

Moresi, S. M. J. (2009). Preparing for action : a behavioral and pupillometric study. [Doctoral Thesis, Maastricht University]. Maastricht University. https://doi.org/10.26481/dis.20090416sm

Document status and date:

Published: 01/01/2009

DOI:

10.26481/dis.20090416sm

Document Version:

Publisher's PDF, also known as Version of record

\section{Please check the document version of this publication:}

- A submitted manuscript is the version of the article upon submission and before peer-review. There can be important differences between the submitted version and the official published version of record.

People interested in the research are advised to contact the author for the final version of the publication, or visit the DOI to the publisher's website.

- The final author version and the galley proof are versions of the publication after peer review.

- The final published version features the final layout of the paper including the volume, issue and page numbers.

Link to publication

\footnotetext{
General rights rights.

- You may freely distribute the URL identifying the publication in the public portal. please follow below link for the End User Agreement:

www.umlib.nl/taverne-license

Take down policy

If you believe that this document breaches copyright please contact us at:

repository@maastrichtuniversity.nl

providing details and we will investigate your claim.
}

Copyright and moral rights for the publications made accessible in the public portal are retained by the authors and/or other copyright owners and it is a condition of accessing publications that users recognise and abide by the legal requirements associated with these

- Users may download and print one copy of any publication from the public portal for the purpose of private study or research.

- You may not further distribute the material or use it for any profit-making activity or commercial gain

If the publication is distributed under the terms of Article $25 \mathrm{fa}$ of the Dutch Copyright Act, indicated by the "Taverne" license above, 
PREPARING FOR ACTION

A BEHAVIORAL AND PUPILLOMETRIC STUDY 
ISBN: 978-90-814049-1-4

Copyright, 2009, Sofie M.J. Moresi, Maastricht University, Department of Human Movement Sciences and School of Mental Health and Neurosciences, Division Brain \& Cognition, Faculty of Health, Medicine and Life Sciences, PO Box 616, 6200 MD Maastricht, The Netherlands.

Cover Picture: Sofie Moresi

Cover Design: Bilbo Schickenberg \& Sofie Moresi

Lay out: Sofie Moresi

Printed by: Ipskamp Drukkers Enschede 


\title{
PREPARING FOR ACTION \\ A BEHAVIORAL AND PUPILLOMETRIC STUDY
}

\author{
PROEFSCHRIFT \\ Ter verkrijging van de graad van doctor \\ aan de Universiteit Maastricht, \\ op gezag van de Rector Magnificus, \\ Prof. Mr. G.P.M.F Mols, \\ volgens besluit van het College van Decanen, \\ in het openbaar te verdedigen \\ op donderdag 16 april 2009 om 14.00 uur. \\ door
}

Sofie Michèle Joseph Moresi 
Promotores:

Prof. Dr. J. Jolles (Vrije Universiteit Amsterdam)

Prof. Dr. H. Kuipers

Copromotores:

Dr. J.J. Adam

Dr. P. W.M. van Gerven

Beoordelingscommissie:

Prof. Dr. H. Kingma (voorzitter)

Dr. F. Smulders

Prof. Dr. W.J. Riedel

Prof. Dr. M.W. van der Molen (Universiteit van Amsterdam)

Prof. Dr. W.B. Verwey (Universiteit Twente)

Paranimfen:

Katarina Fredriksson

Marie-Ange Somers 


\section{CONTENTS}

$\begin{array}{lll}1 & \text { Introduction } & 7\end{array}$

2 Pupil dilation in response preparation 27

3 Response preparation with adjacent and overlapped hands 43

4 Response preparation with valid and invalid cues 63

5 Response preparation with static and moving hands 85

6 Response preparation within and between hands in older age 105

7 Epilogue 123

$\begin{array}{ll}\text { Summary } & 134\end{array}$

$\begin{array}{ll}\text { Samenvatting } & 138\end{array}$

Woorden van Dank 142

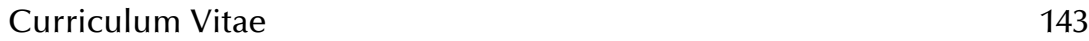

$\begin{array}{ll}\text { Publications } & 144\end{array}$ 

INTRODUCTION

CHAPTER 1

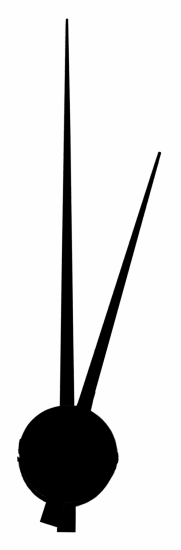


In daily life, people often make use of visual cues. A good example is the orange traffic light, which prepares one to either move on or to stop. Thus, a traffic light turning orange may stimulate preparatory activities, which will allow car drivers to rapidly detect the forthcoming red light and, if necessary, to stop the car immediately. In general, people perform faster when they are presented with a cue that indicates a future event. This thesis focuses on the preparatory processes that enable such a speeded action.

In particular, we examined preparatory processes that underlie the handadvantage, a robust phenomenon in finger-cuing studies. This phenomenon is the reaction time (RT) benefit that occurs when preparing two fingers on one hand as opposed to preparing two fingers on two hands. A recent account of the hand-advantage is the Grouping Model of finger preparation (Adam et al., 2003b, 2005), which is based on two assumptions, the grouping and the processing assumption. The current thesis contains five studies that evaluated these assumptions. Apart from recording RT, we also measured pupil size to provide a more direct, independent and continuous measure of processing load associated with response preparation. Before elucidating the Grouping Model of finger preparation and its assumptions, we briefly describe several general features of response preparation.

\section{FEATURES OF RESPONSE PREPARATION}

In an extensive review of response preparation, Jennings and Van der Molen (2005) described several features of response preparation that are of special importance to our studies.

First, response preparation is effortful and cannot be maintained for a very long time. In fact, the maximum time that optimal preparation can be maintained varies between 0.5 and $3 \mathrm{~s}$, depending on certain task characteristics. Thus, optimal preparation seems to be a time-limited state, which might depend on effort and fatigue. Second, effective preparation requires inhibition of irrelevant responses that are inadequate for the task at hand. Third, there is disparity between different indices of preparation, such as pupil size, heart rate, and cortical measures, because they poorly correlate with each other and with RT. This disparity emphasizes that different processes act to prepare the individual for an expected action. Fourth, there is the multilevel feature, which means that preparation involves top-down and bottom-up processing. Top-down factors include task set and expectations. 
Bottom-up factors include frequency and recency of prior events (i.e., the greater influence of most recent stimulus occurrence). A fifth and final feature of response preparation is that it is action-oriented, which means that the efficiency and effectiveness of preparation depend on the number and compatibility of anticipated actions. For this reason, preparation can be modulated by cues that provide information on an action to be performed. Appropriate cuing reduces complexity and speeds up preparation. A task that incorporates all these features of response preparation is the finger-cuing task, which is described in the next section.

\section{THE FINGER-CUING TASK}

A useful approach to study preparatory processes in perception and action is to provide people with advance information about an upcoming stimulus or response. We used a specific variant of Rosenbaum's (1980) movement precuing task, namely the finger-cuing task devised by Miller (1982). In Miller's finger-cuing paradigm, spatial cues provide advance information about a subset of two possible finger responses, thus allowing a process of selective response preparation, which makes the task action-oriented (cf. Jennings \& Van der Molen, 2005). Participants are forewarned about the location of a required response or, more precisely, about a particular subset of possible responses. Typically, participants respond to horizontally arranged stimuli by pressing spatially compatible keys with the index or middle finger of one of the two hands. The visual display consists of three horizontal rows of symbols, which represent warning stimulus, cue, and target stimulus respectively (see Figure 1.1). The warning stimulus consists of four plus signs, indicating the four possible stimulus-response locations. The cue, which follows a fixed delay, consists of two plus signs, indicating a subset of two possible finger responses. After a certain preparation interval, which may vary between $100 \mathrm{~ms}$ and $5 \mathrm{~s}$, the target stimulus is presented, which is a single plus sign that indicates the required response.

The function of the cue is to provide advance information about a subset of two possible responses. That is, the cue transforms the original four-choice reaction task into a two-choice reaction task. Four cue or preparation conditions are distinguished. In the hand-cued condition, the cue specifies two fingers on the same hand (e.g., the left-index finger and the left-middle finger). In the finger-cued condition, the cue specifies the same fingers on different hands (e.g., the two index fingers). In the neither-cued condition, 
the cue specifies different fingers on different hands (e.g., the left-middle and right-index fingers). Also, an uncued condition is included, which provides no advance information and thus precludes selective preparation of any combination of two finger responses. This is a necessary control condition, because it leaves the basic, four-choice task unaltered. Since two-choice responses normally yield shorter RTs than four-choice responses (Hick, 1952; Hyman, 1953), cue effectiveness is inferred from a significant RT advantage for the two-choice cue conditions (i.e., hand-cued, finger-cued, and neithercued) over the four-choice (uncued) control condition.

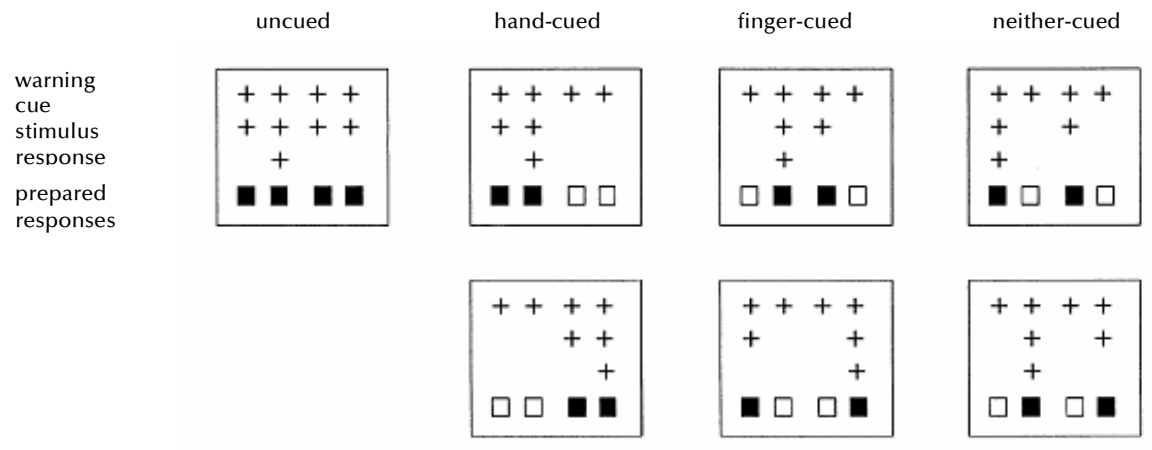

Figure 1.1. Schematic representation of the finger-cuing task and the four preparation conditions (Miller, 1982).

The robust finding from the finger-cuing paradigm is a pattern of differential cuing benefits relative to the uncued condition: RTs are shortest for the handcued condition and longest for the neither-cued condition, with RTs for the finger-cued condition being intermediate (Proctor \& Reeve, 1986, 1988; Reeve \& Proctor, 1984, 1990). This pattern of differential cuing benefits is primarily apparent at short preparation intervals (intervals shorter than 1,500 ms), while longer preparation intervals produce a pattern of equivalent cuing benefits. Thus, with sufficient preparation time, all possible pairs of finger responses can be selected and prepared to the same degree (Proctor \& Reeve, 1986, 1988; Reeve \& Proctor, 1984, 1990). This suggests that response preparation is an effortful process (cf. Jennings \& Van der Molen, 2005) and that different cues may require different levels of effort. 
The pattern of differential cuing benefits has been accounted for by two theories. The salient-features coding principle of Proctor and Reeve (1986, 1988; Proctor et al., 1992; Reeve \& Proctor, 1990) explains the pattern of differential cuing benefits in terms of the saliency of spatial locations. The Grouping Model (Adam et al., 2003b, 2005) attributes the abovementioned pattern to a combination of processes occurring in different information processing loci. These two theories are described in detail in the following sections.

\section{THE SALIENT-FEATURES CODING PRINCIPLE}

According to the salient-features coding principle of Proctor and Reeve (1986, 1988; Proctor et al., 1992; Reeve \& Proctor, 1990), stimulus and response sets are coded in terms of salient features of each, with response selection occurring fastest when salient features of the respective sets correspond. The salient-feature coding principle explains the pattern of differential cuing benefits in terms of a hierarchy of saliency for spatial locations (Reeve \& Proctor, 1990). With the left-right spatial distinction being the most salient feature, there is an advantage for the hand-cued condition (preparing fingers on one hand) over the finger- and neither-cued condition (preparing fingers on two hands). Using an overlapped hand placement (that is, fingers from each hand alternating on the response keys), Reeve and Proctor (1984) showed a benefit for the two left- and rightmost cued locations and not for two fingers of the same hand. Thus, according to Reeve and Proctor (1984, 1990; Proctor \& Reeve; 1986), there is no motoric hand-advantage, but rather a spatial "left-right" advantage.

\section{THE GROUPING MODEL}

The Grouping Model (Adam et al., 2003b, 2005), which is an extension of the salient-features coding principle, is a recent account of the pattern of differential cuing benefits. In the literature, the pattern of differential cuing benefits has been attributed to three different loci: the motor system (Miller, 1982, 1985, de Jong et al., 1988, Leuthold et al., 1996, 2004), stimulus-response translation (Reeve \& Proctor, 1984, 1990), and perceptual encoding (Adam, 1994). In contrast to most previous studies, which focused on and manipulated one specific locus, the Grouping Model considers influences from perceptual, central, and motoric factors. Thus, the Grouping Model 
suggests that the pattern of differential cuing benefits is the combined result of several dynamic and often interacting processes, including perceptual, central, and motoric processes.

Optimal performance in the finger-cuing task requires that cue encoding processes reduce the number of stimulus-response alternatives from four to two. In other words, the functional significance of the cue is to get rid of the irrelevant members in the stimulus and response set, and thus to reduce uncertainty. Consequently, the cue can be considered to direct a process of subgroup making-that is, a process of stimulus- and response-set reconfiguration -whereby the internal representation of the task is simplified (Adam et al., 2003b). But how is this change from a 4- to a 2- element reaction time task achieved?

Visual Buffer

Responses

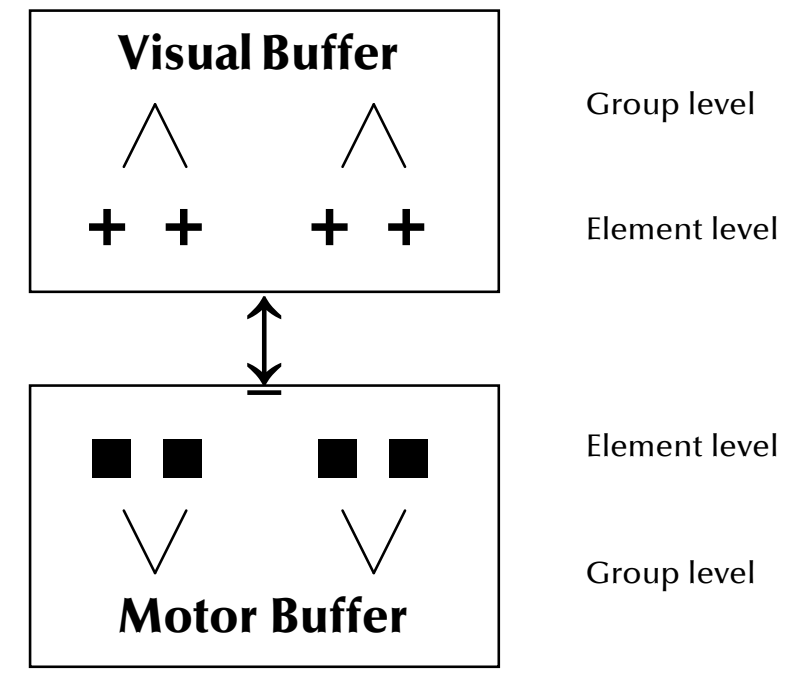

Figure 1.2. Sketch of the representational assumptions of the Grouping Model showing the default or bottom-up organizations in visual and motor buffers for the standard finger-cuing task (Miller, 1982) with adjacently placed hands.

As depicted in Figure 1.2, the Grouping Model presumes that cues and other visual stimuli are coded in a visual buffer while response codes are maintained in a motor buffer (Adam et al., 2003b). The buffers are supposed to interact. Thus, the results of stimulus coding can influence response processing and the results of response coding can affect stimulus processing. The key idea of the Grouping Model is that the individual elements of multielement visual displays and multi-element response arrays are not processed independently but are preattentively organized or "grouped" according to 
low-level grouping factors that may be both stimulus-driven (e.g., Gestalt principles) and response-related (e.g., the location and anatomical identity of responses).

The Grouping Model is based on two main assumptions, the grouping and the processing assumption, which are explained in the next two paragraphs.

\section{The grouping assumption}

According to the grouping assumption, the pattern of differential cuing benefits and the hand-advantage in particular are mostly a function of lowlevel grouping operations that specify stimulus and response subgroups. Consider Figure 1.2., which represents a four-element stimulus-response set. Note that the spatial arrangement strongly suggests a grouping of the two leftmost and two rightmost elements, both in the stimulus and response sets. As implied by the binary-tree structure, Adam et al. (2003b) assumed that such an implicit grouping scheme leads to a hierarchically clustered representation of the stimulus and response sets, with the two elements on either side forming a common cognitive representation on what one might call the "grouping level". In this scheme, hand-cues represent a unitary, well-defined "left" stimulus group that corresponds directly and unambiguously to an equally well-defined "left" response group. Thus, a "left" or "right" hand-cue directly activates the two relevant stimulus and response representations, thereby allowing swift and efficient selection (Adam et al., 2003b). In contrast, finger- and neither-cues that appear in any other pair of locations would activate elements belonging to two different groups (the left and the right group) in both the stimulus and the response set. Hence, response activation at the grouping level does not unambiguously identify the two cued elements as one group but requires a more effortful analysis of the information provided by the cue.

In sum, the grouping assumption states that the pattern of differential cuing benefits is mediated by interacting perceptual and motoric grouping factors. For that reason, cuing benefits may arise at multiple loci in the information processing system. Therefore, Adam et al. (2003b) examined the influence of manipulations in the stimulus set, in the response set, and in both sets. In their Experiment 1, they used three response sets: the standard index-middle fingers, an index-little fingers, and two one-hand response sets (left-hand and right-hand). The index-middle fingers response set, which suggested a grouping of responses in terms of left and right, demonstrated the expected hand-advantage, whereas the other response sets did not show a reliable 
hand-advantage. This was predicted by the Grouping Model because the index-little fingers response set was assumed to weaken the left-right distinction by strengthening the grouping of different hands (i.e., the two index fingers). Furthermore in the one-hand response set, the anatomical basis for the left-right response coding was removed, which made the "handadvantage" disappear. To conclude, Adam et al.'s Experiment 1 showed that the nature of the response-set is a strong determinant of the pattern of fingercuing effects, suggesting that grouping takes place and mediates performance in the motor buffer.

Experiments 2 and 3 investigated the joint influence of stimulus- and response-related grouping manipulations on the pattern of differential cuing benefits. In Experiment 2, Adam et al. (2003b) demonstrated such interactions by orthogonally manipulating the nature of the stimulus and response sets. Next to the standard stimulus and response sets with a clear left-right distinction - the stimulus-response set combination producing the typical left-right advantage - they also used stimulus and response sets with a clear inner-outer distinction. This latter organization was realized by grouping the two inner stimulus positions closer together than the two left-most and two right-most stimulus positions, and by using the index and little fingers from both hands. The orthogonal combination of these two stimulus sets and two response sets resulted in four different stimulus-response mappings: a leftright stimulus display combined with either a left-right (compatible) or an inner-outer response set (incompatible), and an inner-outer stimulus display combined with either a left-right (incompatible) or an inner-outer (compatible) response set. With the compatible stimulus-response arrangements the left-right grouping produced a left-right advantage, while the inner-outer grouping produced an advantage for the inner-outer cues. With the incompatible stimulus-response arrangements, the pattern of differential cuing benefits generally followed the grouping characteristics of the stimulus set, with the response set providing important constraints. That is, whereas the inner-outer response set allowed efficient implementation of the perceptually salient left-right distinction, the left-right response set did not allow easy implementation of the perceptually salient inner-outer distinction (Adam et al., 2003b). The Grouping Model explained this by assuming that the hand-distinction was also present in the inner-outer response set, thereby allowing left-right cues to be effective too. 
Experiment 3 was a replication of Experiment 2, but now with a response set, which did not contain the hand distinction. That is, in Experiment 3, the response set contained four fingers selected from one single hand-the right hand. The left-right distinction in this response set was implemented by using the first two digits (i.e., thumb and index finger) and the last two (i.e., ring and little fingers); the inner-outer distinction was implemented by using the first and last digit (i.e., thumb and ring finger) and the second and third (i.e., index and middle fingers). Thus, by using four fingers of one hand, Adam et al. (2003b) removed the dominant, hand-based left-right distinction, and, hence, reduced the relative strength of this grouping principle on the response side. Consequently, Experiment 3, relative to Experiment 2, showed that by eliminating the hand-distinction the superiority of the left-right cues disappeared. That is, with compatible stimulus-response sets (and with short preparation intervals) the left-right grouping produced a small, marginally significant left-right advantage, while the inner-outer groupings yielded a strong, robust inner-outer advantage (Adam et al., 2003b). Together, the outcomes of experiments 2 and 3 demonstrated that the interaction between stimulus and response set determined the pattern of differential cuing benefits and the hand-advantage.

Furthermore, in Experiment 5 Adam et al. (2003b) examined the overlapped hand placement manipulation (i.e., the index and middle fingers of both hands alternate on the response keys in the order: right index, left middle, right middle, left index). The usual advantage for the hand-cued (left-right) condition with the hands adjacent reversed to an advantage for the fingercued (inner-outer) condition with the hands overlapped. In other words, the advantage of preparing two fingers on one hand typically found with adjacent hand placement disappeared with the overlapped hand placement. This finding is inconsistent with the salient-features coding principle and previous studies by Reeve and Proctor (1984; Proctor \& Reeve, 1988) reporting that the hand-advantage is independent of the fingers used to respond. Instead, it supports the view that motoric factors play a major role in finger-cuing effects, a notion endorsed by the Grouping Model (Adam et al., 2003b, 2005).

\section{The automatic vs. effortful processing assumption}

According to the processing assumption of the Grouping Model, automatic and effortful processes mediate the pattern of differential cuing effects. This means that response preparation operates at multiple levels (cf. Jennings \& Van der Molen, 2005). 
The processing advantage of the hand-cued condition simply reflects the natural and stronger grouping of the two leftmost and two rightmost elements in both the stimulus display and the response array. In other words, each stimulus set and each response set has a default organization established preattentively by the bottom-up computation of perceptual and motoric units or subgroups. This process is fast and automatic (see Figure 1.3A). In contrast, finger- and neither-cues activate elements belonging to two different groups, the left and the right group, in both the stimulus and response dimensions (see Figure 1.3B).

With additional top-down processing, however, alternative group organizations can be attained. This process is slow and effortful. Thus, the pattern of cuing effects that emerges in the finger-cuing task critically depends on the nature of these default groupings and on the time available to reorganize these representations, if necessary. According to the Grouping Model, finger- and neither-cues are the more difficult cues because they require slow, effortful, top-down processing to break up the default, left-right spatial organization and to create a new organization based on the characteristics of the cue (see Figure 1.3B).

Adam et al. (2005) reported several lines of evidence that support the automatic vs. effortful processing assumption of the Grouping Model. First, when preparing two fingers on one hand, RT-benefits are visible during the first 100 ms of preparation, whereas finger- and neither-cues require longer preparation intervals (i.e., at least $250 \mathrm{~ms}$ ) to start producing preparation benefits. Second, older age does not affect the RT benefits associated with preparing two fingers on one hand, but does reduce preparation efficiency when preparing two fingers on two different hands (Adam et al., 1998). Assuming that advancing age is associated with a reduction in central resources, while leaving the automatic processes intact (Ford et al., 1997; Wishart et al., 2000), this finding is in accordance with the notion that handcues induce fast, automatic selection of subgroups, whereas finger-cues require slower, effortful processes to create a subgroup. Third, Adam et al. (1997) described a study with tactile stimuli. In contrast with the visual fingercuing task, where visual cues specify a subset of possible finger responses, the tactile cuing task specifies a subset of finger responses vibrotactually, and hence more directly. The study of Adam et al. (1997) showed that with vibrotactual cues the hand-advantage disappeared. This is in line with the Grouping Model because all cues directly and automatically specify the cued responses, thereby eliminating any possible differences in subgroup making. 


\section{A Subgroup Selection}

Hand-cued left

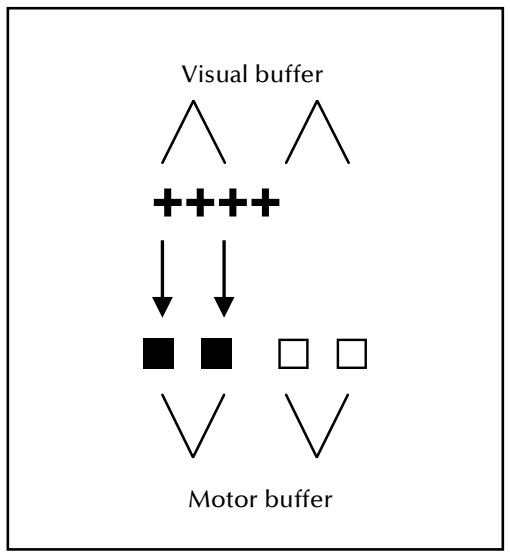

Hand-cued right

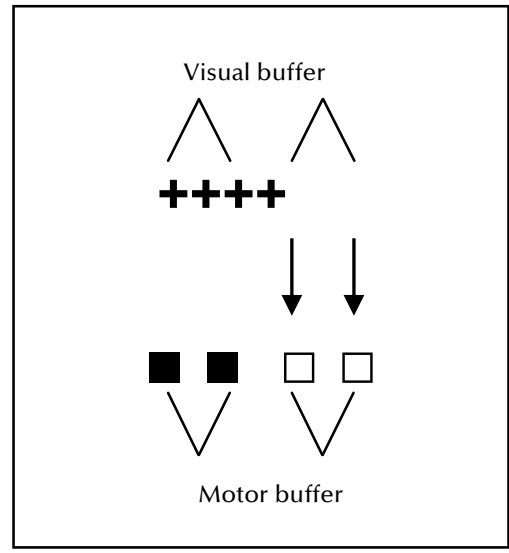

\section{B Subgroup Creation}

Finger-cued inner

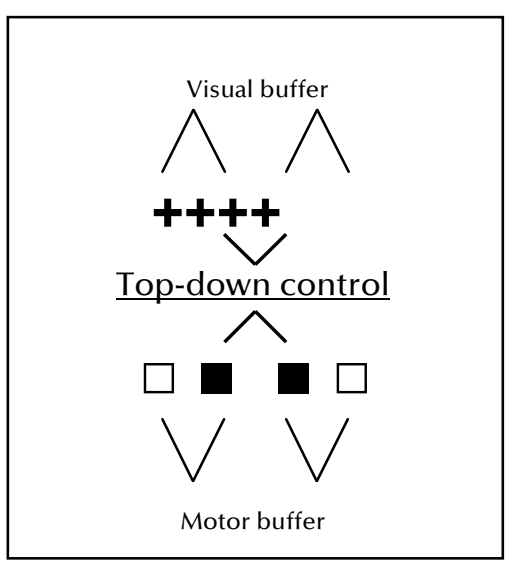

Finger-cued outer

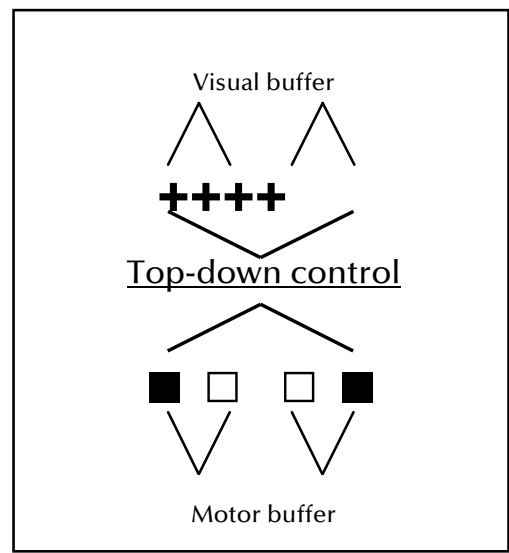

Figure 1.3. Sketch of the processing assumptions of the Grouping Model showing (A) subgroup selection or the bottom-up, exogenous control mode, versus (B) subgroup creation or the top-down, endogenous control mode. 
Finally, Adam et al. (2005) performed several experiments in which they varied the characteristics of the cue. In Experiment 1, onset (standard cuing procedure) and no-onset cues were used. In the no-onset condition, the cue initially consisted of four plus signs, after which two plus signs were removed. The remaining plus signs provided the valid cue information. The disappearing cue elements, however, automatically attract attention to their (previous) position, while the remaining elements do not. The crucial finding was that, with short preparation intervals of $100 \mathrm{~ms}$, cue type interacted with preparation condition. This interaction indicated that cue type (onset vs. noonset) modulated preparation efficiency of the hand-cued but not of the finger-cued. The finding that onset (but not no-onset) cues produce the handadvantage is consistent with the notion that onset hand-cues induce automatic, exogenous, bottom-up control, whereas no-onset finger-cues require slower, endogenous, top-down control. Adam et al.'s (2005) Experiment 2 showed that the usual hand-advantage present with peripheral, spatial cues disappears with a central, symbolic cue (letters indicating the different cue types) because symbolic cues require the slower, endogenous control mode. In Experiment 4, Adam et al. (2005) lowered cue probability to $50 \%$ so that the cues were no longer reliable with respect to the location of the upcoming stimulus. This manipulation was introduced to examine the ability of uninformative hand-cues and uninformative finger-cues to cause automatic response activation effects. According to the Grouping Model, hand-cues are especially effective when they can exploit the anatomical leftright (hand) distinction. Hence, a large effect of cue type (valid-invalid) was expected for hand-cues but not for finger-cues. Results showed that RTs of valid hand-cues were substantially shorter than those of invalid hand-cues. However, there was no effect of cue validity for finger-cues. Adam et al. (2005) concluded that hand-cues, but not finger-cues, generated automatic response activation effects. Together, these findings support the processing assumption of the Grouping Model that hand-cues exert a fast, automatic, bottom-up selection of the cued responses, whereas finger-cues require slower, effortful, top-down processes to create a selective preparatory set.

An fMRI study by Adam et al. (2003a) showed different activation patterns in the brain for hand-, finger-, and neither-cues. In particular, hand-cues were associated with enhanced levels of activity in the basal ganglia and reduced levels of activity in the parietal cortex relative to finger- and neither-cues. The less natural and more complex finger- and neither-cues that are positioned on both sides of the visual hemispace require more complex processing to create a subgroup, thereby producing more brain activity in the parietal 
cortex. In contrast, the visual cue indicating preparation of fingers on one hand represents a natural, strong (left or right) perceptual subgroup, which is established fast and automatically. The strong subcortical activity in the basal ganglia associated with the hand-cued condition might be interpreted in terms of a robust inhibition process generated by the basal ganglia and targeted at one hemisphere (that is, the hemisphere representing the two irrelevant responses). That is, Adam et al. (2003a) speculated that general inhibition of the (pre)motor cortex of one of the hemispheres is easier to implement than the more specific inhibition of two irrelevant responses represented in two hemispheres.

\section{PUPILLOMETRY}

In general, it is difficult to reveal the underlying processes of action preparation only on the basis of behavioral measures (Leuthold et al., 2004). For this reason, we collected both finger (i.e., RT) and pupillary responses. The cognitive pupillary response provides a continuous, independent measure of mental workload during effortful processing (Beatty \& LuceroWagoner, 2000) and may thus supplement RT measurements. Previous studies have shown that the pupillary system is sensitive to cognitive task manipulations (e.g., Beatty, 1982; Grandholm et al., 1996). Pupil dilation reflects variations in central processing load with a good precision and a high temporal resolution, although the pupillary response is slightly delayed $( \pm 500$ ms, depending on task and individual differences) (Just \& Carpenter, 1993; Beatty \& Lucero-Wagoner, 2000). More precisely, larger pupil dilation reflects greater processing load or mental effort (Andreassi, 2000; Beatty \& LuceroWagoner, 2000; Jennings \& Van der Molen, 2005). Before explaining this cognitive pupillary response in more detail, we briefly describe the basic anatomy of the pupil.

The pupil of the human eye is the opening in the center of the iris, which can dilate to about 8 to $9 \mathrm{~mm}$ in diameter and constrict to about $1.5 \mathrm{~mm}$. The pupil's basic physiological function is to adjust the amount of light that enters the eye in response to the current light intensity (light reflex). This is regulated by two sets of muscles (see Figure 1.4). On the one hand, there are the radial muscle fibers of the dilator pupillae, which increase the size of the pupil. These radial fibers are innervated by the sympathetic nervous system, which starts in the midbrain. On the other hand, there are the circular muscle fibers of the sphincter pupillae, which decrease the size of the pupil. The 
sphincter pupillae is innervated by the parasympathetic nervous system, which originates in the hypothalamus (Andreassi, 2000; Beatty \& LuceroWagoner, 2000; Stern \& Dunham, 1990).

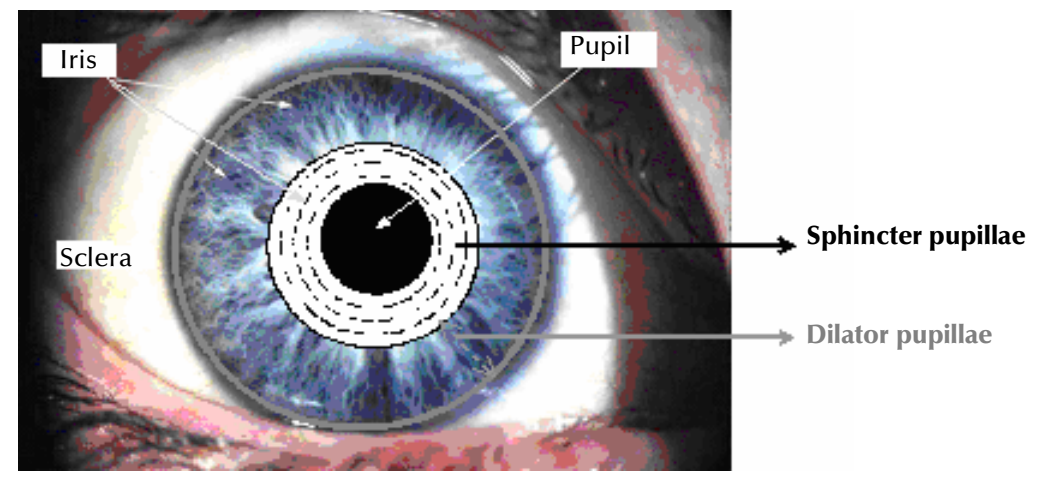

Figure 1.4. Diagram of the eye. Pupil constriction occurs as a result of contraction of the sphincter pupillae (circular muscle fibers), while pupil dilation results from contraction of the dilator pupillae (radial muscle fibers).

Task-evoked pupillary responses (TEPRs) are a reliable analytic tool to study processing load (Beattty \& Lucero-Wagoner, 2000). A baseline or tonic level is determined before trial onset or at a relevant pre-measurement interval. TEPRs represent the change in pupil size relative to this baseline level. Beatty and Lucero-Wagoner (2000) describe three useful TEPRs. First, mean pupil dilation during a post-stimulus time interval of interest. Second, peak dilation, which is the maximum dilation during this interval. Third, peak latency, which reflects the time elapsed between stimulus onset and peak dilation. It is important to note that mean pupil dilation, which is based on the average of many data points, is the most reliable TEPR, and therefore a primary dependent measure in this thesis.

TEPRs have shown to be sensitive to task manipulations in many studies of perception, memory, aging, information processing, arousal, and language, which all report larger mean pupil dilations with increasing task difficulty (e.g., Beatty, 1982; Beatty \& Lucero-Wagoner, 2000; Granholm et al., 1996). Recently a new boost of research has consolidated the broad use of TEPRs in studies of attention, memory, perceptual stability, language, arousal/ attractiveness, sexual interest, decision making, and deception in experiments and games (e.g., poker) (Demos et al., 2008; Einhauser et al., 2008; Heitz et al., 2008; Kuchinke et al., 2007). Furthermore, pupillometry abnormalities might even be used as early indicators of persons who are at high risk of future 
microvascular disease, depression and Autism Spectrum Disorder (Anderson et al., 2008; Bishop, 2006; Laeng \& Falkenberg, 2007; Maguire et al., 2008; Silk, 2007). In addition, pupil size changes might be a fast, non-invasive and efficient additional marker in the diagnosis of Alzheimer's disease (Fotiou et al., 2007). The clinical relevance of pupil responses was also found in earlier studies on aging, sleepiness and schizophrenia (Andreassi, 2000, Merritt et al., 2004; Minassian et al., 2004).

Furthermore, pupillometry has proved its excellence to measure response preparation. Previous response preparation studies typically used a simple RT paradigm (i.e., one target with one pre-specified response) and focused on the effects of stimulus uncertainty (Bitsios et al., 2004; Bradshaw, 1968; Jennings et al., 1998; Richer et al., 1983). In the current thesis, however, we focused on selective preparation of a subset of possible responses (i.e., event uncertainty) and used a four- instead of a one- or two-choice RT task.

\section{AIMS AND OUTLINE OF THE THESIS}

All experiments in the present thesis were aimed at testing the two main assumptions of the Grouping Model of finger preparation (Adam et al., 2003b, 2005): the grouping and the processing assumption. The grouping assumption stresses the importance of low-level grouping factors that specify stimulus and response groups. In this view, hand-cues are particularly effective cues because they indicate both a strong perceptual and a corresponding strong response group (a left and a right group). Consequently, hand-cues prompt a fast, automatic, bottom-up selection of the cued responses. In contrast, cues that indicate stimuli belonging to less defined or different groups (i.e., fingerand neither-cues) require a slower, effortful, top-down process to select the relevant responses, as captured by the automatic vs. effortful processing assumption. In all studies, reaction time (RT) and response accuracy (percentage errors) were calculated. In addition, we measured pupil dilation as an independent, continuous measure of cognitive workload during response preparation. In general, more difficult, effortful preparatory processes were expected to lead to larger pupil dilation than easier, more automatic preparatory processes.

In our first study, reported in Chapter 2, we examined if the pattern of differential preparation benefits is reflected by pupil dilation. Based on the processing assumption of the Grouping Model, it was expected that the 
selection and preparation of more difficult response sets (i.e., two fingers on two hands) is more effortful and therefore associated with larger pupil dilations than preparing easier response sets (i.e., two fingers on one hand).

In Chapter 3, we varied the response set to test the grouping assumption. More specifically, we disturbed the compatibility between the stimulus and response set by using an overlapped hand placement next to an adjacent hand placement. According to the grouping assumption, overlapping the hands largely reduces the good grouping of the two rightmost and leftmost response elements in the response set (loss of the anatomical left-right distinction). On that account, overlapped hands were expected to increase the processing load of hand-cues, thereby decreasing or eliminating the pattern of differential processing load, which is normally found with the conventional adjacent hand placement.

In Chapter 4, we manipulated cue validity $(75 \%$ valid cues and $25 \%$ invalid cues) to further test the processing assumption. Invalid cues incorrectly specify fingers and therefore require reprogramming operations to prepare the correct finger response. Because reprogramming requires time and effort, invalid cues are hypothesized to increase RT and pupil dilation. In particular, invalid cues are expected to reverse the normal pattern of pupil dilation as a function of the differential cuing benefits, because reprogramming "automatic" invalid cues (i.e., hand-cues) requires more effort than reprogramming the "more controlled" invalid cues (i.e., finger- and neithercues).

In Chapter 5, we investigated the potential role of inhibititory processes in the preparation of finger responses by using a moving hands response set next to the standard static hands response set. As proposed by Adam et al. (2003b), the hand-advantage of preparing two fingers on one hand (represented in one hemisphere) as opposed to preparing two fingers on two hands (represented in two hemispheres) is partly due to a response inhibition process that runs more efficiently within than between hemispheres. With the moving hands manipulation, we intended to decrease the within-hemisphere inhibition advantage relative to static hands, because moving the hands activates both hemispheres, thereby reducing the influence of inhibitory processes (Miller \& Adam, 2006). According to the response inhibition account, moving the hands should only influence the easier, bottom-up processing of the hand-cued (by decreasing the hand-advantage) while leaving the more effortful, top-down processes unaffected. 
In Chapter 6, we provided a last test of the processing assumption by examining whether there is an age-related deficit in the preparation of finger responses. It is known that aging affects effortful, but not automatic processing (e.g., Ford et al., 1997; Wishart et al., 2000). Hence, it is expected that advancing age will affect the preparation of hand-cues more than the preparation of finger- and neither-cues. Increasing the available time for preparation, however, may wash out this differential aging effect.

In Chapter 7, finally, the main findings reported in this thesis are summarized and discussed. In addition, recommendations for future research are provided.

\section{REFERENCES}

Adam, J., Keyson, D.V., Paas, F.G.W.C. (1997). Vibro tactual choice reaction time in a precuing paradigm. Human Movement Science, 16, 549-565.

Adam, J. J. (1994). Manipulating the spatial arrangement of stimuli in a precuing task. Acta Psychologica (Amst), 85(3), 183-202.

Adam, J. J., Backes, W., Rijcken, J., Hofman, P., Kuipers, H., \& Jolles, J. (2003a). Rapid visuomotor preparation in the human brain: a functional MRI study. Brain Research: Cognitive Brain Research, 16(1), 1-10.

Adam, J. J., Hommel, B., \& Umilta, C. (2003b). Preparing for perception and action (I): the role of grouping in the response-cuing paradigm. Cognitive Psychology, 46(3), 302-358.

Adam, J. J., Hommel, B., \& Umiltà, C. (2005). Preparing for perception and action (II) Automatic and effortful Processes in Response cuing. Visual Cognition, 12(8), 1444-1473.

Adam, J. J., Paas, F. G., Teeken, J. C., van Loon, E. M., van Boxtel, M. P., Houx, P. J., et al. (1998). Effects of age on performance in a finger-precuing task. Journal of Experimental Psychology: Human Perception and Performance, 24(3), 870-883.

Adam, J. J., \& Pratt, J. (2004). Dissociating visual attention and effector selection in spatial precuing tasks. Journal of Experimental Psychology: Human Perception and Performance, 30(6), 10921106.

Anderson, C. J., Colombo, J., \& Jill Shaddy, D. (2006). Visual scanning and pupillary responses in young children with Autism Spectrum Disorder. Journal of Clinical Experimental Neuropsychology, 28(7), 1238-1256.

Andreassi, J. L. (2000). Pupillary response and behaviour. In N.J. Mahwah (Ed.), Psychophysiology. Human behaviour and Physiological response (4th ed., Vol. 10, pp. 218-233). New York: Oxford University Press.

Beatty, J. (1982). Task-evoked pupillary responses, processing load, and the structure of processing resources. Psychological Bulletin, 91(2), 276-292.

Beatty, J., \& Lucero-Wagoner, B. L. (2000). The Pupillary system. In J. T. Cacioppo, L. G. Tassinary \& G. B. Berntson (Eds.), Handbook of Psychophysiology (2nd ed., Vol. 2nd edition, pp. 142-162). United States of America: Cambridge University Press.

Bishop, M. (2006). Pupil dilation could be a depression-risk biomarker for adolescents taking therapeutic steriods: Presented at AACAP. Human Factors, 48(4), 651-655. 
Bitsios, P., Szabadi, E., \& Bradshaw, C. M. (2004). The fear-inhibited light reflex: importance of the anticipation of an aversive event. International Journal of Psychophysiology, 52(1), 87-95.

Bradshaw, J. L. (1968). Pupillary changes and reaction time with varied stimulus uncertainty. Psychonomic Science, 13(2), 69-70.

de Jong, R., Wierda, M., Mulder, G., \& Mulder, L. J. (1988). Use of partial stimulus information in response processing. Journal of Experimental Psychology: Human Perception and Performance, 14(4), 682-692.

Demos, K. E., Kelley, W. M., Ryan, S. L., Davis, F. C., \& Whalen, P. J. (2008). Human Amygdala Sensitivity to the Pupil Size of Others. Cerebral Cortex. 18, 2729-2734

Einhauser, W., Stout, J., Koch, C., \& Carter, O. (2008). Pupil dilation reflects perceptual selection and predicts subsequent stability in perceptual rivalry. Proceedings of the National Academy of Sciences USA, 105(5), 1704-1709.

Ford, J. M., Roth, W. T., Isaacks, B. G., Tinklenberg, J. R., Yesavage, J., \& Pfefferbaum, A. (1997). Automatic and effortful processing in aging and dementia: event-related brain potentials. Neurobiology of Aging, 18(2), 169-180.

Fotiou, D. F., Brozou, C. G., Haidich, A. B., Tsiptsios, D., Nakou, M., Kabitsi, A., et al. (2007). Pupil reaction to light in Alzheimer's disease: evaluation of pupil size changes and mobility. Aging Clinical Experimental Research, 19, 364-371.

Granholm, E., Asarnow, R. F., Sarkin, A. J., \& Dykes, K. L. (1996). Pupillary responses index cognitive resource limitations. Psychophysiology, 33(4), 457-461.

Heitz, R. P., Schrock, J. C., Payne, T. W., \& Engle, R. W. (2008). Effects of incentive on working memory capacity: behavioral and pupillometric data. Psychophysiology, 45(1), 119-129.

Hick, W. E. (1952). On the rate of gain of information. Quarterly Journal of Experimental Psychology, 4, 11-26.

Hyman, R. (1953). Stimulus information as a determinant of reaction time. Journal of Experimental Psychology, 45(3), 188-196.

Jennings, J. R., \& van der Molen, M. W. (2005). Preparation for speeded action as a psychophysiological concept. Psychological Bulletin, 131(3), 434-459.

Jennings, J. R., van der Molen, M. W., \& Steinhauer, S. R. (1998). Preparing the heart, eye, and brain: foreperiod length effects in a nonaging paradigm. Psychophysiology, 35(1), 90-98.

Just, M. A., Carpenter, P.A., Miyake, A. (2003). Neuroindices of cognitive workload: neuroimaging, pupillometric and event-related potential studies of brain work. Theoretical Issues in Ergonomic Science, 4(NOS 1-2), 56-88.

Kuchinke, L., Vo, M. L., Hofmann, M., \& Jacobs, A. M. (2007). Pupillary responses during lexical decisions vary with word frequency but not emotional valence. International Journal of Psychophysiology, 65(2), 132-140.

Laeng, B., \& Falkenberg, L. (2007). Women's pupillary responses to sexually significant others during the hormonal cycle. Hormones and Behavior, 52(4), 520-530.

Leuthold, H., Sommer, W., \& Ulrich, R. (1996). Partial advance information and response preparation: inferences from the lateralized readiness potential. Journal of Experimental Psychology General, 125(3), 307-323.

Leuthold, H., Sommer, W., \& Ulrich, R. (2004). Preparing for Action: Inferences from CNV and LRP. Journal of psychophysiology, 18, 77-88.

Maguire, A. M., Craig, M. E., Craighead, A., Chan, A. K., Cusumano, J. M., Hing, S. J., et al. (2007). Autonomic nerve testing predicts the development of complications: a 12-year follow-up study. Diabetes Care, 30(1), 77-82.

Merritt, S. L., Schnyders, H. C., Patel, M., Basner, R. C., \& O'Neill, W. (2004). Pupil staging and EEG measurement of sleepiness. International Journal of Psychophysiology, 52(1), 97-112. 
Miller, J. (1982). Discrete versus continuous stage models of human information processing: in search of partial output. Journal of Experimental Psychology Human Perception and Performance, 8(2), 273-296.

Miller, J. (1985). A hand advantage in preparation of simple keypress responses: reply to Reeve and Proctor. Journal of experimental psychology, 11(2), 221-233.

Miller, J. (1988). Discrete and continuous models of human information processing: theoretical distinctions and empirical results. Acta Psychologica (Amst), 67(3), 191-257.

Miller, J., \& Adam, J. J. (2006). Redundancy gain with static versus moving hands: a test of the hemispheric coactivation model. Acta Psychologica (Amst), 122(1), 1-10.

Minassian, A., Granholm, E., Verney, S., \& Perry, W. (2004). Pupillary dilation to simple vs. complex tasks and its relationship to thought disturbance in schizophrenia patients. International Journal of Psychophysiology, 52(1), 53-62.

Proctor, R. W., \& Reeve, T. G. (1986). Salient-Feature Coding Operations in Spatial Precuing Tasks. Journal of Experimental Psychology: Human Perception and Performance., 12(3), 277-285.

Proctor, R. W., \& Reeve, T. G. (1988). The acquisition of task-specific productions and modifications of declarative representations in spatial-precuing tasks. Journal of Experimental Psychology General, 117(2), 182-196.

Proctor, R. W., Reeve, T. G., \& van Zandt, T. (1992). Salient-feature coding in response selection. In G. E. Stelmach \& J. Requin (Eds.), Tutorials in motor behavior II. Amsterdam: North Holland.

Proctor, R. W., Vu, K. P., \& Pick, D. F. (2006). A deficit in older adults' effortful selection of cued responses. Journal of Motor Behavior, 38(4), 265-284.

Reeve, T. G., \& Proctor, R. W. (1984). On the advance preparation of discrete finger responses. Journal of Experimental Psychology Human Perception and Performance, 10(4), 541-553.

Reeve, T. G., \& Proctor, R. W. (1990). The salient-features coding principle for spatial-and symboliccompatibility effects. In R. W. Proctor \& T. G. Reeve (Eds.), Stimulus-response compatibility (pp. 163-180). Amsterdam: North-Holland.

Richer, F., Silverman, C., \& Beatty, J. (1983). Response selection and initiation in speeded reactions: a pupillometric analysis. Journal of Experimental Psychology Human Perception and Performance, 9(3), 360-370.

Rosenbaum, D. A. (1980). Human movement initiation: specification of arm, direction, and extent. Journal of Experimental Psychology General, 109(4), 444-474.

Silk, J. S., Dahl, R. E., Ryan, N. D., Forbes, E. E., Axelson, D. A., Birmaher, B., et al. (2007). Pupillary reactivity to emotional information in child and adolescent depression: links to clinical and ecological measures. The American Journal of Psychiatry, 164(12), 1873-1880.

Stern, J. A., \& Dunham, D.N. (1990). The ocular system. In J. T. Cacioppo, Tassinary, L.G. (Ed.), Principles of psychophysiology (pp. 193-215). Cambridge, England: Cambridge University press.

Van Gerven, P. W. M., Paas, F., Van Merriënboer, J. J. G., \& Schmidt, H. G. (2004). Memory load and the cognitive pupillary response in aging. Psychophysiology, 41, 167-174.

Wishart, L. R., Lee, T. D., Murdoch, J. E., \& Hodges, N. J. (2000). Effects of aging on automatic and effortful processes in bimanual coordination. Journal of Gerontology Series B Biological, Psychological Sciences and Social Sciences, 55(2), P85-94. 


\section{PUPIL DILATION IN RESPONSE PREPARATION}

\section{CHAPTER 2}

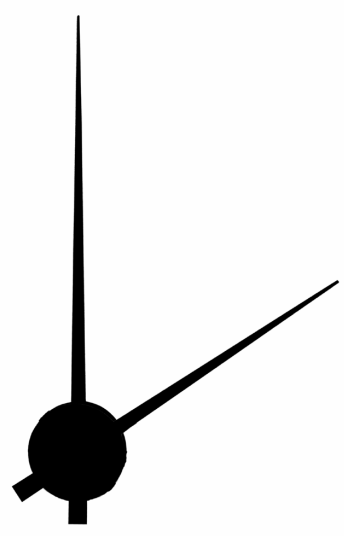

Sofie Moresi, Jos J. Adam, Jons Rijcken,

Pascal W.M. Van Gerven, Harm Kuipers \& Jelle Jolles (2008).

Pupil Dilation in Response Preparation.

International Journal of Psychophysiology,

$67(2)$, 124-130. 


\section{ABSTRACT}

This study examined changes in pupil size during response preparation in a finger-cuing task. Based on the Grouping Model of finger preparation (Adam et al., 2003b; 2005), it was hypothesized that the selection and preparation of more difficult response sets would be accompanied by larger pupillary dilations. The results supported this prediction, thereby extending the validity of pupil size as a measure of cognitive load to the domain of response preparation.

\section{INTRODUCTION}

Preparing to act facilitates motor performance. The underlying processes of this performance enhancement have been studied by means of responsecuing paradigms, in which cues provide information about some, or all, of the required response parameters before the actual target stimulus appears. Thus, cues in response-cuing paradigms allow and induce a process of response preparation that facilitates motor performance. For instance, in the finger-cuing paradigm developed by Miller (1982), a visual cue signal indicates a subset of two possible finger (i.e., key press) responses out of a total of four, thus allowing the selection and preparation of a subset of finger responses. The robust finding from this paradigm is that, given sufficient preparation time, informative cues substantially shorten reaction time (RT) relative to an uncued condition (Adam, Hommel, \& Umiltà, 2003b; Reeve \& Proctor, 1990). The present study extends the numerous chronometric studies on response preparation by examining its effect on a psychophysiological measure, pupil dilation, with the purpose of further delineating its underlying mechanisms.

The cognitive pupillary response or the task-related change in pupil size has been shown to be a reliable measure of processing load and resource allocation, with larger pupil dilation reflecting greater processing load or mental effort. This has been established in many studies of language processing, perception, memory, complex reasoning, and attention, which all reported larger pupil dilations for more difficult tasks (Andreassi, 2000; Beatty \& Lucero-Wagoner, 2000; Jennings \& Van der Molen, 2005). In the present study, we used the sensitivity of the pupillary response to task complexity to test a theoretical account (Adam, Hommel, \& Umiltà, 2003b, 2005) of an interesting phenomenon typically observed in the finger-cuing task, namely a 
pattern of differential preparation benefits. Before explaining this phenomenon and the proposed account, we first describe the finger-cuing task in detail.

In the finger-cuing task (Miller, 1982), participants are forewarned by a visual cue signal about a particular subset of possible finger responses. Typically, they respond to horizontally arranged stimuli by spatially compatible key press responses with the index and middle fingers of both hands placed adjacently. In the present study, the visual display consists of four white boxes on a computer monitor, in which the cue and target signals were presented (see Figure 2.1). At the start of a trial, a neutral warning stimulus ("+" sign) appeared between the two center boxes for $1 \mathrm{~s}$. Then, the cue signal was presented for $2 \mathrm{~s}$ by coloring two boxes grey. Following the cue signal, the target stimulus was presented by making one of the two grey boxes black, thus indicating the required finger response. The 2-s time interval between onset of the cue and onset of the target stimulus is called the preparation interval, as it reflects the amount of time participants have to selectively prepare the two finger responses indicated by the cue before the appearance of the target. The functional significance of the cue is that it transforms the basic four-choice task into a two-choice task. Four cue or preparation conditions can be distinguished (Figure 1).

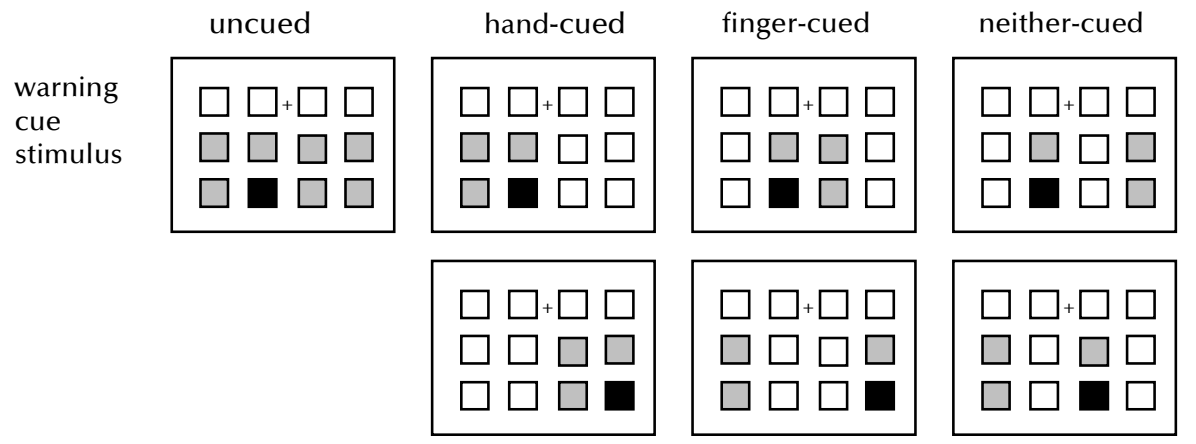

Figure 2.1. A schematic representation of the finger-cuing task. Cue and target stimuli are presented overlaid and not in separate rows.

In the hand-cued condition, the cue specifies two fingers on the same hand (e.g., the left-middle and left-index fingers). In the finger-cued condition, the cue specifies the same finger on two hands (e.g., the left-index and rightindex fingers). In the neither-cued condition, the cue specifies different 
fingers on two hands (e.g., the left-index and right-middle fingers). These three preparation conditions are called the "cued" conditions. Also, an uncued control condition is included. Here, the cue does not provide advance information about the upcoming response (all four boxes turn grey), thus precluding selective response preparation. In other words, the uncued condition leaves the basic 4-choice task unaltered and, thus, is a control or baseline condition, against which the effects of the "cued" conditions can be evaluated. Since RTs in a 2-choice task are substantially shorter than RTs in a 4-choice task (Hick, 1952; Hyman, 1953), cue effectiveness is inferred from a significant RT advantage or benefit for the 2-choice "cued" conditions (i.e., hand-cued, finger-cued, and neither-cued) over the control, 4-choice (uncued) condition. Thus, with longer preparation intervals $(1000 \mathrm{~ms}$ and more), hand-cued, finger-cued, and neither-cued conditions all show substantially shorter RTs than the uncued condition, reflecting the operation of selective preparation.

A strong and often replicated observation in the finger-cuing paradigm is a pattern of differential cuing benefits: RTs are shortest for the hand-cued condition, longest for the neither-cued condition, and intermediate for the finger-cued condition, suggesting an ordering in terms of preparation difficulty (Adam, Hommel, \& Umiltà, 2003b; Reeve \& Proctor, 1990). It should be noted, however, that this pattern of differential cuing benefits only emerges with short preparation intervals (i.e., intervals less than about $1.5 \mathrm{~s}$ ). When the preparation interval is extended to $2 \mathrm{~s}$ or more, the three cued conditions often show comparable RTs. Thus, certain pairs of responses can be selected and prepared more quickly than others, with small or no differences between the pairs given sufficiently long preparation time.

A recent account of the pattern of differential cuing benefits is the Grouping Model (Adam, Hommel, \& Umiltà, 2003b, 2005), which is an extension of the salient-features coding principle (T.G. Reeve \& Proctor, 1990). The key idea of the Grouping Model is that the individual elements of multi-element visual displays and multi-element response arrays are not processed independently but are preattentively organized or "grouped" according to low-level grouping factors that may depend on stimulus-driven (e.g., Gestalt principles) and response-related factors (e.g., inter-response dependencies). Thus, when presented with an array of four horizontally aligned potential target locations (centered on a person's midline), left-right (i.e., hand-) cues are easily encoded into left or right visual groups based on the Gestalt principle of proximity. In addition, such left-right cues are also easily encoded into left- 
right response groups which involve the two fingers on the left or right hands. No other set of cues affords such simple, strong perceptual-motor subgroups, as both inner-outer (i.e., finger-) cues and alternate (i.e., neither) cues require binding separate items across the midline. The outcome of this is that left-right or hand-cues can activate responses in a fast, bottom-up manner while the other cues must activate responses in a slower, top-down manner. Left-right cues may also cause automatic shifts of attention to the cued locations while bilateral cues may require volitional shifts of attention (Adam et al., 2003, 2005). Together, these perceptual, motor, and attentional factors produce the left-right or hand-cued advantage.

There are now several experiments that support the Grouping Model. For example, the advantage of left-right or hand-cues can be reduced, and even eliminated, if the distance between the two inner cues is greatly reduced (i.e., making the inner cues the easiest to encode) (e.g., Adam et al., 2003, Experiment 2; Reeve, et al., 1992, Experiment 2) or if the four responses are mapped onto a single hand (i.e., eliminating the response grouping present with two fingers on two hands) (e.g., Adam et al., 2003; Proctor \& Reeve, 1986). Additionally, no-onset cues do not yield a hand-cued advantage, as the lack of an abrupt onset does not capture attention at the (left-right) cued locations (Adam et al., 2005, Experiment 1).

In sum, according to the Grouping Model, the processing advantage of the hand-cued condition simply reflects the natural and stronger grouping of the two leftmost and two rightmost elements in both the stimulus and response sets. That is, each stimulus set and each response set has a default organization established preattentively by the bottom-up computation of perceptual and motor units or subgroups. This process is fast and automatic. With additional, top-down processing, however, alternative organizations can be attained. This process is slow and effortful. Thus, the pattern of precuing effects that emerges in the finger-cuing task critically depends on the nature of these default groupings and on the time available to reorganize these representations, if necessary.

According to the Grouping Model, finger- and neither-cues are the more difficult cues because they require slow, effortful, top-down processing to break up the default, left-right spatial organization and to create a new perceptual and motor organization based on the characteristics of the cue. Furthermore, in this view, the neither-cued condition is more difficult than the finger-cued condition, because the former represents a perceptually asymmetrical subgroup that indicates the selection and preparation of two 
different, non-homologous fingers on two hands, whereas the latter represents a perceptually symmetrical subgroup that specifies the same or homologous fingers on two hands. Because homologous fingers are neurally and functionally linked (Rosenbaum, 1991), they are easier to group than nonhomologous fingers.

Assumptions about good grouping are corroborated by independent evidence. For instance, the strong grouping of left and right perceptual subgroups is bolstered by research showing that people spontaneously and naturally divide the visual space into left-side and right-side parts (e.g., Mapp \& Ono, 1999; Nicoletti \& Umiltà, 1989). The stronger grouping of fingers on one hand as opposed to fingers on different hands is substantiated by the fact that cerebral control of hand and finger movements is almost completely localized in the contralateral frontal lobe (e.g., Hellige, 1993).

Additional support for the Grouping Model comes from an aging study, which showed that older age does not affect the RT benefits associated with hand-cues, but does strongly reduce the efficiency of the finger- and neithercues, at least with preparation intervals equal or shorter than $2 \mathrm{~s}$ (Adam et al., 1998). Given that advancing age is accompanied by a reduction in central resources (Allen, Groth, Weber, \& Madden, 1993), while leaving automatic processes intact (Hasher \& Zacks, 1979), this finding accords with the idea that hand-cues prompt the fast, bottom-up selection and preparation of fingers, whereas the more difficult finger- and neither-cues require slow and effortful top-down processes to establish a selective preparatory set.

The aim of the present study was to examine the effects of different response cues in the finger-cuing task on pupil dilation to provide a further test of the Grouping Model. The logic of the study was straightforward: if the pattern of differential cuing benefits in the finger-cuing task is associated with differences in processing load or mental effort, with more difficult cues requiring more effortful processing, the largest pupillary dilation should be observed in the neither-cued condition, the smallest in the hand-cued condition, and an intermediate level of pupillary dilation in the finger-cued condition. Because we used a rather long preparation interval of $2 \mathrm{~s}$ (to allow sufficient time for the pupil to react), we expected all informative cue conditions (hand-, finger-, and neither-cues) to generate substantial RT benefits relative to the uncued condition. Importantly, however, the underlying preparatory processes that generated these benefits are expected 
to be different in terms of the involved processing load, and thus to be reflected in the pupillary response.

\section{METHOD}

\section{Participants}

The experiment was carried out with 18 right-handed health-science, medicine, and psychology students from Maastricht University (9 women, 9 men; mean age $=22.7$ years, $\mathrm{SD}=2.52$ ). All had normal or corrected-to-normal vision. None of them took eye medication that could influence pupil responses. Informed consent was obtained and they received $€ 7.50$ for their participation.

\section{Stimuli and apparatus}

The visual display consisted of a row of four, white boxes $(1 \times 1 \mathrm{~cm})$ in black outline, presented on a standard computer screen with a grey background, which corresponded to the calibration background. All four boxes were separated by $0.5 \mathrm{~cm}$. Subjects were seated in a height-adjustable chair with their heads stabilized in a chinrest placed at a distance of $57 \mathrm{~cm}$ from the monitor. Subjects placed the index and middle fingers of both hands on four linearly arrayed push buttons mounted on a response box, placed in front of the subjects on a table, and aligned with the centre of the stimulus set. The illuminance in the room was constant (about $600 \mathrm{~lx}$ ). RTs were measured in milliseconds (ms). The software controlling the RT measurements and the stimulus presentation was programmed in Matlab 7 and supported by the Eyelink Toolbox (Brainard, 1997; Cornelissen, Peters, \& Palmer, 2002). The pupil area was measured in pixels by the Eyelink I Gazetracker (SR Research Ltd., Canada), a head-mounted infrared video-based tracking system with a temporal resolution of $250 \mathrm{~Hz}$ and a spatial resolution of at least $0.01^{\circ}$.

\section{Design and procedure}

Each subject received a series of 80 test trials, preceded by 16 practice trials. Within the series of 80 test trials there were 20 trials for each cue condition (uncued, hand-cued, finger-cued, neither-cued) in a random order. Subjects were informed about the nature of the task and were explicitly told to take advantage of the informative cues. They were instructed to react as quickly as possible to the target stimulus by pressing the correct, spatially corresponding, response key. No feedback was provided. At the start of each 
individual test session, a nine-point calibration and validation procedure was performed.

\section{Analysis}

Trials with RTs of less than $125 \mathrm{~ms}$ or in excess of $1250 \mathrm{~ms}$ (1.46\%) were considered outliers and were excluded from all data analyses. Mean RT and proportion of errors were calculated for each subject as a function of cue condition. Pupil data from the right eye were analyzed and only for correct trials (i.e., trials without response errors and RT outliers). Eye blinks were filtered out by a computer algorithm and area samples were replaced by cubic spline interpolation, starting 17 samples (i.e., $68 \mathrm{~ms}$ ) before and ending 25 samples (i.e., $100 \mathrm{~ms}$ ) after the blink. For each trial, a baseline pupil area was determined by calculating the average pupil size during a period of $100 \mathrm{~ms}$ preceding the onset of the cue signal. Cue-locked pupil dilation was calculated by subtracting this baseline area from the pupil area for each data point during a period of $4 \mathrm{~s}$ following cue onset. This difference score was converted to a percentage of the corresponding baseline value. This conversion was warranted by Jin's (1992) revised version of the so-called Law of Initial Value (LIV), which states that within the middle range of baseline values, the physiological response increases as a function of baseline. This version of the LIV was applicable to our data, because there was a positive overall correlation between baseline pupil size and peak dilation $(r=.53)$. The LIV introduces a physiological artifact, however, because it influences pupil dilation and thus may obscure the effect of cue condition. Expressing dilation as a percentage of the baseline corrects for this artifact, because pupillary responses following a relatively high baseline value are attenuated. This procedure was earlier applied by, for example, Van Gerven et al. (2004).

\section{RESULTS}

\section{Behavioral data}

As expected, RTs in the three cued conditions were all substantially shorter $(\mathrm{M}=65 \mathrm{~ms})$ than those in the uncued condition $(\mathrm{Ms}=340 \mathrm{~ms}, 325 \mathrm{~ms}, 335 \mathrm{~ms}$, and $398 \mathrm{~ms}$, for hand-cued, finger-cued, neither-cued, and uncued conditions, respectively). A one-way repeated-measures analysis of variance (ANOVA) on the RT data confirmed this picture by revealing a highly significant effect of cue condition, $F(3,51)=58.12, \eta 2=.77, p<.001$. Tukey Least Significant Difference (LSD) post-hoc tests revealed large significant benefits for all three cued conditions relative to the uncued condition ( $p s<.001$ ), indicating that 
participants selectively prepared responses in all three cued conditions. Furthermore, the post-hoc tests revealed a small (i.e., $15 \mathrm{~ms}$ ) but significant ( $p$ $<$.05) RT advantage for the finger-cued condition over the hand-cued condition. All other comparisons between the three cued conditions were not significant. Subjects made few errors, with a mean error rate of $1.5 \%(\mathrm{Ms}=$ $0.4 \%, 0.2 \%, 1.9 \%$, and $3.8 \%$ for hand-cued, finger-cued, neither-cued, and uncued conditions, respectively). This number was too low for statistical analysis. Note, however, that the error rates mirror the RT time pattern, with fewer errors for the cued conditions than for the uncued condition, indicating that the cuing benefits were not due to a speed-accuracy trade-off.

\section{Pupil data}

Figure 2.2 shows the mean pupil dilation (averaged over subjects) as a function of time (starting at cue onset until $3.5 \mathrm{~s}$ after cue onset) and cue condition (uncued, hand-cued, finger-cued, and neither-cued). This dilationby-time plot reveals that the pupil started to dilate about $350 \mathrm{~ms}$ after cue onset, with thereafter a clear differentiation in the development of the dilation curves for the three cued conditions. Whereas the neither-cued condition showed a steady and continuous increase in pupil size throughout the preparation interval, the hand-cued condition tended to show a slight decrease midway through the preparation interval and a steady increase thereafter. The finger-cued condition showed an intermediate pattern, with a period of constant pupil size about halfway the preparation interval. 


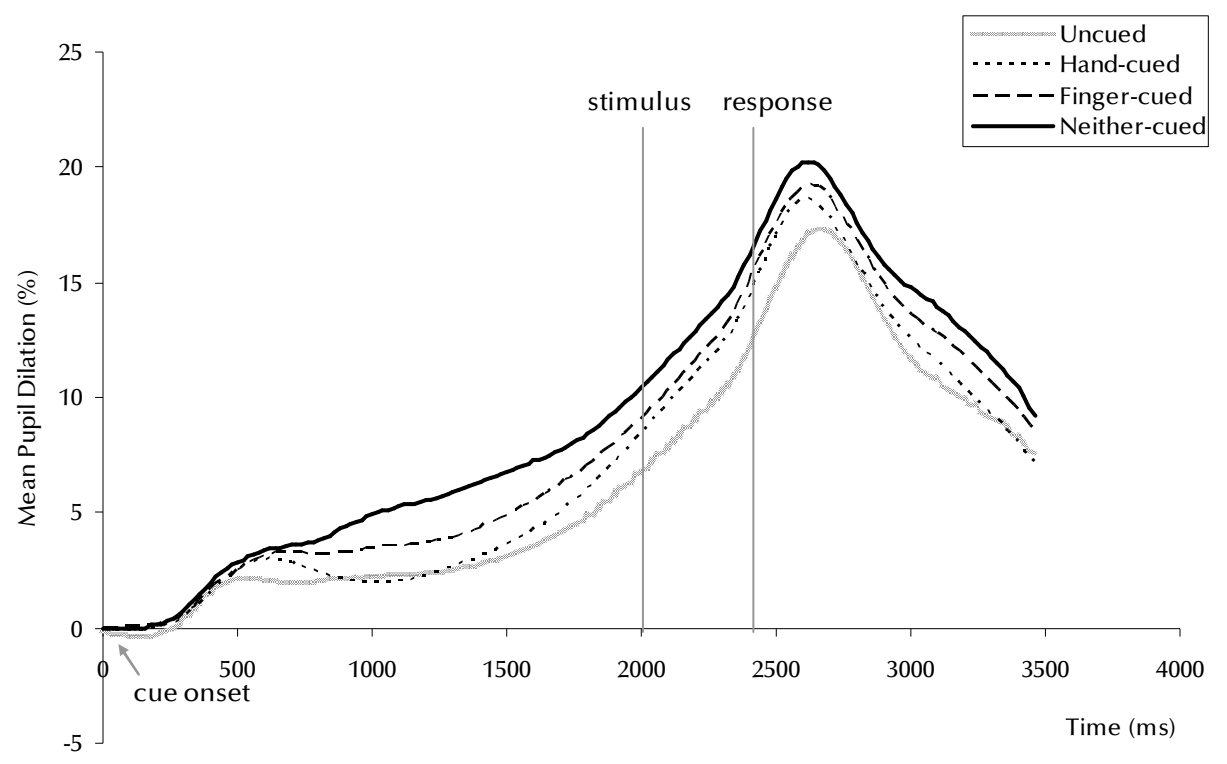

Figure 2.2. A dilation-by-time plot for the four cue conditions starting from cue onset until $3.5 \mathrm{~s}$ afterwards. The stimulus is presented $2 \mathrm{~s}$ after cue onset and the response occurs on average $350 \mathrm{~ms}$ after stimulus onset.

From Figure 2.2 it is clear that pupil dilation during the preparation interval is directly related to cue difficulty, with the neither-cued condition producing the largest pupil dilation, the hand-cued condition the smallest, and the finger-cued condition an intermediate dilation. A one-way repeated-measures ANOVA on the mean increase in pupil dilation as a function of cue condition (hand-cued, finger-cued, neither-cued, uncued) during the 500-2000 ms preparation interval (the interval that showed a differentiation between cue conditions), followed by Tukey LSD post-hoc tests, confirmed this picture by showing robust differences between all three cued conditions, $\mathrm{F}(3,51)=6.98$, $\eta 2=.291, \mathrm{p}<.001$. This ordering of pupil dilation as a function of cue difficulty conforms to the predictions of the Grouping Model. Moreover, as can be seen in Figure 2.2, overall, the uncued condition tended to produce the smallest increase in pupil size during the $500-2000 \mathrm{~ms}$ preparation interval, but its difference with the hand-cued condition was not statistically reliable $(p>$ $.5)$.

Finally, it is interesting to note that, after the preparation interval, the pupil continued to dilate strongly until some time after response execution (with a 
similar ordering, but less divergence, of cue conditions). This phenomenon has been observed before in a simple (1-choice) RT paradigm (Richer, Silverman, \& Beatty, 1983), and suggests that motor processes concerned with response execution and response monitoring carry a high cognitive load (Jennings \& Van der Molen, 2005).

\section{DISCUSSION}

The main outcome of this study was that difficulty of response preparation was reflected by pupil dilation. Difficulty of response preparation was manipulated by cuing two fingers on one hand (hand-cued), two homologous fingers on two hands (finger-cued), and two non-homologous fingers on two hands (neither-cued). Previous research has shown that these cuing conditions differ in cuing efficiency, with hand-cues being most efficient and neither-cues being least efficient. According to the Grouping Model (Adam, Hommel, \& Umiltà, 2003b, 2005), this pattern of differential cuing benefits is due to differences in subgroup strength, with strong or natural subgroups requiring less cognitive effort to establish and prepare than weak or ambiguous subgroups. The present finding that larger pupillary dilations were observed for the selection and preparation of more difficult response sets provides converging evidence for the Grouping Model. Moreover, this finding is consistent with the results of a recent fMRI study, which showed greater levels of brain activity for the more difficult response cues in the finger-cuing paradigm, particularly in the parietal cortex (Adam et al., 2003a).

An additional finding was that during the preparation interval, pupil dilation in the hand-cued condition was not significantly different from that in the uncued condition, even though RTs in the hand-cued condition were $58 \mathrm{~ms}$ shorter than those in the uncued condition. This outcome suggests that the preparatory process involved in hand-cues may indeed have occurred in a bottom-up, rather effortless way, which is distinct from the more effortful, top-down preparation of finger-and neither-cues. This outcome, as well as the finding that the RTs of the three cued conditions were similar but their pupil responses were not, indicates dissociation between behavioral and psychophysiological measures of response preparation, as has been observed before (Jennings \& Van der Molen, 2005; Miller, Coles, \& Chakraborty, 1996). Furthermore, it advocates caution in assuming that task processing load can be estimated directly from RT measures. As Jennings and Van der Molen (2005) have emphasized, different indices of preparation correlate poorly and 
typically do not correlate highly with performance measures, suggesting that preparation is not a single process but rather a set of processes.

Another finding was that RT in the finger-cued condition was slightly (i.e., 15 ms) shorter than RT in the hand-cued condition. A similar finding has been reported before (albeit with a longer preparation interval of 3 s; Adam et al., 2003b, Exp.1) and can be attributed to the so-called Kornblum-effect, which is the phenomenon that standard 2-choice RTs are typically shorter with a between-hands response repertoire than with a within-hands repertoire, possibly because of less response competition in a between-hands set than in a within-hands set (Kornblum, 1965). This finding does not contradict the Grouping Model, because this model explicitly acknowledges the importance of the process of within-subgroup discrimination (which follows the process of subgroup making and selective preparation) as a determinant of RTs. When, according to the Grouping Model, long-duration cues have established "cleanly" defined two-choice response sets, there should be no advantage for hand-cues but rather an advantage for finger-cues (Adam et al., 2003b). How long the duration of the cues should exactly be to produce a finger-advantage depends on procedural factors (e.g., number and duration of the preparation intervals) and the spatial layout of the stimulus and response displays.

Differentiation of pupil dilation as a function of cue difficulty did not start until about 600-700 ms after onset of the cue. This relative late differentiation of the pupil response might be related to the fact that RT tasks require timefocused preparation (Jennings and Van der Molen, 2005). Participants do not want to start preparing too soon, because maintenance of preparation over longer time periods is difficult to sustain and experienced as aversive and effortful (e.g., Niemi \& Näätänen, 1981). Hence, it is not surprising that participants show delayed pupil responses with long (e.g., $3 \mathrm{~s}$ ) as compared to short (e.g., 1 s) preparation intervals (Richer et al., 1983). In this view, the present preparation interval of $2 \mathrm{~s}$ might be considered as relatively long. Indeed, previous research with the finger-cuing task has shown that optimal preparation benefits may be achieved within $1 \mathrm{~s}$ of preparation time (Adam et al., 1998).

We now consider several alternative explanations of the preparation advantage induced by left-right or hand-cues. A first alternative account of the hand advantage is the "spatial proximity" hypothesis. According to this hypothesis, preparation for two stimulus positions is more efficient the closer 
together they are, possibly because of an advantage in sharing attention across nearby positions (Miller, 1982). This hypothesis, however, can be rejected because the observed effects do not support the explanation. That is, the spatial proximity hypothesis would predict shorter RTs when the two index fingers are cued than when the two middle fingers are cued, simply because the cue locations are in closer proximity in the former situation than in the latter. The results, however, do not support this prediction as RTs for preparing two middle or two index fingers were not significantly different $(p>$ .4). Similar findings have been reported before (e.g., Adam, 1992; Miller, 1982).

A second alternative explanation is the "dark region" hypothesis. According to this account, the visual spatial cues differ in terms of the extend and dispersion of the local dark regions they occupy on the screen, with handcues having a big dark mass on the left or right side of the display and the other cues having dark spots on both sides of the center. This account, however, does not fit with the observation that pupil dilation in the handcued and uncued conditions was similar during the preparation interval, even though they differed in terms of physical appearance and darkness (illuminance).

The third alternative account focuses on the left-right distinction in the stimulus-response displays as being critical, with the hand-distinction being of no importance (e.g., Reeve \& Proctor, 1984, 1990). From this perspective, the "hand"-advantage is due to the saliency of left-right spatial locations in the stimulus and response displays, with the specific effectors (fingers) assigned to these locations being of no or minor relevance. Using an overlapped placement of hands procedure (i.e., fingers of both hands alternating on response keys in the order: right index, left middle, right middle, left index), Reeve and Proctor (1984) reported that the usual advantage for the hand-cued condition (two fingers on one hand) turned into an advantage for the neithercued condition (two fingers on different hands). On the basis of this finding, Reeve and Proctor (1984) argued that hand-cued advantage really is an advantage for the two leftmost and two rightmost stimulus-response locations, not for the left or right hand per se. Adam et al. (2003b), however, noticed that Reeve and Proctor's result with the hand-placement manipulation might be restricted to, and thus an artifact of, two procedural factors: The task instructions provided to participants regarding the possibilities of preparation and the presentation mode of the preparation intervals. In particular, Adam et al. (2003b) observed that Reeve and Proctor (1984) did not explicitly instruct their participants to prepare all possible 
finger pairings, nor did they group the different preparation intervals together in separate blocks of trials. According to Adam et al. (2003b), this procedure might have favored the more natural left-right cues. Moreover, when Adam et al. (2003b, Experiment 5) investigated the hand-placement manipulation with more "optimal" preparation procedures (i.e., explicitly telling the participants to prepare all possible finger pairings, and grouping the different preparation intervals together in separate blocks of trials), they found that the advantage of the left-right cues disappeared. Thus, when the perceptually salient leftright cues are not combined with the anatomically based hand distinction, Reeve and Proctor's "left-right" advantage disappears. Therefore, it appears that the anatomically based hand distinction is a prerequisite for the left-right advantage to materialize and, hence, in effect can be considered a true handadvantage.

The Grouping Model's bottom-up/top-down and automatic/effortful processing distinction is consistent with several modern dual-route conceptions of response selection (Kornblum, Hasbroucq, \& Osman, 1990). According to these views, response selection can occur via (a) a slow, indirect, translation route that applies a translation rule and draws upon central resources, and/or (b) a fast, direct, automatic response activation route that exploits natural and coherent stimulus-response links. Also, the Grouping Model is consistent with a recent, thorough review of the response preparation literature by Jennings and Van der Molen (2005), who identified several key features of response preparation, including its effortful and cognitive nature and its dependence on the complexity of the motor task. The present findings reinforce this framework and indicate that physiological changes during response preparation as indexed by changes in pupil size can be used to test a theory of response preparation.

Finally, it is relevant to note that the present study is not the first to examine pupillary responses during preparation for speeded action. However, whereas previous studies typically used a simple RT time paradigm (one target stimulus, one pre-specified response) and focused on the effects of stimulus uncertainty (e.g., Bradshaw, 1968; Richer et al., 1983; Jennings et al., 1998; Bitsios et al., 2004), the present study used a four-choice RT task and focused on the effects of response uncertainty. The consistent finding across all these studies is that greater uncertainty is associated with a reduced pupillary response, and that anticipation of the stimulus event and advance selection of the appropriate response both lead to an increased pupillary response. Hence, our study extends previous work that used the pupillary response as 
an index of processing load by showing that it is also sensitive to manipulations of task difficulty in the domain of response preparation.

\section{REFERENCES}

Adam, J. J. (1992). The spatial proximity hypothesis of the hand advantage in spatial precuing tasks. Human Movement Science, 11, 641-652.

Adam, J. J., Backes, W., Rijcken, J., Hofman, P., Kuipers, H., \& Jolles, J. (2003a). Rapid visuomotor preparation in the human brain: a functional MRI study. Cognitive Brain Research, 16(1), 1-10.

Adam, J. J., Hommel, B., \& Umiltà, C. (2003b). Preparing for perception and action (I): the role of grouping in the response-cuing paradigm. Cognitive Psychology, 46(3), 302-358.

Adam, J. J., Hommel, B., \& Umiltà, C. (2005). Preparing for perception and action (II) Automatic and effortfull Processes in Response cuing. Visual Cognition, 12(8), 1444-1473.

Adam, J. J., Paas, F. G., Teeken, J. C., van Loon, E. M., van Boxtel, M. P., Houx, P. J., et al. (1998). Effects of age on performance in a finger-precuing task. Journal of Experimental Psychology Human Perception and Performance, 24(3), 870-883.

Adam, J. J., Paas, F. G. W. C., Teeken, J. C., van Loon, E. M., van Boxtel, M. P., Houx, P. J., et al. (1998). Effects of age on performance in a finger-precuing task. Journal of Experimental Psychology Human Perception and Performance, 24(3), 870-883.

Allen, P. A., Groth, K. E., Weber, T. A., \& Madden, D. J. (1993). Influence of response selection and noise similarity on age differences in the redundancy gain. Journal of Gerontology, 48(4), P189198.

Andreassi, J. L. (2000). Pupillary response and behaviour. In N. L. E. A. Mahwah (Ed.), Psychophysiology. Human behaviour and Physiological response (4th ed., Vol. 10, pp. 218-233). New York: Oxford University Press.

Beatty, J., \& Lucero-Wagoner, B. (2000). The Pupillary system. In J. T. Cacioppo, Tassinary, L.G. \& Berntson, GB (Ed.), Handbook of Psychophysiology (2nd ed., pp. 142-162). Cambridge: Cambridge University Press.

Bitsios, P., Szabadi, E., \& Bradshaw, C. M. (2004). The fear-inhibited light reflex: importance of the anticipation of an aversive event. International Journal of Psychophysiology, 52(1), 87-95.

Bradshaw, J. L. (1968). Pupillary changes and reaction time with varied stimulus uncertainty. Psychonomic Science, 13(2), 69-70.

Brainard, D. H. (1997). The Psychophysics Toolbox. Spatial Vision, 10(4), 433-436.

Cornelissen, F. W., Peters, E. M., \& Palmer, J. (2002). The Eyelink Toolbox: eye tracking with MATLAB and the Psychophysics Toolbox. Behavior Research Methods, Instruments \& Computers, 34(4), 613-617.

Hasher, L., \& Zacks, R. T. (1979). Automatic and effortful processes in memory. Journal of Experimental Psychology, 108, 356-388.

Hellige, J. B. (1993). Hemispheric Asymetry: What's Right and What's Left. Cambridge, Massachusetts. London, England.: Harvard University Press. .

Hick, W. E. (1952). On the rate of gain of information. Quarterly Journal of Experimental Psychology, 4, 11-26.

Hyman, R. (1953). Stimulus information as a determinant of reaction time. Journal of Experimental Psychology, 45(3), 188-196.

Jennings, J. R., \& Van der Molen, M. W. (2005). Preparation for speeded action as a psychophysiological concept. Psychological Bulletin, 131(3), 434-459.

Jennings, J. R., van der Molen, M. W., \& Steinhauer, S. R. (1998). Preparing the heart, eye, and brain: foreperiod length effects in a nonaging paradigm. Psychophysiology, 35(1), 90-98. 
Jin, P. (1992). Toward a reconceptualization of the law of initial value. Psychological Bulletin, 111(1), 176-184.

Kornblum, S. (1965). Response competition and/or inhibition in two-choice reaction time. Psychonomic Science, 255-56.

Kornblum, S., Hasbroucq, T., \& Osman, A. (1990). Dimensional overlap: cognitive basis for stimulusresponse compatibility--a model and taxonomy. Psychoogiall Review, 97(2), 253-270.

Mapp, A. P., \& Ono, H. (1999). Wondering about the wandering cyclopean eye. Vision Research, $39(14), 2381-2386$.

Miller, J. (1982). Discrete versus continuous stage models of human information processing: in search of partial output. Journal of Experimental Psychology Human Perception and Performance, 8(2), 273-296.

Miller, J., Coles, M. G., \& Chakraborty, S. (1996). Dissociation between behavioral and psychophysiological measures of response preparation. Acta Psychologica (Amst), 94(2), 189208.

Nicoletti, R., \& Umilta, C. (1989). Splitting visual space with attention. Journal of Experimental Psychology Human Perception and Performance, 15(1), 164-169.

Niemi, P., \& Näätänen, R. (1981). Foreperiod and simple reaction time. Psychological Bulletin, 89, 133-162.

Proctor, R. W., \& Reeve, T. G. (1986). A caution regarding use of the hint procedure to determine whether partial stimulus information activates responses. Perception and Psychophysics, 40(2), $110-118$.

Reeve, T. G., \& Proctor, R. W. (1984). On the advance preparation of discrete finger responses. Journal of Experimental Psychology Human Perception and Performance, 10(4), 541-553.

Reeve, T. G., \& Proctor, R. W. (1990). The salient-features coding principle for spatial-and symboliccompatibility effects. In R. W. Proctor \& T. G. Reeve (Eds.), Stimulus-response compatibility (pp. 163-180). Amsterdam: North-Holland.

Reeve, T. G., Proctor, R. W., Weeks, D. J., \& Dornier, L. (1992). Salience of stimulus and response features in choice-reaction tasks. Perception and Psychophysics, 52(4), 453-460.

Richer, F., Silverman, C., \& Beatty, J. (1983). Response selection and initiation in speeded reactions: a pupillometric analysis. Journal of Experimental Psychology Human Perception and Performance, 9(3), 360-370.

Rosenbaum, D. A. (1991). Human Motor Control. New York: Academic Press.

Van Gerven, P. W. M., Paas, F., Van Merriënboer, J. J. G., \& Schmidt, H. G. (2004). Memory load and the cognitive pupillary response in aging. Psychophysiology, 41, 167-174.

\section{Acknowledgements}

We would like to acknowledge Paul Willems, Anne Roefs, Huub Hamers, Jacco Ronner, Johan Gielissen, Ron Hellebrand, Michiel Vestjens, Marianne Severens and Bart Ament for providing their support to this study. 


\section{RESPONSE PREPARATION WITH ADJACENT VERSUS OVERLAPPED HANDS CHAPTER 3}

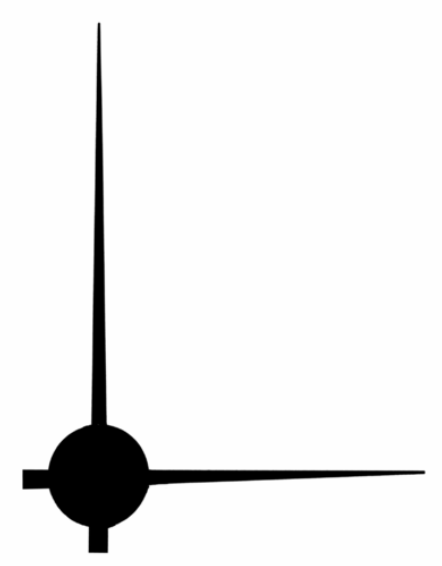

Sofie Moresi, Jos J. Adam, Jons Rijcken, Harm Kuipers \& Pascal W.M. Van Gerven.

Response Preparation with Adjacent versus Overlapped hands:

a pupillometric study.

(Submitted) 


\section{ABSTRACT}

This study investigated the effect of overlapping the hands on response preparation in a group of forty participants who performed the finger-cuing task with the hands adjacent and overlapped. We measured the pupillary response to assess the cognitive processing load associated with different types of response preparation. Results showed stronger pupil dilations (and also longer reaction times and more errors) for the overlapped than for the adjacent hand placement condition, reflecting an overall increase in cognitive processing load. Importantly, the negative impact of overlapping the hands on pupil dilation significantly interacted with cue type, with left-right cues showing the strongest effects. This interaction effect demonstrates the importance of motoric factors in response preparation, which confirms the prediction of the Grouping Model.

\section{INTRODUCTION}

About 25 years ago, Miller (1982) reported that preparing two fingers on one hand is more effective than preparing two fingers on different hands. Interpretation of this (same) hand-advantage phenomenon has sparked much debate. Miller (1982) attributed the hand-advantage to characteristics of the motor system, arguing that it is consistent with the lateralized control of hand and finger movements in the cerebral cortex. Reeve and Proctor (1984, 1985), however, questioned Miller's motoric interpretation, contending that the hand-advantage is an artifact of nonmotoric decision processes involved in translating between stimuli and responses. More recently, Adam, Hommel and Umiltà $(2003,2005)$ asserted that the hand-advantage is due to a combination of factors, attentional, perceptual, and motoric.

In this study, we attempted to shed new light on this debate by examining the pupillary response as an index of cognitive processing load in response preparation. Our key manipulation was that of hand placement (adjacent vs. overlapped), which dissociates hand and spatial position factors that are confounded with the hands adjacent (Reeve \& Proctor, 1984). The pupillary response supplements the traditional performance measures of reaction time (RT) and response accuracy (error rate) by providing a reliable, on-line psychophysiological measure of processing load during many cognitive tasks (for a review, see Beatty \& Lucero-Wagoner, 2000), including response 
preparation (Moresi et al., 2008). Before explicating the main theoretical frameworks, we briefly introduce the methodology of the finger-cuing paradigm and its key findings.

\section{The Finger-Cuing Paradigm}

In the finger-cuing task developed by Miller (1982), a visual cue appears before the onset of the target stimulus, allowing the advance preparation of a subset of two out of four possible finger (key press) responses. Typically, the hands are placed adjacently, with the index and middle fingers of both hands resting on a linear array of four keys. In the present study, the visual display consisted of a horizontal row of four white boxes centered on a computer monitor. A trial started with the presentation of these four white boxes. After $1 \mathrm{~s}$, the cue signal was presented by coloring two boxes grey, which allowed the selective preparation of two finger responses indicated by the cue. Then, after a delay of $2 \mathrm{~s}$, the target stimulus was presented by coloring one of the two grey boxes black, which signaled the required finger response. The $2 \mathrm{~s}$ interval between onset of the cue signal and onset of the target signal is called the preparation interval, as it reflects the available amount of time to prepare the two finger responses indicated by the cue before the target appears. Note that whereas the present study used only one preparation interval of $2 \mathrm{~s}$ (to allow sufficient time for the pupil to dilate), many previous studies used a range of preparation intervals (e.g., 0, 375, 750, 1500, and 3000 $\mathrm{ms}$; Reeve \& Proctor, 1984) to study the time course of response preparation. Four cue or preparation conditions are distinguished (see Figure 2.1). In the left-right cue condition (hand-cued), the cue indicates the two locations assigned to either the left hand (the two leftmost locations) or right hand (the two rightmost locations). In the inner-outer cue condition (finger-cued), the cue specifies the two locations assigned to either the index fingers (the two inner locations) or middle fingers (the two outer locations). In the alternate cue condition (neither-cued), the cue indicates locations assigned to the index finger of one hand and the middle finger of the other hand (the two pairs of alternate locations). These three preparation conditions are called the "cued" conditions. Also, an "uncued" control condition is included, which does not provide advance information about the upcoming response (all four boxes turn grey). In other words, the uncued condition leaves the basic fourchoice task unaltered, and, thus, is a control or baseline condition against which the effects of the "cued" conditions can be evaluated. Since RTs in twochoice tasks are substantially shorter than RTs in four-choice tasks (Hick, 1952; Hyman, 1953), cue effectiveness is inferred from a significant RT advantage or 
benefit for the two-choice "cued" conditions (i.e., left-right, inner-outer, and alternate) over the four-choice "uncued" condition.

The key, and often replicated, finding from the finger-cuing paradigm is a pattern of differential cuing benefits: RTs are shortest for the left-right cue, longest for the alternate cue, and intermediate for the inner-outer cue, reflecting an ordering in terms of preparation difficulty (Proctor \& Reeve, 1986, 1988; Reeve \& Proctor, 1984, 1990). This pattern of differential cuing benefits is primarily present with short preparation intervals (i.e., intervals of less than about $3 \mathrm{~s}$ ). When the preparation interval is $3 \mathrm{~s}$ or more, the three cued conditions often show comparable RTs and thus similar RT benefits compared to the uncued condition. This finding indicates that response preparation is a gradual process that develops over time, with left-right cues prompting a more efficient and faster response preparation process than inner-outer and alternate cues.

\section{Salient-Features Coding Principle}

Proctor and Reeve (1986, 1988; Reeve et al., 1992) interpreted the pattern of differential cuing benefits in terms of the salient-features coding principle. This principle assumes that stimulus and response sets are coded in terms of the salient features of each, with response selection occurring most rapidly when the salient features of the respective sets correspond. With spatial location stimuli, spatial coding predominates, with the left-right spatial distinction being the most salient feature. Hence, there is an advantage for left-right cues over inner-outer and alternate cues.

Reeve and Proctor (1984) obtained critical evidence for their spatial coding account by investigating the fate of the left-right advantage with an overlapped placement of hands (i.e., the index and middle fingers of both hands alternate on the response keys in the order right index, left middle, right middle, left index). This overlapped hand placement (see Figure 3.1) dissociates hand and spatial position factors that are confounded with the usual adjacent hand placement. 


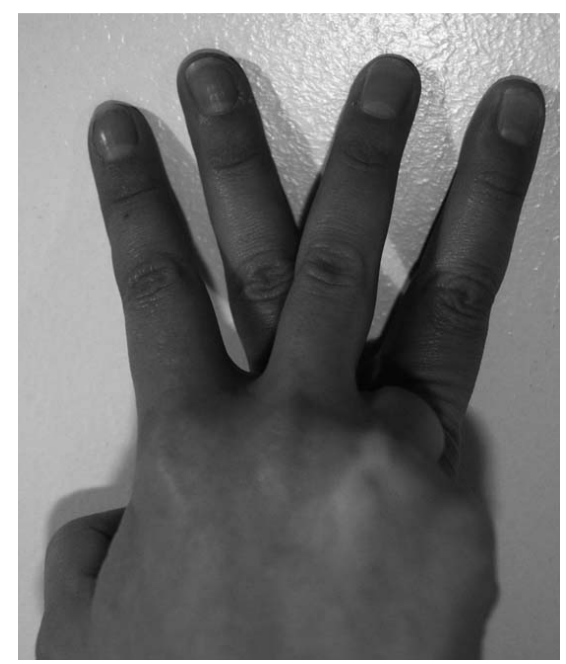

Figure 3.1. Illustration of the overlapped hand placement. Fingers of different hands are alternated (right index finger, left middle finger, right middle finger and left index finger).

Comparing finger-cuing effects with the adjacent and overlapped hand placements, Reeve and Proctor (1984, Experiment 3) found that the advantage for left-right cues is preserved with the hands overlapped, suggesting that the advantage reported by Miller (1982) really is an advantage for the two leftmost and two right-most stimulus-response locations, not for fingers on left or right hands. According to Reeve and Proctor (1984, 1985, 1990) this finding implicates the stimulus-response translation stage as the locus of Miller's "hand-advantage", which in Reeve and Proctor's view actually is a "left-right" advantage.

\section{Grouping Model}

Adam and co-workers (Adam et al., 2003, 2005; Adam \& Van Veggel, 1992) observed that Reeve and Proctor's result with the hand-placement manipulation might be restricted to, and thus an artifact of, two procedural factors: The task instructions for participants regarding the possibilities for and benefits of preparation, and the presentation mode of the preparation intervals. In particular, Reeve and Proctor (1984, Exp.1, Exp.3) did not explicitly instruct their participants to prepare all possible finger pairings, nor did they group the different preparation intervals together in separate blocks of trials. According to Adam and Van Veggel (1992), this procedure might have favored the more natural, easy to prepare left-right cues, especially at short preparation intervals. When Adam and Van Veggel (1992) manipulated the above two procedural factors, they found that when participants are explicitly instructed to prepare all possible finger pairings, and when they know in 
advance how long the preparation interval is (because of a blocked presentation mode), the relative advantage of the left-right cues over the inner-outer cues was greatly reduced (by about $60 \%$ ). This is the case because under these task constraints the inner-outer cues produced a significant RT benefit also with the shorter preparation intervals. Hence, Adam and Van Veggel (1992) concluded that response preparation has an important strategic component: Participants preferably engage in preparation activities when procedural constraints make it apparent, convenient and/or important to do so (see also, Requin, 1980).

Importantly, when Adam et al. (2003, Experiment 5) investigated the handplacement manipulation (adjacent vs. overlapped) with the more explicit preparation procedures (i.e., explicitly telling the participants to prepare all possible finger pairings, and grouping the different preparation intervals together in separate blocks of trials), the advantage of the left-right cues present with the adjacent hand placement disappeared with the overlapped hand placement. This outcome is not in accordance with results of the Reeve and Proctor studies (1984; Proctor \& Reeve, 1988) and inconsistent with a pure spatial coding account of finger-cuing effects. Instead, it supports the idea that motoric factors play a major role in finger-cuing effects, a notion endorsed by the Grouping Model (Adam et al., 2003, 2005).

The Grouping Model is an extension of the salient-features coding principle. It recognizes the importance of organizational correspondence between stimulus and response features, but in addition emphasizes the critical role of motoric (i.e., anatomical) and attentional factors in response preparation. Thus, the Grouping Model assumes that the individual elements of multielement visual displays and multi-element response arrays are not processed independently but are preattentively organized or "grouped" according to low-level grouping factors (e.g., Gestalt principles) and on response-related factors (e.g., inter-response dependencies). According to the Grouping Model, the processing advantage of the left-right cue simply reflects the natural and stronger grouping of the two leftmost and two rightmost stimulus and response elements. Each stimulus set and each response set has a default organization set up preattentively by the bottom-up computation of perceptual and motor units or subgroups. This process is fast and automatic. With additional, top-down processing, however, alternative organizations can be accomplished, which is a slow and effortful process. Thus, the pattern of cuing effects that emerges in the finger-cuing task critically relies on the nature of these default groupings and on the time available to reorganize 
them, when required. Thus the Grouping Model maintains that inner-outer cues and alternate cues are the more difficult cues because they require slow, effortful, top-down processing to break up the default, left-right spatial organization of the perceptual-motor workspace in order to create a new (perceptual and motor) organization based on the characteristics of the cue.

According to the Grouping Model, the alternate cue is more difficult than the inner-outer cue, because the former represents a perceptually asymmetrical subgroup that indicates the selection and preparation of two different, nonhomologous fingers on two hands, whereas the latter represents a perceptually symmetrical subgroup that specifies the same or homologous fingers on two hands. Because homologous fingers are neurally and functionally linked (Rosenbaum, 1991), they are easier to group than nonhomologous fingers.

\section{Aim of Study and Predictions}

The primary aim of this study was to provide a further test of the Grouping Model by investigating the effect of overlapping the hands on pupil dilation in the finger-cuing task. In previous work, we have shown that the increase in pupil size during response preparation with adjacent hands is systematically related to cue difficulty, with the left-right cues showing the smallest increase and the alternate cues showing the greatest increase (Moresi et al., 2007). With the hands overlapped, the Grouping Model, with its emphasis on anatomical coding, would predict a strong negative influence of overlapping the hands on cuing efficiency, especially for left-right cues. This is because overlapping the hands greatly reduces the good grouping of the two leftmost and two rightmost response elements in the response set (they lose the hand distinction), while leaving the inner-outer and alternate groupings relatively intact. Consequently, according to the Grouping Model, overlapping the hands should disproportionally increase the processing load of left-right cues, thereby reducing or eliminating the pattern of differential pupil dilations.

\section{METHOD}

\section{Participants}

Participants were 40 right-handed students at Maastricht University (22 women, 18 men; mean age $=21.5$ years, $\mathrm{SD}=2.3$ ). All had normal or correctedto-normal vision. None of them took eye medication that could influence the 
pupillary response. Informed consent was obtained and they received $€ 7.50$ for their participation. One female participant was excluded from the sample due to $60 \%$ pupil artifacts.

\section{Stimuli and apparatus}

The visual display consisted of a row of four white boxes $(0.5 \times 0.5 \mathrm{~cm})$ in black outline, presented on a standard computer screen with a grey background, which corresponded to the calibration background. The two centre boxes were separated by $1.0 \mathrm{~cm}$, and the two leftmost and two rightmost boxes were separated by $0.5 \mathrm{~cm}$ (side-to-side). Participants were seated in a heightadjustable chair with their heads stabilized in a chinrest placed at a distance of $57 \mathrm{~cm}$ from the monitor. Participants placed the index and middle fingers of both hands on four linearly arrayed push buttons mounted on a response box, placed in front of the subjects on a table, and aligned with the centre of the stimulus set. The illumination in the room was constant (about $600 \mathrm{~lx}$ ). RTs were measured in milliseconds. The software controlling the RT measurements and the stimulus presentation was programmed in Matlab 7 and supported by the Eyelink Toolbox (Brainard, 1997; Cornelissen et al., 2002). The pupil area was measured in pixels by the Eyelink I Gazetracker (SR Research Ltd., Canada), a head-mounted infrared video-based tracking system with a temporal resolution of $250 \mathrm{~Hz}$ and a spatial resolution of at least $0.01^{\circ}$.

\section{Task}

A trial started with the presentation of the four white boxes (warning signal). After a delay of $1 \mathrm{~s}$, the cue was presented for $2 \mathrm{~s}$ by coloring two of the four boxes grey. Then, the target stimulus was presented by coloring one of the two grey boxes black, signaling the required finger response. After the finger response, there was an inter-trial interval of $3 \mathrm{~s}$ before the warning signal of the next trial appeared.

\section{Design and Procedure}

All participants performed the finger cuing task in two hand placement conditions. In the adjacent hand placement condition, the hands were placed adjacently to each other. In the overlapped hand placement condition, the fingers of different hands alternated, so that the placement of fingers from left to right was as follows: right index finger, left middle finger, right middle finger, and left index finger. This arrangement was identical to the overlapped hand placement condition used by Adam et al. (2003, Experiment 5) and Reeve and Proctor (1984, Experiment 3). Half of the participants $(n=20)$ performed with their right hand on top of the left hand, whereas the other half 
performed with their left hand on top of the right hand. Furthermore, half of the participants started with the adjacent condition followed by the overlapped condition (adjacent first), while the other half began with the overlapped condition (overlapped first).

Each participant received one block of 80 test trials for each hand placement condition (each preceded by 16 practice trials), separated by a short break of 1 min, making a total of 160 test trials. Within a series of 80 trials, there were 20 trials for each cue condition (left-right, inner-outer, alternate, and uncued) presented in random order. Participants were informed about the nature of the task and were explicitly instructed to take advantage of the informative cues by preparing the cued responses. They were instructed to respond as quickly and accurately as possible to the target stimulus by pressing the correct response key. No feedback was provided. At the start of each individual test session, a nine-point calibration and validation procedure of the Eyelink I Gazetracker was performed.

\section{Analyses}

Trials with RTs shorter than $125 \mathrm{~ms}$ or longer than 1,250 ms (2.3\%) were considered outliers and therefore excluded from all data analyses. Mean correct RTs and proportions of errors were calculated for each participant as a function of hand placement (adjacent, overlapped), cue condition (uncued, left-right, inner-outer, and alternate) and hand placement order (starting with adjacent or overlapped hand placement). Pupil data from the left eye were analyzed and only for correct trials (i.e., trials without response errors and RT outliers). Eye blinks were filtered out by a computer algorithm and missing pupil data were replaced by cubic spline interpolation, starting 17 samples (68 $\mathrm{ms}$ ) before and ending 25 samples (100 ms) after the blink ( $9 \%$ dropout). For each trial, a baseline pupil area was determined by calculating the average pupil size during a period of $500 \mathrm{~ms}$ preceding the onset of the cue signal. Cue-locked pupil dilation was calculated by subtracting this baseline area from the pupil area for each data point during a period of $5 \mathrm{~s}$ following cue onset. This difference score was converted to a percentage of the corresponding baseline value. This conversion is warranted by Jin's (1992) revised version of the so-called "Law of Initial Value" (LIV), which states that within the middle range of baseline values, the physiological response increases as a function of baseline. This version of the LIV was applicable to our data, because there was a positive overall correlation between baseline pupil size and peak dilation $(r=.70)$. The LIV introduces a physiological artifact, however, because it influences pupil dilation and thus may obscure 
the effect of cue condition. Expressing dilation as a percentage of the baseline corrects for this artifact, because pupillary responses following a relatively high baseline value are attenuated. This procedure was earlier applied by, for example, Van Gerven et al. (2004). An alpha level of .05 was used to determine statistical significance.

\section{RESULTS}

\section{Reaction Times}

A mixed analysis of variance (ANOVA) was performed on mean RT with hand placement (adjacent, overlapped) and cue condition (uncued, left-right, inner-outer, alternate) as within-subjects variables, and hand placement order (adjacent first, overlapped first) as the between-subjects variable. The significant main effect of hand placement, $F(1,37)=102.36, p<.001$, indicated much longer RTs with the hands overlapped than with the hands adjacent (523 ms vs. $413 \mathrm{~ms}$, respectively).The significant main effect of cue condition, $\mathrm{F}(3,111)=120.15, \mathrm{p}<.001$, reflected substantial and reliable preparation benefits for all three cued conditions compared to the uncued condition (452 $\mathrm{ms}, 442 \mathrm{~ms}, 430 \mathrm{~ms}$, and $550 \mathrm{~ms}$ for left-right, inner-outer, alternate, and uncued, respectively). Performing the adjacent hand placement condition first produced shorter RTs than performing the overlapped hand placement first (454 ms vs. $483 \mathrm{~ms}$, respectively), but this difference was statistically not reliable, $F(1,37)=1.92, p>.15$.

The significant interaction between hand placement and cue condition, $F(3$, $111)=23.14, p<.001$, reflected greater cuing benefits with the hands overlapped than with the hands adjacent ( $142 \mathrm{~ms}$ vs. $76 \mathrm{~ms}$, respectively). As can be seen in Figure 3.2, this occurred because overlapping the hands lengthened RT more in the uncued condition than in the cued conditions. Furthermore, this interaction indicated that with the hands placed adjacent the three cued conditions (left-right, inner-outer, alternate) showed similar RTs, $F(2,76)=1.97, p>.1$, whereas with the hands overlapped they showed different RTs, $F(2,76)=7.29, p<.001$, with the alternate cue condition showing significantly shorter RTs than the left-right and inner-outer cue conditions (466 ms, 504 ms, and 495 ms, respectively; Tukey's Least Significant Difference post-hoc test). Finally, the significant three-way interaction between hand placement, cue condition, and hand placement order, $F(3,111)=3.88, p<.05$, indicated that the negative effect of overlapping the hands on RTs in the 
uncued condition was greatest when performing the adjacent hand placement first.

\section{Errors}

Overall mean error rate was $3.9 \%$. Participants made more errors with the hands overlapped than with the hands adjacent ( $6.8 \%$ vs. $1.2 \%$, respectively), $F(1,37)=29.66, p<.001$. Furthermore, participants made fewer errors in the left-right cue condition than in all other conditions $(2.5 \%, 4.3 \%, 4.3 \%$, and $4.5 \%$ for left-right, inner-outer, alternate, and uncued, respectively), $F(3,111)=$ $3.6, \mathrm{p}<.05$. No significant interactions were observed ( $\mathrm{ps}>.5$ ). 

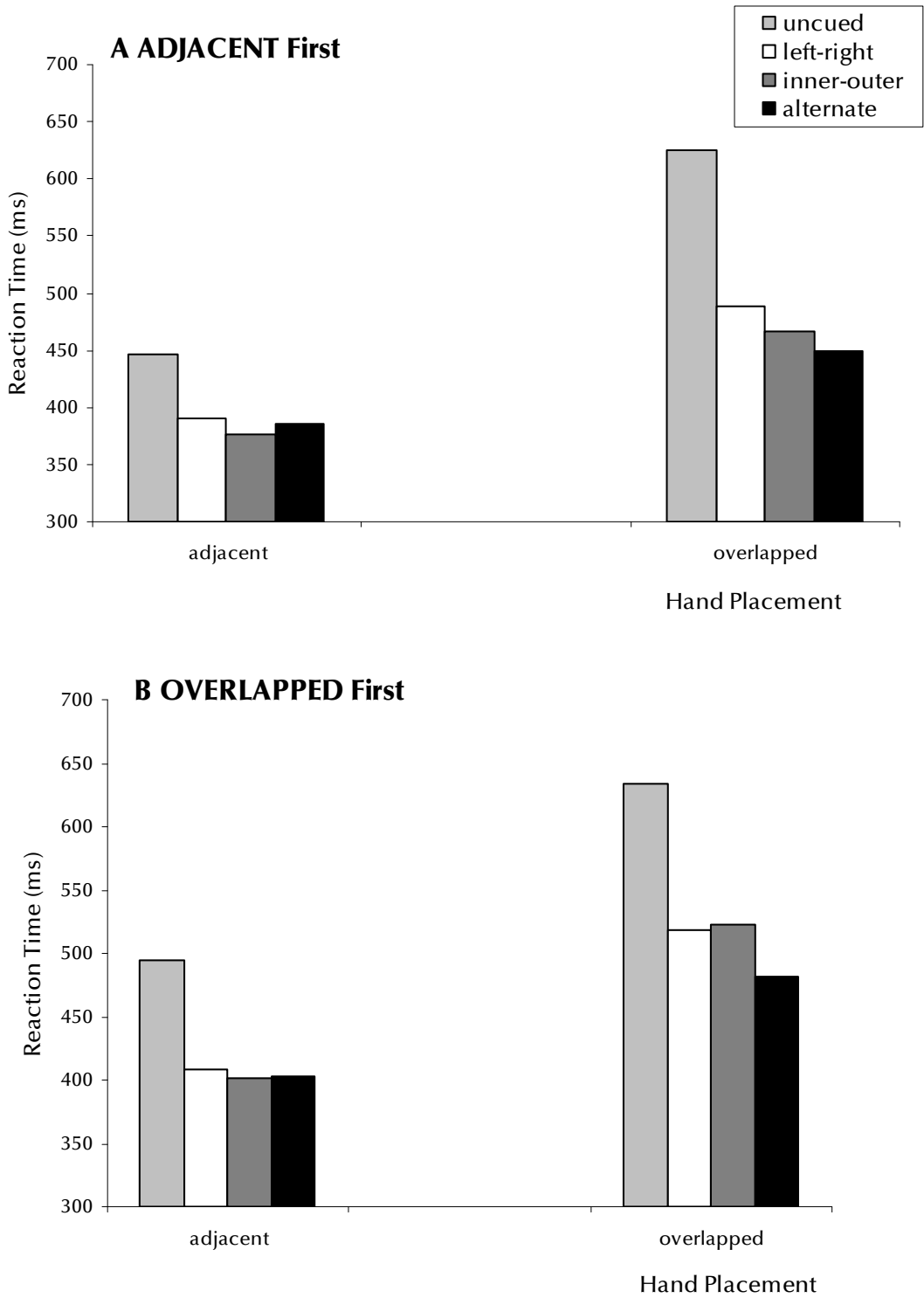

Figure 3.2. Mean reaction time ( $\mathrm{ms})$ as a function of cue condition and hand placement for the adjacent-first (panel $A$ ) and overlapped-first (panel B) hand placement orders. 


\section{Pupil dilation}

Figure 3.3 shows mean pupil dilation as a function of time and cue condition for the adjacent and overlapped hand placement conditions (for the adjacent hands first order). Figure 3.4 shows the same data for the overlapped hands first order. As can be seen in Figure 3.3, the pupil started to dilate at about 450 ms after cue onset, followed by a clear differentiation of the dilation curves for the cued conditions at about $1,000 \mathrm{~ms}$. To analyze the pupil data statistically, we calculated mean pupil dilation as a function of hand placement, cue condition, and hand placement order for the 1,000-2,000 ms interval after cue onset, and entered these in a 2 (hand placement) x 4 (cue condition) $\times 2$ (hand placement order) mixed ANOVA.

The significant main effect of hand placement, $F(1,37)=45.37, p<.001$, indicated a stronger mean pupil dilation for the overlapped hand placement than for the adjacent hand placement $(7.0 \%$ vs. $2.7 \%$, respectively). The significant main effect of cue condition, $F(3,111)=27.69, \mathrm{p}<.001$, indicated a stronger pupil dilation for the three cued conditions than for the uncued condition $(5.6 \%, 5.1 \%, 6.7 \%$, and $1.9 \%$ for left-right, inner-outer, alternate, and uncued, respectively). The significant main effect of hand placement order, $\mathrm{F}(1,37)=4.19, \mathrm{p}<.05$, indicated a stronger pupil dilation for the adjacent-first order than for the overlapped-first order $(5.8 \%$ vs. $3.8 \%$, respectively). Moreover, hand placement order interacted with hand placement, $F(1,37)=$ $4.78, p<.05$, indicating that the increase in pupil dilation due to overlapping the hands was greater for the adjacent-first order than for the overlapped-first order $(5.7 \%$ vs. $3.0 \%$, respectively). Importantly, there was a significant hand placement $x$ cue condition interaction, $F(3,111)=5.96, p<.001$, which indicated that overlapping the hands caused the greatest increase in pupil dilation for left-right cues (an increase of $6.0 \%$ ), an intermediate increase for inner-outer and alternate cues (both $4.7 \%$ ), and the smallest increase for uncued $(2.0 \%)$. Although there was no significant three-way interaction between hand placement, cue condition, and hand placement order $(F(3,111)$ $=0.83, p>.4)$, the patterns of pupil dilation as a function of hand position and cue condition appeared to differ for the respective hand placement orders, which we therefore analyzed separately. 

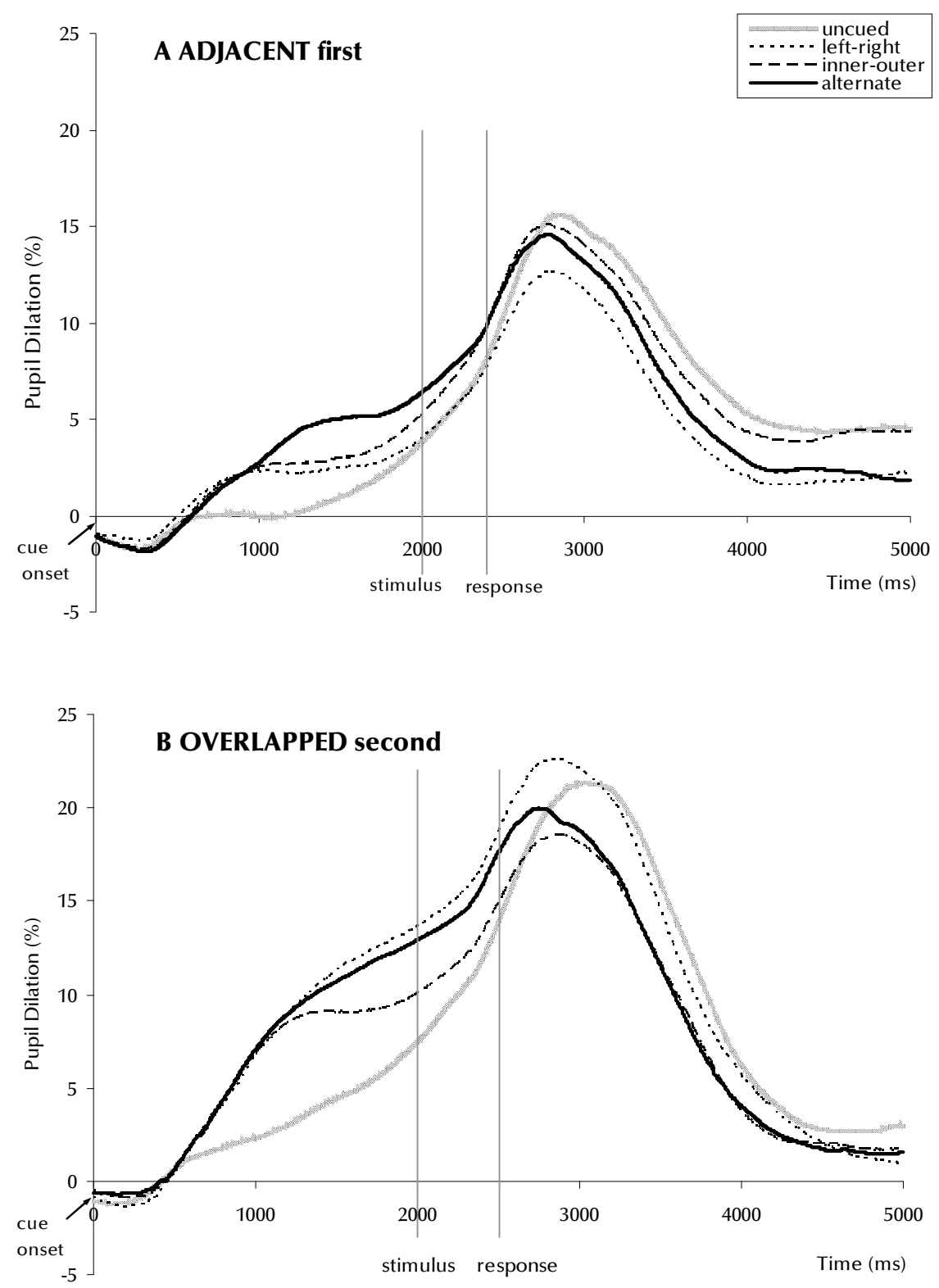

Figure 3.3. The dilation-by-time plots in the adjacent first hand placement order for the four cue conditions, starting from cue onset until $5 \mathrm{~s}$ afterwards. The stimulus is presented for 2 $\mathrm{s}$ after cue onset and the response averagely occurs at $\mathbf{4 0 0} \mathbf{~ m s}$ after stimulus onset for adjacent (panel A) hand placement and at $507 \mathrm{~ms}$ after stimulus onset for the overlapped hand placement (panel B). 


\section{Adjacent first, overlapped second}

A 2 (hand placement) $\times 4$ (cue condition) repeated-measures ANOVA yielded main effects of hand placement, $F(1,18)=30.18, p<.001$, and cue condition, $F(3,54)=12.86, p<.001$, and a significant interaction, $F(3,54)=3.31, p<.05$. This interaction indicated that the usual pattern of differential pupil dilations for the three cued conditions with the hands placed adjacent (least pupil dilation for the left-right cue, most pupil dilation for the alternate cue) turned into a different pattern with the hands overlapped, that is, smallest pupil dilation for the inner-outer cue and greatest pupil dilation for the left-right cue (see Figure 3.3). In other words, whereas the left-right cue condition showed the smallest pupil dilation with the hands adjacent $(2.6 \%)$, it showed the largest pupil dilation with the hands overlapped $(10.9 \%)$, a large, significant increase of $8.3 \%$. Inner-outer and alternate cue conditions showed smaller pupil dilation increments for overlapped compared to adjacent hand placements $(5.6 \%$ and $5.7 \%$, respectively). Thus, for the adjacentfirst/overlapped-second order, the effect of overlapping the hands on pupil dilation was strongest for the left-right cue condition.

\section{Overlapped first, adjacent second}

For this hand placement order, the repeated-measures ANOVA also revealed main effects of hand placement, $F(1,19)=14.57, p<.001$, and cue condition, $F(3,57)=15.04, p<.001$, as well as a significant interaction, $F(3,57)=3.40, p<$ .05. The interaction reflected a larger increase in pupil dilation due to overlapping the hands for the three cued conditions than for the uncued condition, but, importantly, there was no differential effect among the three cued conditions. That is, left-right, inner-outer, and alternate cue conditions showed similar increments in pupil dilation due to overlapping the hands (i.e., $3.7 \%$, 3.7\%, and 3.6\%, respectively; see Figure 3.4 ). 

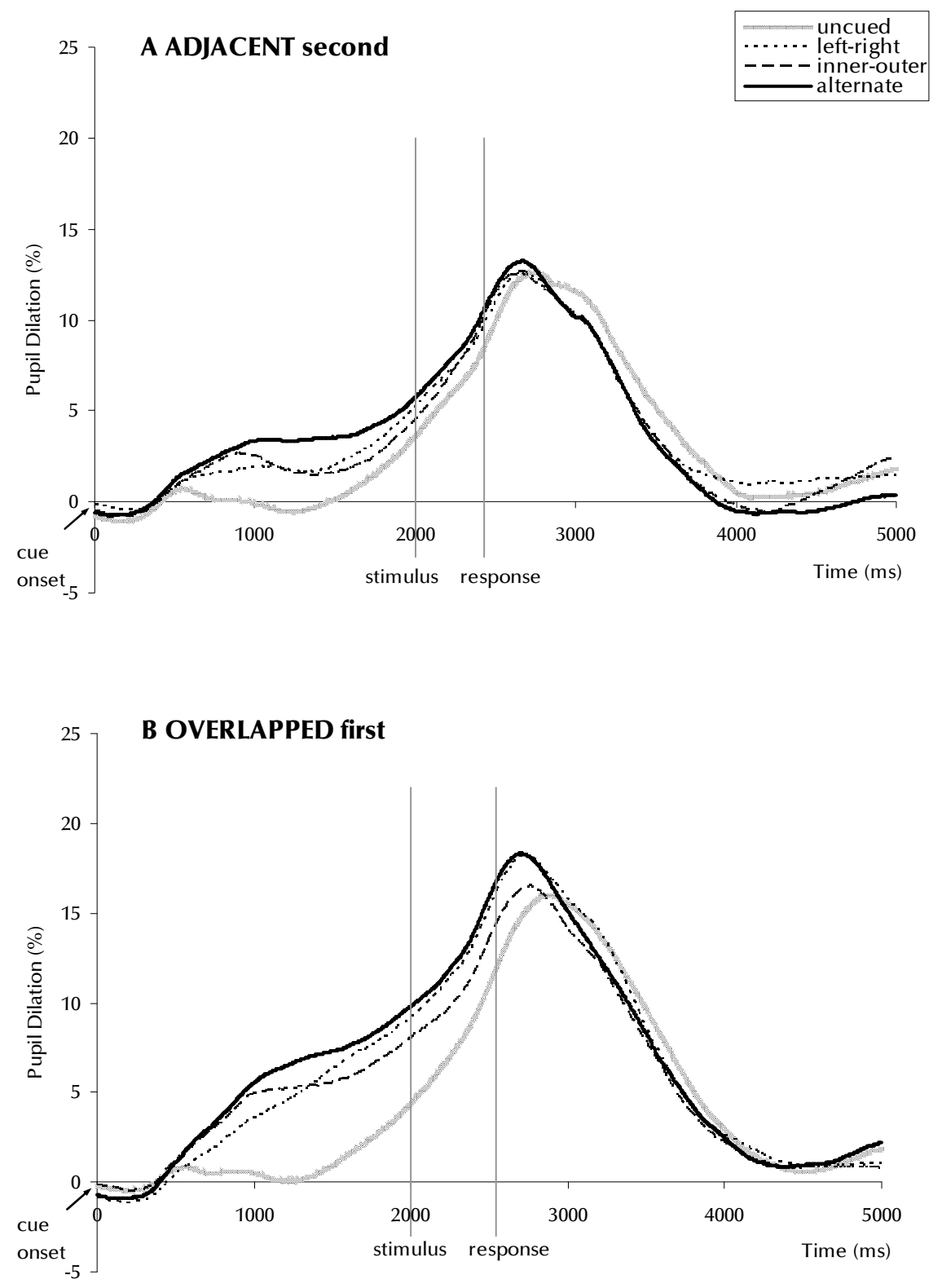

Figure 3.4. The dilation-by-time plots in the overlapped first hand placement order for the four cue conditions, starting from cue onset until $5 \mathrm{~s}$ afterwards. The stimulus is presented for 2 $\mathrm{s}$ after cue onset and the response averagely occurs at $\mathbf{4 2 7} \mathbf{~ m s}$ after stimulus onset for adjacent (panel A) hand placement and at $539 \mathrm{~ms}$ after stimulus onset for the overlapped hand placement (panel B). 


\section{DISCUSSION}

In this study we evaluated predictions derived from the Grouping Model (Adam et al., 2003, 2005) regarding the effect of overlapping the hands on response preparation. To this end we recorde the pupil response in a fingercuing task with a preparatory interval of $2 \mathrm{~s}$ and used two different hand placements (adjacent vs. overlapped). Results showed a significant mediating influence of the hand placement manipulation on both cuing efficiency (indexed by RT and error rate) and cognitive processing load (indexed by pupil dilation). In particular, RT results showed a pattern of equivalent RTs for the left-right, inner-outer, and alternate cues with the hands adjacent, but a pattern of differential RTs with the hands overlapped, with alternate cues now associated with two fingers on the same hand - producing the shortest RTs. Pupil dilation results showed that with the hands adjacent pupil dilation was smaller for left-right cues than for alternate cues, but with the hands overlapped this advantage for left-right cues disappeared. These two findings converge on the conclusion that the anatomical hand distinction is an important determinant of finger-cuing benefits, which is in accordance with the Grouping Model.

Interestingly, for participants who started with the overlapped hands placement, the above mentioned interaction between hand placement and cue condition was present in RT, but not in pupil dilation. This dissociation between RT and pupil measures adds to previous reports that behavioral and psychophysiological measures of response preparation are not always tightly coupled (e.g., Jennings and Van der Molen, 2005). The relative insensitivity of the pupil response to cue difficulty for the overlapped-first order may be related to the fact that, overall, pupil dilation was significantly smaller for the overlapped-first order than for the adjacent-first order $(3.8 \%$ vs. $5.8 \%$, respectively). This was not due to differences in baseline pupil size values for adjacent-first and overlapped-first groups, which were very similar $\left(8.12 \mathrm{~mm}^{2}\right.$ vs. $\left.8.18 \mathrm{~mm}^{2} ; \mathrm{F}<1\right)$. Alternatively, it may reflect a strategy adopted by participants to limit the amount of invested effort when the task becomes very complex. In this view, when participants start with an extremely difficult (overlapped) response set (which confuses the normal left-to-right ordering of fingers), they are less willing to invest cognitive effort in the task than when they start with a relatively easy (adjacent) response set, and this cognitive set extends to the next to be performed condition. The finding of overall longer 
RTs and smaller pupil dilations for participants in the overlapped-first compared to the adjacent-first condition is consistent with this view. Furthermore, with relatively small amounts of invested effort, the pupil's ability to respond reliably to variations in cue difficulty may be limited.

Even though RT was not the primary dependent variable in this study, the RT data showed that, with the hands adjacent, the three informative cue conditions produced similar RTs, that is, they showed a pattern of equal RT benefits. As noted in the introduction, the typical finding in finger-cuing studies is that preparation intervals of about $3 \mathrm{~s}$ are needed to turn a pattern of differential cuing benefits into a pattern of equal cuing benefits, and the present interval of $2 \mathrm{~s}$ is substantially shorter than this $3 \mathrm{~s}$ criterion. This outcome appears to suggest that cue processing and response preparation proceeded more quickly than usually, and this may be related, at least in part, to the way in which the cues and stimuli were displayed in the present study, namely, in an overlaid arrangement and not in separate rows (underneath each other) as has typically been the case (e.g., Miller, 1982; Reeve \& Proctor, 1984). The advantage of an overlaid arrangement is that it provides a simple and stable spatial reference system (the four boxes in which cue and target stimuli appear), which may facilitate spatial processing and enhance cuing efficiency. Note that our choice for using an overlaid arrangement was motivated by the wish to minimize the occurrence of eye movements, which may interfere with the reliable measurement of pupil size.

Pupillometry proved to be extremely useful in this study because it provided information above and beyond the standard RT measure. That is, with the hands placed adjacent, the three cued conditions (left-right, inner-outer, alternate) produced similar RTs, suggesting a pattern of equal preparation difficulty. The pupil dilation data, on the other hand, showed that during the preparation interval the pupil response varied consistently with cue condition, thus revealing significant differences in cognitive processing load. Another advantage of pupillometry over RT is that the amount of cognitive effort or processing load can be estimated both before and after the moment of response initiation. Although not the focus of this study, the pupillometric waveforms depicted in Figures 3.3 and 3.4 make clear that the pupil continued to dilate following the preparation interval, reaching a maximum after the response was executed (see also Richer \& Beatty, 1985). This may suggest that monitoring processes concerned with evaluating a response carry a high cognitive load. 
In conclusion, in this study we used pupil dilation measures to augment our understanding of response preparation processes in the finger-cuing paradigm. The pupil dilation results demonstrated that left-right cues are processed most efficiently with the hands placed adjacently, but not with the hands overlapped. This outcome suggests a prominent role of motoric factors in response preparation - a role explicitly recognized by the Grouping Model of finger-cuing effects.

\section{REFERENCES}

Adam, J. J., Hommel, B., \& Umilta, C. (2003). Preparing for perception and action (I): the role of grouping in the response-cuing paradigm. Cognitive Psychology, 46(3), 302-358.

Adam, J. J., Hommel, B., \& Umiltà, C. (2005). Preparing for perception and action (II) Automatic and effortful Processes in Response cuing. Visual Cognition, 12(8), 1444-1473.

Adam, J. J., \& van Veggel, L. M. (1992). Manipulating procedural variables in a spatial precuing task. Acta Psychologica (Amst), 81(2), 97-114.

Beatty, J., \& Lucero-Wagoner, B. (2000). The Pupillary system. In J. T. Cacioppo, Tassinary, L.G. \& Berntson, GB (Ed.), Handbook of Psychophysiology (2nd ed., pp. 142-162). Cambridge: Cambridge University Press.

Brainard, D. H. (1997). The Psychophysics Toolbox. Spatial Vision, 10(4), 433-436.

Cornelissen, F. W., Peters, E. M., \& Palmer, J. (2002). The Eyelink Toolbox: eye tracking with MATLAB and the Psychophysics Toolbox. Behavior Research Methods, Instruments \& Computers, 34(4), 613-617.

Hick, W. E. (1952). On the rate of gain of information. Quarterly Journal of Experimental Psychology, 4, 11-26.

Hyman, R. (1953). Stimulus information as a determinant of reaction time. Journal of Experimental Psychology, 45(3), 188-196.

Jennings, J. R., \& van der Molen, M. W. (2005). Preparation for speeded action as a psychophysiological concept. Psychological Bulletin, 131(3), 434-459.

Jin, P. (1992). Toward a reconceptualization of the law of initial value. Psychological Bulletin, 111(1), 176-184.

Miller, J. (1982). Discrete versus continuous stage models of human information processing: in search of partial output. Journal of Experimental Psychology Human Perception and Performance, 8(2), 273-296.

Moresi, S., Adam, J. J., Rijcken, J., Van Gerven, P. W. M., Kuipers, H., \& Jolles, J. (2007). Pupil Dilation in a Response Preparation Task. International Journal of Psychophysiology, 67(2), 124-130.

Proctor, R. W., \& Reeve, T. G. (1986). Salient-Feature Coding Operations in Spatial Precuing Tasks. Journal of Experimental Psychology: Human Perception and Performance., 12(3), 277-285.

Proctor, R. W., \& Reeve, T. G. (1988). The acquisition of task-specific productions and modifications of declarative representations in spatial-precuing tasks. Journal of Experimental Psychology General, 117(2), 182-196.

Proctor, R. W., Vu, K. P., \& Pick, D. F. (2006). A deficit in older adults' effortful selection of cued responses. Journal of Motor Behavior, 38(4), 265-284.

Reeve, T. G., \& Proctor, R. W. (1984). On the advance preparation of discrete finger responses. Journal of Experimental Psychology Human Perception and Performance, 10(4), 541-553. 
Reeve, T. G., \& Proctor, R. W. (1985). Nonmotoric Translation Processes in the Preparation of Discrete Finger Responses: A Rebuttal of Miller's (1985) Analysis. Journal of Experimental Psychology: Human Perception and Performance., 11(2), 234-241.

Reeve, T. G., \& Proctor, R. W. (1990). The salient-features coding principle for spatial-and symboliccompatibility effects. In R. W. Proctor \& T. G. Reeve (Eds.), Stimulus-response compatibility (pp. 163-180). Amsterdam: North-Holland.

Reeve, T. G., Proctor, R. W., Weeks, D. J., \& Dornier, L. (1992). Salience of stimulus and response features in choice-reaction tasks. Perception and Psychophysics, 52(4), 453-460.

Requin, J. (1980). Toward a psychobiology of preparation for action. In G. E. Stelmach \& J. Requin (Eds.), Tutorials of motor behavior. (pp. 373-398). Amsterdam: North Holland.

Richer, F., \& Beatty, J. (1985). Pupillary dilations in movement preparation and execution. Psychophysiology, 22(2), 204-207.

Richer, F., Silverman, C., \& Beatty, J. (1983). Response selection and initiation in speeded reactions: a pupillometric analysis. Journal of Experimental Psychology Human Perception and Performance, 9(3), 360-370.

Rosenbaum, D. A. (1991). Human Motor Control. New York: Academic Press.

van der Molen, M. W., Boomsma, D. I., Jennings, J. R., \& Nieuwboer, R. T. (1989). Does the heart know what the eye sees? A cardiac/pupillometric analysis of motor preparation and response execution. Psychophysiology, 26(1), 70-80.

Van Gerven, P. W. M., Paas, F., Van Merriënboer, J. J. G., \& Schmidt, H. G. (2004). Memory load and the cognitive pupillary response in aging. Psychophysiology, 41, 167-174.

\section{Acknowledgements}

We would like to acknowledge Paul Willems, Anne Roefs, Huub Hamers, Jacco Ronner, Johan Gielissen, Ron Hellebrand, Michiel Vestjens, Bart Ament and Judith Helmink for providing their support to this study. 


\section{RESPONSE PREPARATION WITH \\ VALID AND INVALID CUES \\ CHAPTER 4}

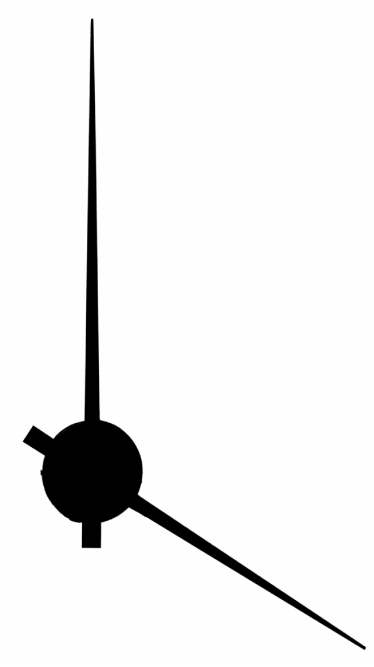

Sofie Moresi, Jos J. Adam, Jons Rijcken \& Pascal W.M. Van Gerven (2008).

Cue validity effects in Response Preparation:

a pupillometric study.

Brain Research. 1196, 94-102. 


\section{ABSTRACT}

This study examined the effects of cue validity and cue difficulty on response preparation to provide a test of the Grouping Model (Adam et al., 2003a, 2005). We used the pupillary response to index the cognitive processing load during and after the preparatory interval $(2 \mathrm{~s})$. Twenty-two participants performed the finger-cuing tasks with valid (75\%) and invalid (25\%) cues. Results showed longer reaction times, more errors, and larger pupil dilations for invalid than valid cues. During the preparation interval, pupil dilation varied systematically with cue difficulty, with easy cues (specifying 2 fingers on 1 hand) showing less pupil dilation than difficult cues (specifying 2 fingers on 2 hands). After the preparation interval, this pattern of differential pupil dilation as a function of cue difficulty reversed for invalid cues, suggesting that cues which incorrectly specified fingers on one hand required more effortful reprogramming operations than cues which incorrectly specified fingers on two hands. These outcomes were consistent with predictions derived from the Grouping Model. Finally, all participants exhibited two distinct pupil dilation strategies: an "early" strategy in which the onset of the main pupil dilation was tied to onset of the cue, and a "late" strategy in which the onset of the main pupil dilation was tied to the onset of the target. Thus, whereas the early pupil dilation strategy showed a strong dilation during the preparation interval, the late pupil strategy showed a strong constriction. Interestingly, only the late onset pupil dilation strategy revealed the above reported sensitivity to cue difficulty, showing for the first time that the wellknown pupil's sensitivity to task difficulty can also emerge when the pupil is constricting instead of dilating.

\section{INTRODUCTION}

Preparing for action typically speeds up performance. The underlying processes of response preparation have been studied by means of responsecuing paradigms, in which cues provide information about some, or all, of the required response parameters before the target stimulus appears. This line of research has started with the work of Rosenbaum (1980) who devised the movement-cuing technique to examine the preparation of aimed reaching responses. Not much later, Miller (1982) adapted this paradigm to study the preparation of discrete finger responses. In Miller's finger-cuing paradigm, 
spatial cues provide advance information about a subset of two possible finger (key press) responses out of a total of four, thus allowing the selection and preparation of a subset of finger responses.

Most finger-cuing studies have used cues that were always (i.e., 100\%) valid. In the present study, however, we lowered cue validity to $75 \%$ to investigate the effect of valid $(75 \%)$ and invalid $(25 \%)$ cues on response preparation in finger-cuing. The rationale for this manipulation of cue validity is explained later. We used the Grouping Model of finger-cuing effects, proposed by Adam et al. $(2003,2005)$, as our inferential framework. Furthermore we used reaction time (RT), error rate, and a psychophysiological measure, the pupillary response, as dependent variables.

It is well established that the cognitive pupillary response, or the task-related change in pupil size, is a reliable index of mental effort or resource allocation. The consistent finding is that more difficult tasks, which are associated with greater processing load or mental effort, evoke larger pupil dilations (for reviews, see Beatty \& Lucero-Wagoner, 2000; Jennings \& Van der Molen, 2005). This has been demonstrated in many fields of study, including perception, memory, reasoning, and attention. Recently, we have shown that pupil size also is a reliable correlate of processing load in response preparation (Moresi et al., 2008).

In the finger-cuing task participants are forewarned by a visual cue about a particular subset of possible finger responses. Typically, the cues are $100 \%$ valid so that they allow strong preparation of the cued finger responses. Horizontally arranged stimuli are reacted to by spatially compatible key press responses with the index and middle fingers of both hands placed adjacently. In the present study, the visual display consisted of four white boxes on a computer monitor, in which the cue and target signals were presented (see Figure 2.1). At the start of a trial, four white boxes appeared for $500 \mathrm{~ms}$. Then the cue signal was presented by colouring two boxes grey. After a delay of $2 \mathrm{~s}$ the target stimulus was presented by making one of the two grey boxes black; this indicated the required finger response. The $2 \mathrm{~s}$ time interval between onset of the cue and onset of the target stimulus is called the preparation interval, as it reflects the amount of time participants have to selectively prepare the cued finger responses before onset of the target stimulus.

The functional significance of the cue is that it converts the basic four-choice task into a two-choice task. Four cue or preparation conditions can be 
identified (Figure 2.1). In the hand-cued condition, the cue specifies two fingers on the same hand (e.g., the left-middle and left-index fingers). In the finger-cued condition, this cue specifies the same finger on two hands (e.g., the left-index and right-index fingers). In the neither-cued condition, the cue specifies different fingers on two hands (e.g., the left-index and right-middle fingers). These three preparation conditions are called the "cued" conditions. Also, an "uncued" control condition is included. The cue does not yield advance information about the upcoming response (all four boxes turn grey), thereby preventing selective response preparation. In other words, the uncued condition leaves the basic 4-choice task unaltered and, thus, is a control or baseline condition against which the effects of the "cued" conditions can be evaluated. Since RTs in 2-choice tasks are substantially shorter than RTs in 4-choice tasks (Hick, 1952; Hyman, 1953), cue effectiveness is inferred from a significant RT advantage or benefit (in case of valid cues) for the 2-choice "cued" conditions (i.e., hand-, finger-, and neither-cued) over the control, 4-choice (uncued) condition.

A strong and often replicated observation in the finger-cuing paradigm (with $100 \%$ valid cues) is a pattern of differential cuing benefits: RTs are shortest for the hand-cued condition, longest for the neither-cued condition, and intermediate for the finger-cued condition, reflecting an ordering in terms of preparation difficulty (for a review, see Reeve and Proctor, 1990). This pattern of differential cuing benefits, however, only appears with short preparation intervals (i.e., intervals less than about $1.5 \mathrm{~s}$ ). When the preparation interval is extended to $2 \mathrm{~s}$ or more, the three cued conditions often show comparable RTs. Thus, certain pairs of responses can be selected and prepared more quickly than others, with no differences between the pairs given sufficiently long preparation time.

A recent account of the pattern of differential cuing benefits is the Grouping Model (Adam et al., 2003a, 2005), which is an extension of the salient-features coding principle (Reeve \& Proctor, 1990). The basic idea of the Grouping Model is that the individual elements of multi-element visual displays and multi-element response arrays are not processed independently but are preattentively organized or "grouped" according to low-level grouping factors that may depend on stimulus driven factors (e.g., Gestalt principles) and on response-related factors (e.g., inter-response dependencies). According to the Grouping Model, the processing advantage of the handcued condition simply reflects the natural and stronger grouping of the two leftmost and two rightmost elements in both the stimulus and response set. In other words, each stimulus set and each response set has a default 
organization set up preattentively by the bottom-up computation of perceptual and motor units or subgroups. This process is fast and automatic. With additional, top-down processing, however, alternative organizations (finger- and neither-cued) can be achieved. This process is slow and effortful. Thus, the pattern of cuing effects that emerges in the finger-cuing task critically relies on the nature of these default groupings and on the available time to reorganize these representations, when necessary.

Assumptions about good grouping are open to independent tests. For instance, the strong grouping of left and right perceptual subgroups is bolstered by independent evidence showing that humans spontaneously and naturally divide the visual space into left-side and right-side parts (e.g., Mapp \& Ono, 1999; Nicoletti \& Umiltà, 1989). The stronger grouping of fingers on one hand as opposed to fingers on different hands is substantiated by the well-known fact that cerebral control of hand and finger movements is almost completely localized in the contralateral frontal lobe (e.g., Hellige, 1993).

According to the Grouping Model, the bilateral finger- and neither-cues are the more difficult cues because they require slow, effortful, top-down processing to break up the default, left-right spatial organization of the task environment and to create a new (perceptual and motor) organization based on the characteristics of the cue. In this view, the neither-cued condition is more difficult than the finger-cued condition, because the former represents a perceptually asymmetrical subgroup that indicates the selection and preparation of two different, non-homologous fingers on two hands, whereas the latter represents a perceptually symmetrical subgroup that specifies the same or homologous fingers on two hands. Because homologous fingers are neurally and functionally linked (Rosenbaum 1991), they are easier to group than non-homologous fingers. Manipulating perceptual and motor grouping factors, Adam et al. (2003a, 2005) showed that their independent and interactive RT effects on cuing efficiency were consistent with predictions derived from the Grouping Model.

The primary purpose of the present research was to examine the effects of cue validity on RT and pupil dilation in the finger-cuing task with the goal to provide a further test of the Grouping Model. The predictions for RT were as follows. Because the preparation interval was rather long $(2 \mathrm{~s})$, there was sufficient preparation time for all three informative cue conditions (hand-, finger-, and neither-cues) to establish a robust preparatory set, and, hence, similar RTs were expected for these informative cues. Furthermore, responses 
to valid cues should be significantly faster overall than responses to invalid cues, because invalid cues require time-consuming reprogramming or restructuring operations to counteract the "misplaced" preparatory effects caused by the invalid cue (e.g., Amrhein et al., 1991).

It should be noted, however, that even though the RTs for the three informative cue conditions might be similar, the amount of mental effort or cognitive resources invested to produce these effects might be different. According to the Grouping Model, hand-cues are "easy" cues and finger- and neither-cues are more "difficult" cues, because the selection and preparation of a subset of two fingers proceeds more efficiently when they are grouped together on one hand than when they are distributed over two hands. Hence, the processing load of hand-cues is less than that of finger- and neither-cues, with the latter, due to its asymmetric and non-homologous nature, requiring the most cognitive effort. Thus, even though these processing differences between the informative cues might not be reflected in RT with the present long (2 s) preparation interval, the Grouping Model would predict differences in pupil response as a function of preparation difficulty, reflecting underlying differences in processing load. Thus, during the preparation interval, the largest pupil dilation should be observed in the neither-cued condition, the smallest in the hand-cued condition and an intermediate level of pupil dilation should be observed in the finger-cued condition.

During the preparation interval, these predictions should hold regardless of cue validity, because the validity of the cue is determined only after this interval, that is, when the target stimulus is presented. When the target appears in one of the positions indicated by the cue, the trial represents a validly cued trial. When the target appears in one of the positions not indicated by the cue, the trial represents an invalidly cued trial. Thus, the occurrence of the stimulus determines whether a trial contains a valid or invalid cue. Consequently, we expected differences in pupil reactions for valid and invalid trials only after occurrence of the target.

On the assumption that participants prepare the responses indicated by the cue, on invalid trials the occurrence of the stimulus requires additional reprogramming or restructuring operations, possibly including suppression of already prepared responses and/or activation of already suppressed ones. Thus, after stimulus presentation, overall, larger pupil dilations are expected for invalid than valid cues. Furthermore, restructuring operations for handcues might be especially effortful because, as shown by a functional 
neuroimaging study (Adam et al., 2003), hand-cues are associated with stronger basal ganglia activity than finger- and neither-cues, suggesting stronger inhibition of irrelevant responses grouped together in one hemisphere.

An additional issue addressed in this study was that of strategies. It is well known that participants can develop specific information processing strategies based on, for example, the constraints imposed by the informational structures in the task (e.g., Logan \& Zbrodoff, 1982). It is also known that participants may shift from one strategy to another as the experiment progresses because they learn to adapt to the conditional probabilities in the task (e.g., Hunt \& Aslin, 2001). In the finger-cuing task, both the cue and the target signal carry task-relevant information, and participants may allocate cognitive resources in different degrees to both signals, especially when the cue is not always valid, as was the case in the present study. Preliminary analyses of the pupil responses indeed revealed that participants exhibited two distinct types of pupil response: An "early" strategy in which the onset of the main pupil dilation was synchronized with the onset of the cue, and a "late" strategy in which it was synchronized with the onset of the target. These two pupil response strategies are displayed in Figure 4.2. The early pupil dilation onset strategy reflects a robust and steady increase in pupil size that begins shortly after cue onset and that continues throughout the cue-stimulus (i.e., preparation) interval. The late pupil dilation onset strategy reflects a decrease in pupil size during the greater part of the cue-stimulus interval, followed by a strong increase starting shortly before the onset of the target. These two pupil response strategies were used by all participants in this study and occurred about equally often averaged over all trials (averaged across participants, $51.1 \%$ vs. $48.9 \%$ of the trials showed an early- vs. late-onset pupil dilation strategy). Thus, a second goal in this study was to examine how these two rather distinct pupil dilation strategies would affect RT and the pupillary response as a function of cue validity, cue difficulty, and blocks of trials.

\section{METHOD}

\section{Participants}

Participants were 22 right-handed students from Maastricht University (12 women, 10 men; mean age $=21.8$ years, $\mathrm{SD}=2.2$ ). All had normal or correctedto-normal vision. None of them took eye medication that could influence 
pupil responses. Informed consent was obtained and they received $€ 7.50$ for their participation.

\section{Stimuli and apparatus}

The visual display consisted of a row of four empty, white boxes $(0.5 \times 0.5 \mathrm{~cm})$ in black outline, presented on a standard computer screen with a grey background, which corresponded to the calibration background. The two center boxes were separated by $1.0 \mathrm{~cm}$, and the two left-most and two rightmost boxes were separated by $0.5 \mathrm{~cm}$ (side-to-side). Participants were seated in a height-adjustable chair with their heads stabilized in a chinrest placed at a distance of $57 \mathrm{~cm}$ from the monitor. Participants placed the index and middle fingers of both hands on four linearly arrayed push buttons mounted on a response box, which was positioned in front of them on a table and aligned with the centre of the stimulus set. The illuminance in the room was constant (about $600 \mathrm{~lx}$ ). RTs were measured in milliseconds. The software controlling the RT measurements and the stimulus presentation was programmed in Matlab 7, supported by the Eyelink Toolbox (Brainard, 1997; Cornelissen, Peters, \& Palmer, 2002) The pupil area was measured in pixels by the Eyelink I Gazetracker (SR Research, Canada), a head-mounted infrared video-based tracking system with a temporal resolution of $250 \mathrm{~Hz}$, a $0.005^{\circ}$ gaze and eye resolution, and a gaze position accuracy with a $0.5-1.0^{\circ}$ error.

\section{Task}

A trial started with the presentation of the four empty white boxes (warning signal). After a delay of $1 \mathrm{~s}$ the cue was presented by colouring two of the four boxes grey. Then, after an additional delay of $2 \mathrm{~s}$, the target stimulus was presented by colouring one of the two grey boxes black, signalling the required finger response. After each response there was an inter-trial interval of $2 \mathrm{~s}$.

\section{Design and Procedure}

Each participant received four blocks of 64 test trials (each preceded by 8 practice trials), separated by a short break of $1 \mathrm{~min}$, totalling to 256 test trials. Within the series of 256 trials, there were 64 trials for each cue condition (hand-cued, finger-cued, neither-cued, and uncued) presented in random order. In each of the informative cue conditions (hand-, finger- and neithercued), 48 trials $(75 \%)$ were valid and 16 (25\%) were invalid. Participants were informed about the nature of the task and were explicitly told to take advantage of the informative cues and to prepare the cued responses even though they were only $75 \%$ valid. They were instructed to react as quickly and 
accurately as possible to the target stimulus by pressing the correct response key. No feedback was provided. At the start of each individual test session, a nine-point calibration and validation procedure of the Eyelink I Gazetracker was performed, followed by 20 trials to familiarize participants with the task.

\section{Analyses}

Trials with RTs of less than $125 \mathrm{~ms}$ or in excess of $1250 \mathrm{~ms}(0.22 \%)$ were considered outliers and were excluded from all data analyses. Mean correct RTs and proportions of errors were calculated for each subject as a function of cue validity (valid, invalid), cue condition (hand, finger, neither, uncued), and pupil strategy (early vs. late dilation onset) and submitted to repeated measures analyses of variance (ANOVA) with the Huynh-Feldt correction applied when necessary.

Pupil data were analysed from the left eye and only for correct trials (i.e., trials without response errors and RT outliers). Eye blinks were filtered out by a computer algorithm and area samples were replaced by cubic spline interpolation, starting 17 samples $(68 \mathrm{~ms})$ before and ending 25 samples (100 ms) after the blink ( $\pm 10 \%$ drop out). For each trial, a baseline pupil area was determined by calculating the average pupil size during a period of $500 \mathrm{~ms}$ preceding the onset of the cue signal. Cue-locked pupil dilation or constriction was calculated by subtracting this baseline area from the pupil area during a period of $4 \mathrm{~s}$ following cue onset. This difference score was converted to a percentage of the corresponding baseline value. This conversion to percentages was warranted by Jin's (1992) revised version of the so-called Law of Initial Value (LIV), which states that within the middle range of baseline values the physiological response increases as a function of baseline. This version of the LIV was applicable to our data, because there was a positive overall correlation between baseline pupil size and peak dilation ( $r$ $=.50)$. The LIV introduces a physiological artefact, however, because it influences pupil dilation and thus may obscure the effect of cue condition. Expressing dilation as a percentage of the baseline corrects for this artefact, because pupillary responses following a relatively high baseline value are attenuated.

Pupil strategy was determined by comparing the pupil dilation at cue onset with the pupil dilation at $90 \%$ of the preparation interval: If the pupil size at $90 \%$ preparation interval was still less than that at cue onset, the trial was classified as reflecting a "late" dilation strategy; otherwise it was classified as reflecting an "early" dilation strategy. 


\section{RESULTS}

\section{Behavioral data}

\section{Reaction Time}

Mean RT as a function of cue validity and cue condition is shown in Figure 4.1A. A repeated measures analysis of variance (ANOVA) was performed on mean RT with cue validity (valid, invalid), cue condition (hand-, finger-, neither-cued), and pupil strategy (early vs. late dilation onset) as withinsubject variables. There was only one significant effect, namely the main effect of cue validity $F(1,21)=147.16, p<.001$, indicating substantially longer RTs for invalid than for valid cues (means $=464 \mathrm{~ms}$ vs. $369 \mathrm{~ms}$, respectively). This cue validity effect was independent of cue condition $(p>.25)$. Furthermore, all F-values involving the factor pupil strategy were nonsignificant (all ps $>.27$ ), indicating that pupil strategy did not influence RT. Mean RT for the uncued condition ( $414 \mathrm{~ms}$ ) was intermediate to those of valid $(369 \mathrm{~m})$ and invalid cues $(464 \mathrm{~ms})$, demonstrating significant $(\mathrm{p}<.001)$ benefits and costs for valid and invalid cues, respectively (see Figure 4.1A).

Errors

The overall mean error rate was $2.5 \%$. Mean error rate as a function of cue validity and cue condition is shown in Figure 4.1B. We conducted a repeated measures analysis of variance (ANOVA) on the arcsine transforms of the error rates (to attain homogeneity of error variance; Keppel, 1982) with cue validity (valid, invalid), cue condition (hand-, finger-, neither-cued), and pupil strategy (early vs. late dilation onset) as within-subject variables. This analysis revealed that error rates tended to be greater for invalid than valid trials $(3.8 \%$ vs. $2.0 \%$, respectively; $F(1,21)=4.07, p=.057)$. Furthermore, as shown in Figure 4.1B, this cue validity effect for errors was greatest for hand-cues $(4.3 \%$ vs. $1.0 \%$, respectively; $p<.05)$, intermediate for finger-cues ( $4.6 \%$ vs. $2.2 \%$, respectively; $p<.05)$, and absent for neither-cues $(2.4 \%$ vs. $2.6 \%$, respectively; $p>0.5)$, as revealed by an interaction between cue validity and cue condition that approached significance, $F(2,42)=2.56, p=.090$. There was no effect of pupil strategy (all ps $>$.3). The neutral cue condition produced an error rate of $1.9 \%$. 

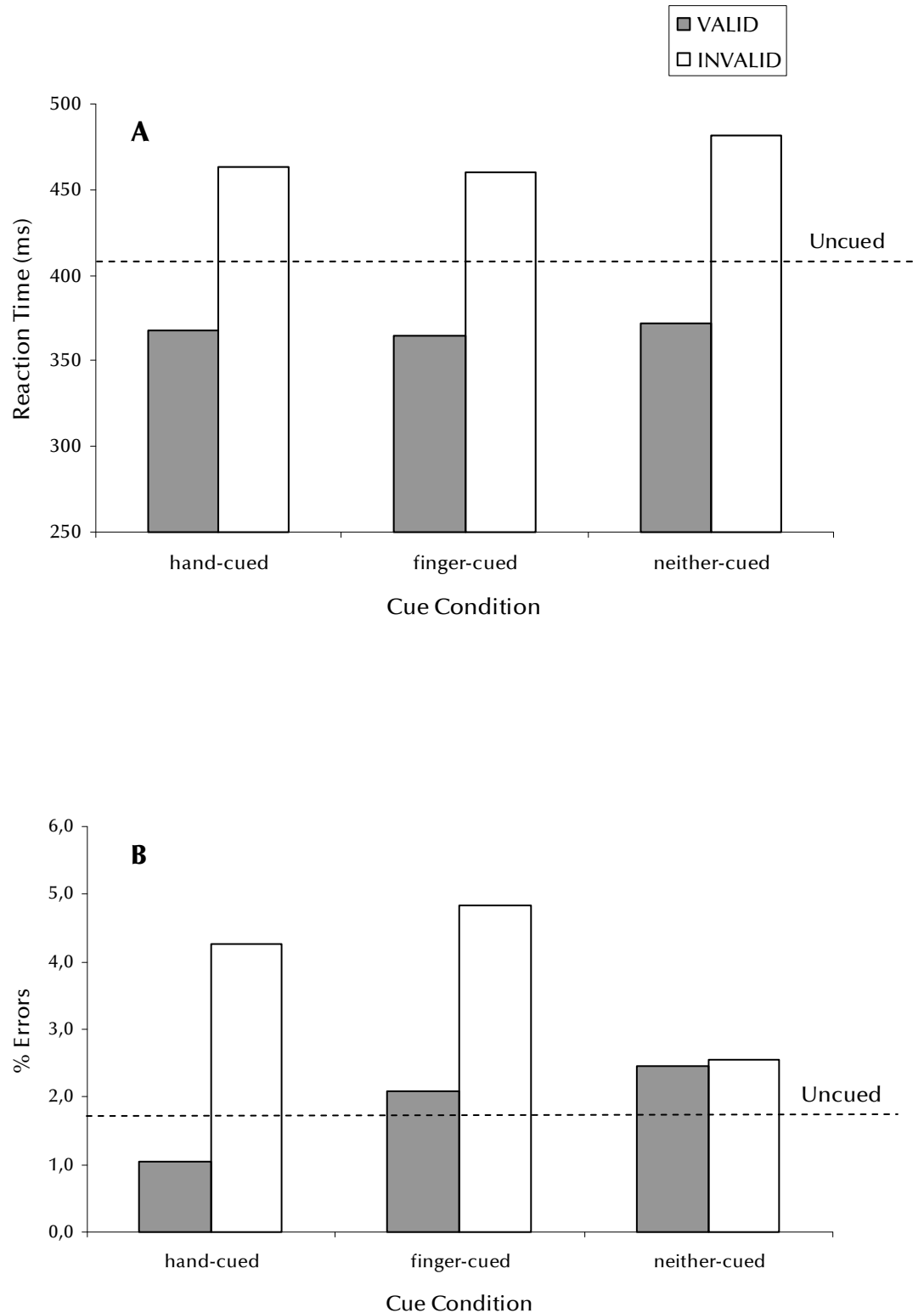

Figure 4.1. (A) Mean reaction time (ms) and (B) mean percentage of errors as a function of cue validity and cue condition. 


\section{Pupil data}

Mean percentage pupil dilation across all participants is plotted in Figure 4.2 as a function of time (starting $500 \mathrm{~ms}$ before cue onset), cue condition, cue validity, and pupil dilation onset strategy. To quantify the pupil response we calculated mean pupil dilation as a function of cue condition, cue validity, and pupil strategy for two time periods: the 500-2000 ms time interval and the 2000-3500 ms time interval after cue onset, respectively as an indicator of cognitive load during and after the preparation interval. The resulting mean pupil data were entered in separate 2 (cue validity) x 3 (cue condition) repeated measures ANOVAs for the time periods during and after the preparation interval and for each of the two pupil strategies. It should be noted that negative pupil dilation values indicate a smaller pupil size relative to the baseline value, thus reflecting a process of pupil constriction. Hence, differences in degree of relative constriction should be interpreted as differences in dilation.

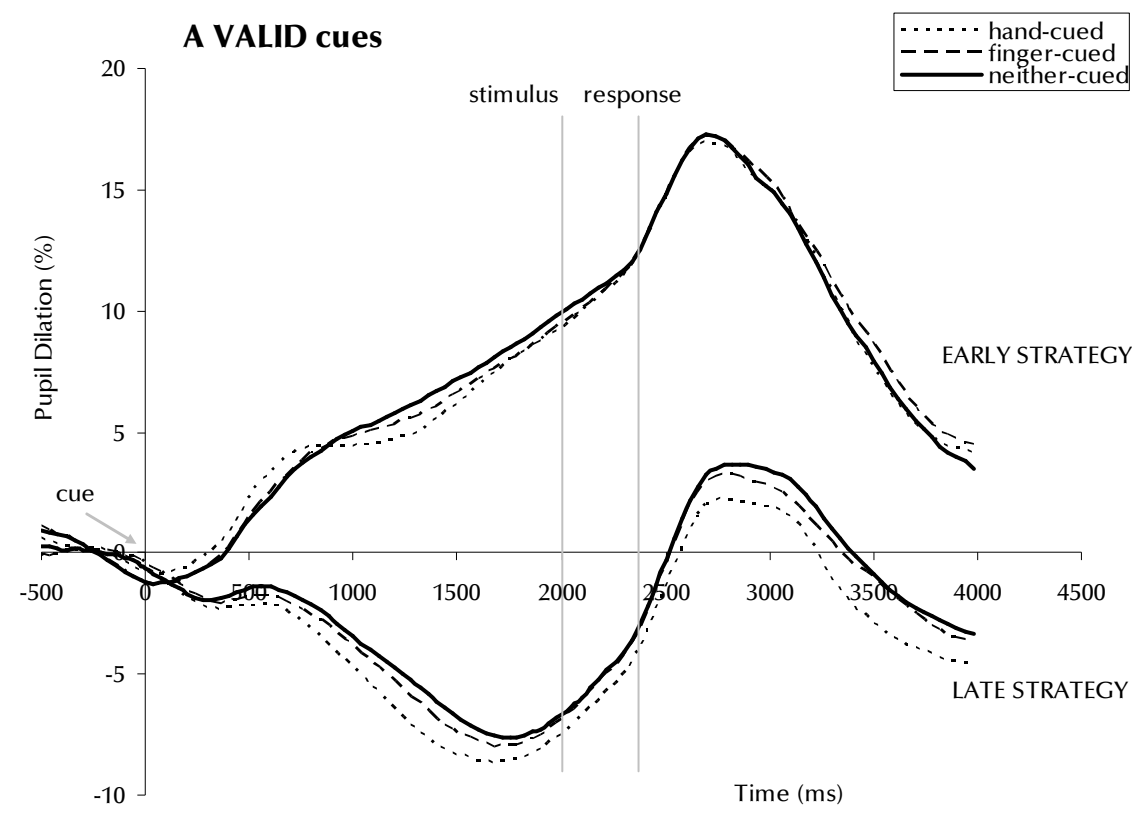




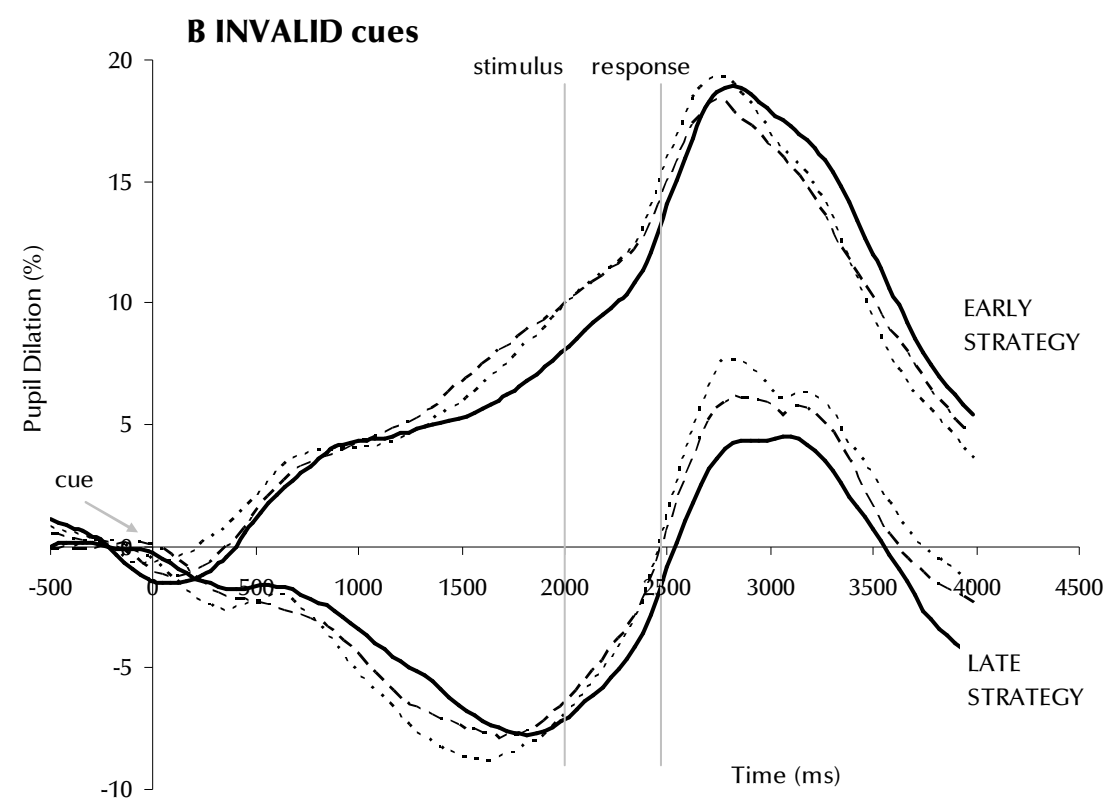

Figure 4.2. The dilation-by-time plots for (A) valid and (B) invalid cues for the three cued conditions and two pupil dilation strategies, starting from cue onset until $4 \mathrm{~s}$ afterwards. The stimulus is presented $2 \mathrm{~s}$ after cue onset, and the response occurs, on average, $369 \mathrm{~ms}$ and $464 \mathrm{~ms}$ after stimulus onset for valid and invalid cues, respectively.

As predicted, pupil dilation was not different for valid and invalid cues during the preparation interval, $F(1,21)=2.50, \mathrm{p}>0.12$, but after stimulus presentation pupil dilation was significantly greater for invalid than valid cues, $\mathrm{F}(1,21)=7.11, \mathrm{p}<.05$. Interestingly, and in contrast to our expectations, there was no reliable effect of cue condition on pupil dilation both during and after the preparation interval (all ps $>.5$ ). These effects are summarized in Figure 4.3 and 4.4 . 
Late Pupil Dilation Onset

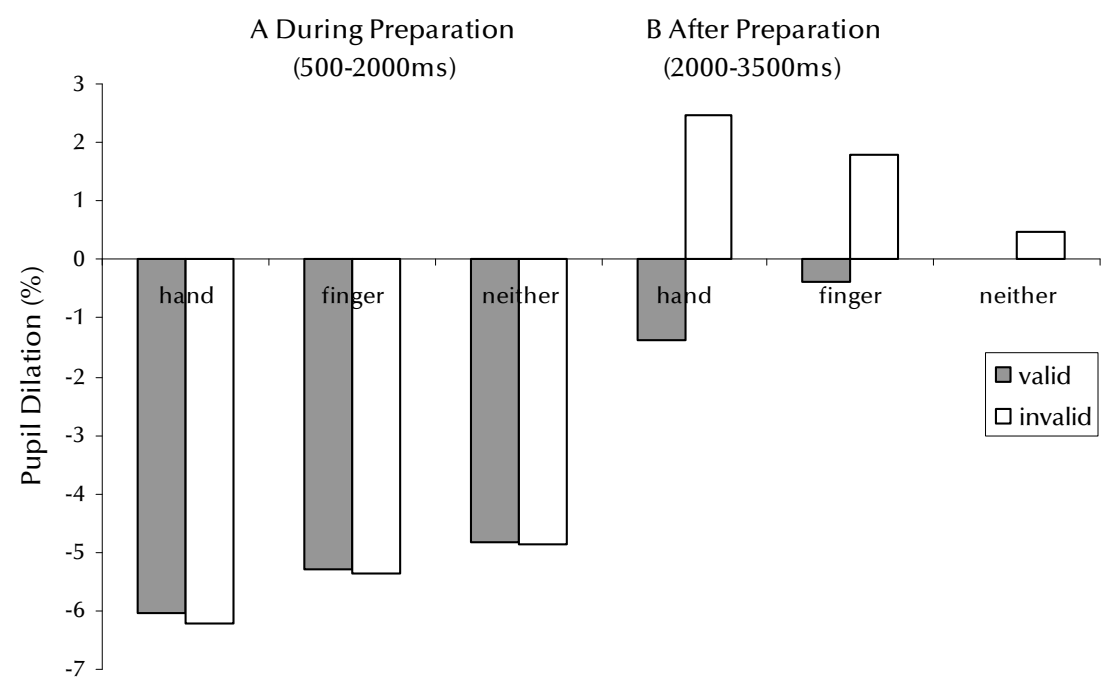

Cue Condition

Figure 4.3. Mean pupil dilation (\%) for late pupil dilation trials as a function of cue condition and cue validity (a) during and (b) after the preparation interval.

Late pupil dilation onset trials

As expected, valid and invalid cues did not generate different pupil dilations during the preparation interval, $F(1,21)<1$, but they did so after stimulus presentation, with larger pupil dilations for invalid than valid cues, $F(1,21)=$ 23.73, $\mathrm{p}<.001$ (see Figure 4.3). Importantly, and in line with the predictions derived from the Grouping Model, during the preparation interval, more difficult cues were associated with greater pupil dilations, $F(2,42)=7.64, p<$ .001 (see Figure 4.3). After the preparation interval, there was a significant interaction between cue condition and cue validity, $\mathrm{F}(2,42)=3.42, \mathrm{p}<.05$ (see Figure 4.3). This interaction indicates that the increase in pupil size for invalid (relative to valid) cues was strongest for hand-cues $p<.001$, intermediate for finger-cues $p<.05$, and least (non-significant) for neither-cues $p>.5$. Note that this specific ordering of pupil size as a function of cue condition for invalid cues after the preparation interval was opposite to the pattern observed during the preparation interval. This finding was statistically confirmed by a 3 (cue condition) $\times 2$ (time interval) ANOVA on the pupil data of the invalid cues, which yielded a significant interaction, $F(2,42)=5.21, p<$ .02 . Thus, with invalid cues, hand-cues showed the least and neither-cues the 
greatest pupil dilation during the preparation interval, but after the presentation of the stimulus this order reversed with larger pupil dilations for hand-cues than for neither-cues (see also Figure 4.2B).

\section{Early Pupil Dilation Onset}

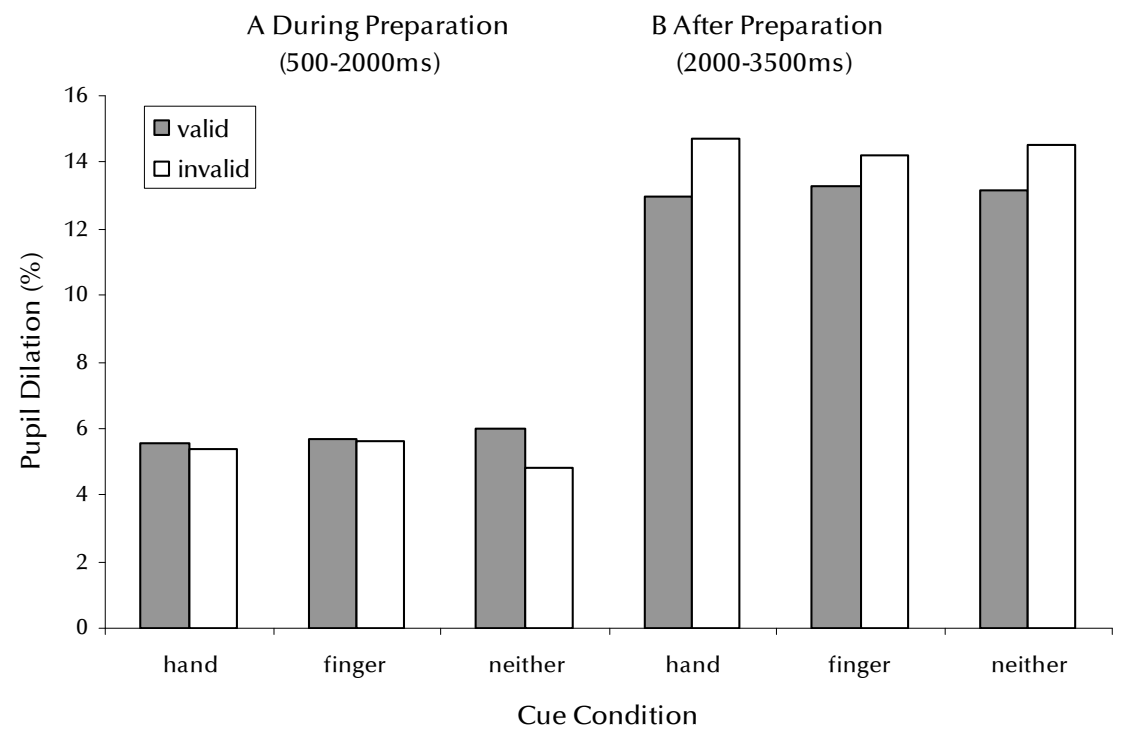

Figure 4.4. Mean pupil dilation (\%) for early pupil dilation trials as a function of cue condition and cue validity (a) during and (b) after the preparation interval.

Early versus late pupil dilation onset strategy

As noted in the introduction, the relative occurrence of the early versus late dilation onset strategy was about equal averaged over all trials and all participants $(51.1 \%$ and $48.9 \%$, respectively, standard deviation $=10.3 \%)$. Importantly, all participants showed these two strategies with the largest difference between these two strategies for an individual participant being $35 \%$ vs. $65 \%$. Also, the (early) strategy appeared to be independent of cue condition $\mathrm{F}(3,63)<1$ (means $=47.8 \%, 51.3 \%, 54.1 \%$, and $52.8 \%$ for the neutral, hand-, finger-, and neither-cued conditions, respectively) and cue validity $\mathrm{F}(1$, 21 ) $<1$ (means $=52.9 \%$ and $52.6 \%$ for valid and invalid cues, respectively).

To get some insight in the distribution of these two strategies over the time course of the full experiment (consisting of four blocks of each 64 experimental trials), we calculated the percentages of trials with an early 
dilation strategy as a function of 8 consecutive trials. That is, we sorted the total of 256 trials into 32 consecutive bins (each containing eight trials), and calculated for each participant the percentages of trials with an early strategy as a function of bin. The results are plotted in Figure 4.5. Visual inspection reveals that early in a block of 64 trials the early dilation strategy tended to occur more often than the late strategy, but as the block progressed this pattern reversed, with the late dilation strategy occurring more often than the early strategy. This pattern was evident in all four blocks of trials, but was most pronounced in the first block. A 4 (block) x 8 (bin) repeated measures ANOVA confirmed this pattern by revealing a main effect of bin $F(7,147)=$ $8.03, \mathrm{p}<.001$ (means $=63.5 \%, 55.0 \%, 53.1 \%, 49.9 \%, 46.6 \%, 46.7 \%, 48.2 \%, 48.3 \%)$, which was qualified by an interaction with block, $F(21,441)=2.28, p<.05$.

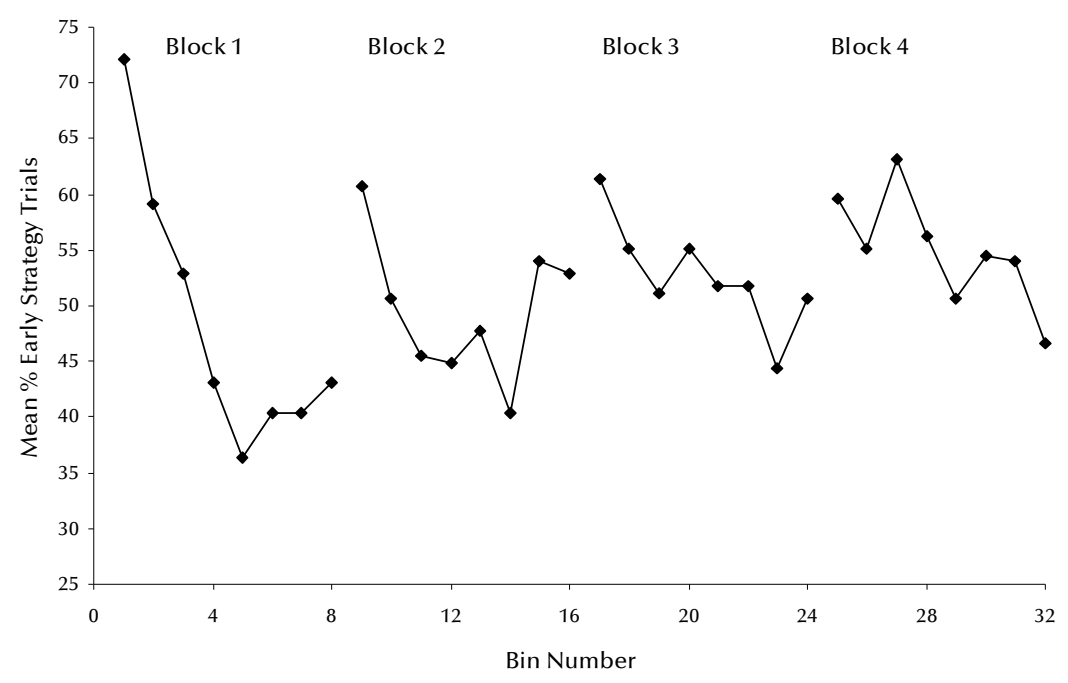

Figure 4.5. Mean percentage of trials that showed an early dilation strategy as a function of bin (which contained 8 consecutive trials) across the four blocks of each 64 trials.

\section{DISCUSSION}

In this study we examined the variation in pupil response during and after a preparatory interval of $2 \mathrm{~s}$, in which cues correctly (75\%) or incorrectly $(25 \%)$ specified a subset of two fingers to be prepared on one or two hands. As expected, the RT and error data demonstrated benefits for valid cues and costs for invalid cues. This outcome replicates previous findings (e.g., Adam \& 
Pratt, 2004; Miller, 1982), and demonstrates that participants did use the information provided by the cues and prepared the cued finger responses. The unique contribution of the present study is that we recorded the pupillary response, a psychophysiological estimate of processing load, both during and after the preparation interval, to provide insight into the dynamics of response preparation, and, furthermore, to test predictions derived from the Grouping Model.

A key finding was that, after the preparation interval, invalid cues generated larger pupil dilations than did valid cues. This outcome suggests that invalid cues imposed a greater cognitive processing load than did valid cues probably because of (re)programming or restructuring operations concerned with correcting the inappropriate, "non-functional" preparatory effects due to the invalid cue during the preparation interval.

According to the Grouping Model, cues specifying two fingers on one hand are processed more efficiently, and thus require less cognitive effort, than cues specifying two fingers on two hands. This is based on the assumption that the activation and inhibition of, respectively, relevant and irrelevant responses, is easier to implement when the relevant and irrelevant responses are represented in separate hemispheres than when they are distributed over neighbouring areas in two hemispheres. Consistent with this view, the pupil data of trials showing a late pupil dilation onset strategy showed smaller pupil dilations for less difficult cues during the preparation interval. This pattern, however, reversed for invalid cues after the preparation interval. Thus, the easiest to prepare hand-cues showed the least pupil dilation during the preparation interval, but the greatest pupil dilation after the preparation interval when a not-cued response had to be selected. On the other hand, the most difficult to prepare neither-cues showed the greatest pupil dilation during the preparation interval, but the smallest pupil dilation after the preparation interval. In fact, the increase in pupil dilation for the neither-cued condition for invalid cues relative to valid cues after the preparation interval was very small and non-significant (see Figure 4.3). This suggests a limited influence of effortful restructuring operations for invalid neither-cues.

These outcomes are consistent with the fact that hand-cues are associated with stronger basal ganglia activity than finger- and neither-cues (Adam et al., 2003b), suggesting stronger inhibition of the non-cued responses grouped together in one hemisphere. By this view, the selection of a strongly inhibited response is more effortful than the selection of a not, or less, inhibited 
response. The error data provided converging evidence for this view, because invalid cues increased error rate of hand-cues but not of neither-cues (see Figure 4.1B).

Taken together, the present data further strengthen one of the key assumptions of the Grouping Model, namely, that unilateral hand-cues are processed more efficiently than bilateral finger- and neither-cues. Hence, the present findings add to previous results indicating a strong dissociation between the two types of cue: (1) hand-cues but not finger-cues are sensitive to temporal and spatial characteristics of the cue (i.e., onset vs. no-onset cues, spatial vs. symbolic cues; Adam et al., 2005); (2) finger- and neither-cues but not hand-cues show distinct developmental effects between the ages of 7 and 21 years (Adam et al., 2006); and (3) hand-cues but not finger- and neither-cues are immune against the negative effects of aging (Adam et al., 1998; but see Proctor et al., 2006).

A striking finding was that all participants in this study used two pupil dilation strategies across trials: an "early" strategy, characterized by an increase in pupil dilation that started soon after cue presentation, and a "late" strategy, characterized by an initial decrease in pupil dilation at cue onset followed by an increase that was linked with the onset of the stimulus. The early strategy appeared to be dominant in the earlier parts of a block of trials, but, as the block progressed, the late strategy tended to become dominant. This gradual shift in relative occurrence of the two pupil strategies as a function of number of trials suggests a strategic adaptation to the occurrence of invalid cues. Interestingly, these two distinct pupil dilation strategies were not associated with reliable differences in RT and error rate. This outcome corroborates previous evidence indicating dissociation between behavioural and psychophysiological measures of response preparation (e.g., Jennings \& Van der Molen, 2005; Miller et al., 1996).

Even though both pupil strategies showed a consistent sensitivity to cue validity (i.e., larger pupil dilations for invalid than valid cues), only the "late" strategy showed the above discussed sensitivity to cue difficulty. This latter finding is of particular importance because it demonstrates, as far as we know for the first time, that the well-known sensitivity of the pupil to task difficulty can also be found when the pupil is constricting instead of dilating. Furthermore, the early strategy's insensitivity to cue difficulty could be related to its early "full-out" dilation, which may leave little room for variation in pupil dilation as a function of cue difficulty. 
Finally, it is interesting to note that, even though the two pupil dilation strategies showed opposite patterns of dilation and constriction during the preparation interval, after the preparation interval they both showed a similar pattern of strong dilation, reaching maximal dilation some time after the occurrence of the response. Thus, regardless of the actual pupil behaviour during the preparation interval, the consistent factor across the two pupil strategies was the trend to synchronize maximal dilation with response execution and response monitoring processes, as has been observed before (e.g., Richer et al., 1983).

It is relevant to discuss two alternative explanations of the hand-advantage. According to the "spatial proximity" hypothesis, preparation for two stimulus positions is more efficient the closer together they are, possibly because of an advantage in sharing attention across nearby positions (Miller, 1982). This hypothesis would predict shorter RTs when the index fingers are cued than when the middle fingers are cued, simply because the cued locations are in closer proximity in the former situation than in the latter. The data, however, contradict this prediction, because they show an opposite pattern: RTs were shorter, not longer, for preparing two middle as compared to two index fingers $($ means $=357 \mathrm{~ms} v \mathrm{~s} .372 \mathrm{~ms}$, respectively $\mathrm{t}(21)=2.68, \mathrm{p}<.05)$.

A second alternative explanation of the advantage for hand-cues focuses on the left-right distinction in the stimulus-response displays as being critical, with the hand-distinction being of little importance (e.g., Reeve \& Proctor, 1984, 1990). Experiments, however, that used a one-hand response set (Adam et al., 2003, Experiment 1; Proctor \& Reeve, 1986, Experiment 2) or an overlapped-finger response set (Adam et al., 2003, Experiment 5; but see, Reeve \& Proctor, 1984) demonstrated that the anatomical hand distinction is a prerequisite for the hand-advantage to materialize (for a more detailed discussion of this matter, see Adam et al., 2003a; Experiment 5).

\section{CONCLUSION}

In this study we used pupil dilation to better understand the cognitive brain mechanisms associated with valid and invalid response preparation. The results provide converging evidence for the Grouping Model of finger-cuing effects by demonstrating that pupil size, which indexes cognitive processing load, varies systematically with finger preparation difficulty, both during and 
after the preparation interval. During the preparation interval, easy-to-prepare hand-cues showed less pupil dilation than difficult-to-prepare finger- and neither-cues, but this pattern reversed after the preparation interval when an invalid cue required the selection of a non-cued finger. This finding suggests that invalid hand-cues require more effortful reprogramming operations than do invalid finger- and neither-cues in order to switch from the cued finger responses to one of the alternative non-cued, and possibly inhibited, ones. These findings were manifest only for trials that showed a late pupil dilation onset strategy. The functional significance of the early and late pupil onset strategies is yet unclear and requires further systematic investigation.

\section{REFERENCES}

Adam, J. J., Backes, W., Rijcken, J., Hofman, P., Kuipers, H., \& Jolles, J. (2003b). Rapid visuomotor preparation in the human brain: a functional MRI study. Cognitive Brain Research, 16(1), 1-10.

Adam, J. J., Hommel, B., \& Umiltà, C. (2003a). Preparing for perception and action (I): the role of grouping in the response-cuing paradigm. Cognitive Psychology, 46(3), 302-358.

Adam, J. J., Hommel, B., \& Umiltà, C. (2005). Preparing for perception and action (II) Automatic and effortful Processes in Response cuing. Visual Cognition, 12(8), 1444-1473.

Adam, J. J., Paas, F. G., Teeken, J. C., van Loon, E. M., van Boxtel, M. P., Houx, P. J., et al. (1998). Effects of age on performance in a finger-precuing task. Journal of Experimental Psychology Human Perception and Performance, 24(3), 870-883.

Adam, J. J., Parthoens, S., \& Pratt, J. (2006). Distinct mechanisms for planning keypress and reaching responses: a developmental study. Human Movement Science, 25(3), 293-309.

Adam, J. J., \& Pratt, J. (2004). Dissociating visual attention and effector selection in spatial precuing tasks. Journal of Experimental Psychology Human Perception and Performance, 30(6), 10921106.

Amrhein, P. C., Stelmach, G. E., \& Goggin, N. L. (1991). Age differences in the maintenance and restructuring of movement preparation. Psychological Aging, 6(3), 451-466.

Beatty, J., \& Lucero-Wagoner, B. (2000). The Pupillary system. In J. T. Cacioppo, Tassinary, L.G. \& Berntson, GB (Ed.), Handbook of Psychophysiology (2nd ed., Vol. 2nd edition, pp. 142-162). United States of America: Cambridge University Press.

Brainard, D. H. (1997). The Psychophysics Toolbox. Spatial Vision, 10(4), 433-436.

Cornelissen, F. W., Peters, E. M., \& Palmer, J. (2002). The Eyelink Toolbox: eye tracking with MATLAB and the Psychophysics Toolbox. Behavior Research Methods, Instruments \& Computers, 34(4), 613-617.

Hellige, J. B. (1993). Hemispheric Asymetry: What's Right and What's Left. Cambridge, Massachusetts. London, England.: Harvard University Press. .

Hick, W. E. (1952). On the rate of gain of information. Quarterly Journal of Experimental Psychology, 4, 11-26.

Hunt, R. H., \& Aslin, R. N. (2001). Statistical learning in a serial reaction time task: access to separable statistical cues by individual learners. Journal of Experimental Psychology General, 130(4), 658-680.

Hyman, R. (1953). Stimulus information as a determinant of reaction time. Journal of Experimental Psychology, 45(3), 188-196. 
Jennings, J. R., \& Van der Molen, M. W. (2005). Preparation for speeded action as a psychophysiological concept. Psychological Bulletin, 131(3), 434-459.

Jin, P. (1992). Toward a reconceptualization of the law of initial value. Psychological Bulletin, 111(1), 176-184.

Keppel, G. (1982). Design and Analysis: A Researcher's Handbook (second ed.). New Jersey: Prentice-Hall Inc.

Logan, G. D., \& Zbrodoff, N. J. (1982). Constraints on strategy construction in a speeded discrimination task. Journal of Experimental Psychology Human Perception and Performance, 8(4), 502-520.

Mapp, A. P., \& Ono, H. (1999). Wondering about the wandering cyclopean eye. Vision Research, 39(14), 2381-2386.

Miller, J. (1982). Discrete versus continuous stage models of human information processing: in search of partial output. Journal of Experimental Psychology Human Perception and Performance, 8(2), 273-296.

Miller, J., Coles, M. G., \& Chakraborty, S. (1996). Dissociation between behavioral and psychophysiological measures of response preparation. Acta Psychologica (Amst), 94(2), 189208.

Moresi, S., Adam, J. J., Rijcken, J., P.W.M., V. G., Kuipers, H., \& Jolles, J. (2008). Pupil Dilation in a Response Preparation Task. International Journal of Psychophysiology, 67(2), 124-130.

Nicoletti, R., \& Umilta, C. (1989). Splitting visual space with attention. Journal of Experimental Psychology Human Perception and Performance, 15(1), 164-169.

Proctor, R. W., \& Reeve, T. G. (1986). A caution regarding use of the hint procedure to determine whether partial stimulus information activates responses. Perception and Psychophysics, 40(2), 110-118.

Proctor, R. W., Vu, K. P., \& Pick, D. F. (2006). A deficit in older adults' effortful selection of cued responses. Journal of Motor Behavior, 38(4), 265-284.

Reeve, T. G., \& Proctor, R. W. (1984). On the advance preparation of discrete finger responses. Journal of Experimental Psychology Human Perception and Performance, 10(4), 541-553.

Reeve, T. G., \& Proctor, R. W. (1990). The salient-features coding principle for spatial-and symboliccompatibility effects. In R. W. Proctor \& T. G. Reeve (Eds.), Stimulus-response compatibility (pp. 163-180). Amsterdam: North-Holland.

Richer, F., Silverman, C., \& Beatty, J. (1983). Response selection and initiation in speeded reactions: a pupillometric analysis. Journal of Experimental Psychology Human Perception and Performance, 9(3), 360-370.

Rosenbaum, D. A. (1980). Human movement initiation: specification of arm, direction, and extent. Journal of Experimental Psychology General, 109(4), 444-474.

Rosenbaum, D. A. (1991). Human Motor Control. New York: Academic Press.

\section{Acknowledgements}

We would like to acknowledge Bart Ament and Judith Helmink for providing their support to this study. 


\section{RESPONSE PREPARATION \\ WITH STATIC VERSUS MOVING HANDS \\ CHAPTER 5}

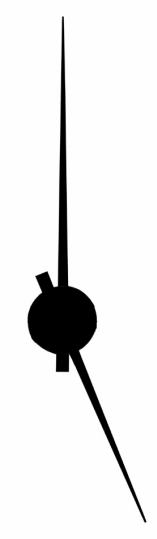

Jos J. Adam \& Sofie Moresi (2007).

Response Preparation with static versus moving hands.

Brain and Cognition, 65(3), 253-259. 


\section{ABSTRACT}

This research tested the response inhibition account of the hand-advantage found in the finger precuing task. According to this account, the advantage of preparing two fingers on one hand (represented in one hemisphere) as opposed to preparing two fingers on two hands (represented in two hemispheres) is due, in part, to a response inhibition process that operates more efficiently within than between hemispheres. In this view, supplying extra activation to both hemispheres by moving the hands should decrease the within-hemisphere inhibition advantage. Twelve participants performed the finger precuing task with static and moving hands. As predicted by the response inhibition account, the hand-advantage, present with the hands at rest, decreased with the hands moving.

\section{INTRODUCTION}

Since the pioneering work of Leonard (1958) it is well established that advance information regarding a to-be-performed action improves reaction time (RT). This beneficial effect of action preparation on RT has been studied extensively by means of the finger-cuing paradigm developed by Miller (1982), who adapted Rosenbaum's (1980) movement precuing technique. In the finger-cuing task, advance information delineates a subset of two out of four possible finger responses (usually keypress responses made by the index and middle fingers of both hands), allowing a process of selective response preparation. In this study we explore whether moving the hands would influence the response preparation effects on RT that are typically found in the finger-cuing task with the hands stationary. Before explaining the rationale of the "moving hands" manipulation, we briefly introduce the methodology of the finger-cuing task, its basic findings, and a theoretical framework for interpretation.

In the finger-cuing task, the visual display consists of three horizontal rows of symbols, representing warning, cue, and target stimulus, respectively (see Figure 1.1). The warning stimulus consists of four plus signs, indicating the four possible stimulus-response locations. The cue, that follows after a fixed 
delay, consists of two plus signs, indicating a subset of two possible stimulusresponse locations. After a certain preparation interval, that may vary between $100 \mathrm{~ms}$ and $3 \mathrm{~s}$, the target stimulus is presented, a single plus sign that indicates the required response.

The function of the cue is to provide advance information about a subset of two possible responses. That is, the cue functionally transforms the original four-choice reaction task into a two-choice reaction task. Four cue or preparation conditions are distinguished. In the hand-cued condition, the cue specifies two fingers on the same hand (e.g., the left-index finger and the leftmiddle finger). In the finger-cued condition, the cue specifies the same fingers on different hands (e.g., the two index fingers). In the neither-cued condition, the cue specifies different fingers on different hands (e.g., the leftmiddle and right-index fingers). Also, an uncued condition is included, which provides no advance information, and thus precludes selective preparation of any combination of two finger responses. This condition is a necessary control condition because it leaves the basic, four-choice task unaltered. Since two-choice responses normally yield shorter RTs than do four-choice responses (Hick, 1952; Hyman, 1953), cue effectiveness is inferred from a significant RT advantage for the two-choice cue conditions (i.e., hand-cued, finger-cued, and neither-cued) over the control, four-choice (uncued) condition.

The robust finding from the finger-cuing paradigm is a pattern of differential cuing benefits: RTs are shortest for the hand-cued condition and longest for the neither-cued condition, with RTs for the finger-cued condition being intermediate (Reeve \& Proctor, 1984, 1990; Proctor \& Reeve, 1986, 1988). This pattern of differential cuing benefits is apparent primarily at short preparation intervals (intervals shorter than 1,500 ms), with longer preparation intervals producing a pattern of equivalent cuing benefits. Thus, with sufficient preparation time, all possible pairs of finger responses can be selected and prepared to the same degree (Reeve \& Proctor, 1984, 1990; Proctor \& Reeve, 1986, 1988).

A recent account of the pattern of differential cuing benefits is the Grouping Model (Adam, Hommel, \& Umiltà, 2003, 2005), that is an extension of the salient-features coding principle of Proctor and Reeve (1986, 1988; Reeve \& Proctor, 1990). The key idea of the Grouping Model is that the individual elements of multielement visual displays and multielement response arrays are not processed independently but are preattentively organized or 
"grouped" according to low-level grouping factors that may depend on stimulus driven factors (e.g., Gestalt principles) and on response-related factors (e.g., interresponse tendencies). According to the Grouping Model, the processing advantage of the hand-cued condition simply reflects the natural and stronger grouping of the two leftmost and two rightmost elements in both the stimulus display and the response array. In other words, each stimulus set and each response set has a default organization established preattentively by the bottom-up computation of perceptual and motoric units or subgroups; this process is fast and automatic. With additional, top-down processing, however, alternative organizations can be attained; this process is slow and effortful. Thus, the pattern of cuing effects that emerge in the finger cuing task critically depends on the nature of these default groupings and on the time available to reorganize these representations, if necessary. According to the Grouping Model, finger- and neither-cues are the more difficult cues because they require slow, effortful, top-down processing to breakup the default, left-right spatial organization and to create a new organization based on the characteristics of the cue. Manipulating perceptual and motoric grouping factors, Adam et al. (2003, 2005) showed that their independent and interactive effects on cuing efficiency were consistent with predictions derived from the Grouping Model.

To determine the neural mechanisms of the RT advantage for hand-cues, Adam, Backes, Rijcken, Hofman, Kuipers, and Jolles (2003) conducted an fMRI study of the neuronal activation patterns in finger-cuing. Their imaging results revealed a single but distributed network of neural areas involved in finger preparation, including specific areas of the frontal cortex, the parietal cortex, and basal ganglia. Importantly, this system modulated activity in the parietal cortex and basal ganglia depending on the type of cue. In particular, it was found that hand-cues reduced activity in the parietal cortex but increased activity in the basal ganglia (relative to finger-and neither-cues). ${ }^{1}$ Hence, the hand-advantage was shown to have two neural components.

The decreased activity in the parietal cortex, known to derive multiple representations of space to guide different kinds of action (e.g., Colby \& Goldberg, 1999), is consistent with the Grouping Model's assumption that hand-cues represent strong, natural subgroups that are established quickly and automatically, requiring less processing activity than the more difficult finger- and neither-cues. The increased activity in the basal ganglia is more difficult to interpret but given the evidence that, physiologically, the output 
from the basal ganglia is inhibitory, it could be understood in terms of a fast, robust inhibition process targeted at one hemisphere, that is, the hemisphere representing the two irrelevant responses. In this view, selecting and preparing motor responses does not only implicate selection and activation of the relevant ones but also inhibition or suppression of the irrelevant, nondesirable ones (e.g., Mink, 1996). The notion of response inhibition as a functional counterpart of response activation is supported by empirical evidence (e.g., Burle, Vidal, Tandonnet, \& Hasbroucq, 2004; Jennings \& Van der Molen, 2005), and often used as a compelling theoretical construct in models of choice RT (e.g., Kornblum, Hasbroucq, Osman, 1990; Usher \& McClelland, 2001). Adam et al. (2003) speculated that inhibition of the (pre)motor cortex of one hemisphere (in case of hand-cues) is easier to implement than the more specific inhibition of two irrelevant responses in two hemispheres (in case of finger- and neither-cues). This is because in the latter case, relevant and irrelevant responses are represented by neighboring or bordering neural areas in two hemispheres, necessitating both a finer and more distributed spatial inhibition process than when the relevant and irrelevant responses are located in separate hemispheres, which allows a fast, robust and rather global process of response inhibition concentrated in one hemisphere.

The aim of the present research was to provide a test of this response inhibition account of the hand-advantage observed in the finger cuing task. The key experimental manipulation consisted of "moving the hands" during finger-cuing task performance. That is, we compared finger-cuing task performance in a "dynamic" condition, where participants moved their two hands continuously back and forth, against the standard "static" condition, where participants did not move their hands. Figure 5.1 illustrates the apparatus. 


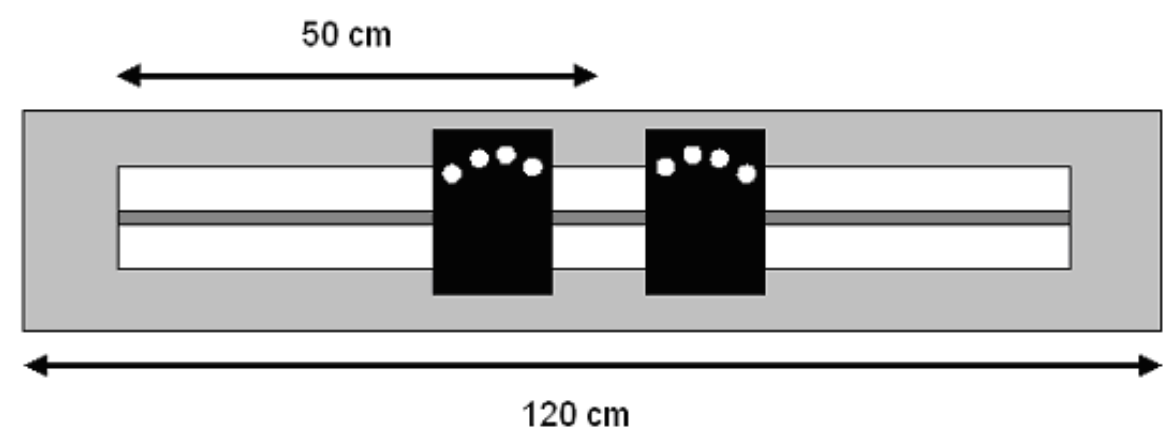

Figure 5.1. Picture of the response apparatus. Participants rested their hands on the two keypads depicted as black rectangles $(12 \times 18 \mathrm{~cm})$. In the dynamic hand condition, they continuously moved their hands back and forth at a comfortable pace. In the static hands condition, they did not move their hands. Participants had to respond as quickly as possible to the target stimulus by pressing one of the index or middle fingers of the two hands.

Participants rested their hands on moveable keypads mounted on a bar. In the static condition participants simply rested their hands on the stationary keypads, but in the dynamic condition participants continuously moved their hands back and forth while performing the finger-cuing task. The logic of this manipulation was that moving the hands would increase the level of activation in both hemispheres relative to the static condition, where the hands remain motionless. If the advantage of hand-cues relative to finger- and neither-cues is partly determined by a strong inhibition process targeted at one hemisphere, then the hand-advantage should be reduced or eliminated if this inhibition process, at least to some extent, is counteracted by an increased level of hemispheric activation generated by moving the hands.

It is relevant to note that previous work has supported the assumption that "moving the hands" increases the activation level of the hemispheres. Miller and Adam (2006) reported that redundancy gain (the phenomenon that detection responses are faster to redundant stimuli than to single stimuli) was substantially smaller when the hands were moving than when they were stationary. This finding was accounted for by two claims: (1) redundancy gain arises because of the extra response-related activation supplied to both hemispheres by redundant stimuli; and (2) the advantage due to this extra activation can be alleviated by providing an external source of activation, that is, by moving the hands. 
It is also relevant to note that the response-inhibition account of the handadvantage rests on the assumption that it is a true hand-advantage, meaning that it requires the anatomical-based hand distinction that allows for unilateral hemispheric control. Reeve and Proctor (1984, 1990; Proctor \& Reeve, 1986, 1988), however, have questioned the validity of this assumption, arguing that the hand-advantage really is an advantage for the two left-most and two right-most stimulus-response locations, not for the left or right hand per se. We defer discussion of this issue to the discussion, where we consider and reject this and other alternative explanations of the hand-advantage.

An additional issue for this study was that of practice. Participants participated in six sessions in order to examine the effect of practice on the hand motion manipulation. The rationale for this was that performing the finger-cuing task while concurrently moving the hands can be considered a dual-task situation, which may be expected to cause RT interference (e.g., Michaels \& Bongers, 1994). Training, however, can substantially reduce dual-task decrements (e.g., Ruthruff, Johnston, \& Van Selst, 2001). Hence, a second goal of this study was to examine the extent to which practice can moderate possible RT deficits in the dynamic relative to the static hands condition.

\section{METHOD}

\section{Participants}

Twelve right-handed students from Maastricht University (7 women and 5 men), with a mean age of 22.9 years (range: 19-25) participated. They were paid a small amount of money, had normal or corrected-to-normal vision, and were naïve as to the purpose of the experiment.

\section{Stimuli and Apparatus}

Participants were seated on a height-adjustable chair in front of a 13-in video monitor that was controlled by an IBM-PC compatible computer. Viewing distance was approximately $60 \mathrm{~cm}$. Participants rested their hands on two moveable keypads mounted on a bar that was placed in front of them. Each keypad was equipped with four response buttons arranged to fit the fingers of a hand (see Figure 5.1). The hands were comfortably strapped to the keypads with Velcro bands. Responses were solicited from the two index and middle fingers of each hand. Note that only these four fingers rested on the 
response buttons; the other fingers were positioned under or next to their response buttons.

Stimuli were plus signs (+). Each plus sign was approximately $2.6 \mathrm{~mm}$ wide and $3.0 \mathrm{~mm}$ high. The stimulus display consisted of a reference signal, a cue signal, and a target signal, with the entire display centered on the video monitor. The reference signal was a row of four plus signs, which was continuously present. One blank space covering $3 \mathrm{~mm}$ separated all plus signs.

\section{Procedure}

A trial started with the onset of a cue signal, which appeared immediately below the warning signal. The cue signal consisted of plus signs either in all four positions indicated by the reference signal (i.e., the uncued condition) or in only two of the four possible positions (i.e., the hand-cued, finger-cued, and neither-cued conditions). In the hand-cued condition, the cue specified two fingers on the same hand (e.g., the left-index finger and the left-middle finger). In the finger-cued condition, the cue specified the same fingers on different hands (e.g., the two index fingers). In the neither-cued condition, the cue specified different fingers on different hands (e.g., the left-middle and right-index fingers). After a constant preparation interval of $250 \mathrm{~ms}$, the target signal (a single plus sign) appeared immediately below the cue row, always in one of the positions indicated by the cue. Target signal and response key were mapped onto each other in a spatially compatible manner, such that a target appearing in the left-most position was to be responded to with the left middle finger pressing the left-most response key, etc. In the dynamic condition, participants continuously moved their hands inwards and outwards at a comfortable frequency in a self-paced manner, roughly at a frequency of about $0.67 \mathrm{~Hz}$ and with a travel distance of about $30-36 \mathrm{~cm}$ in each direction. Note that the two keypads were constrained such that they only allowed in-phase movements of the hands (i.e., the two hands always moved in opposite directions). In the static condition, the two keypads were immobilized (i.e., fixed to the bar).

In the dynamic "moving hands" condition, the presentation of the cue signal was synchronized with the position of the hands. That is, the cue signal appeared only during outward movements of the hands and when the keypads were separated by $5 \mathrm{~cm}$ or by $15 \mathrm{~cm}$ (side-to-side). Similarly, in the static hands condition, the two keypads were immobilized (i.e., fixed to the bar) with an inter-distance of either $5 \mathrm{~cm}$ or $15 \mathrm{~cm} .^{2}$ 
Participants participated in six sessions, each lasting about 35 minutes, on separate days. In each session, participants received a series of 160 trials, in each of the two hand motion conditions, with a short break halfway. Within a block of 160 trials there were 40 trials for each of the four cue conditions (uncued, hand-cued, finger-cued, and neither-cued). Within each cue condition there were 10 trials for each of the 4 stimulus positions. The order of these cue and stimulus conditions within a block of trials was random. Order of motion condition was counterbalanced.

Participants were informed regarding the nature of the task and were explicitly told to take advantage of the information provided by the cue. They were instructed to react as quickly as possible to the target stimulus by pressing the correct response key. Error feedback was provided on individual trials. At the end of each session, feedback was provided to each participant in terms of his/her overall mean RT and proportion of errors. A financial award was available for the participant with the best overall RT performance across sessions. The response pads were interfaced with the computer that recorded response latencies of each finger press response to the nearest millisecond and the position of the hands to the nearest $0.1 \mathrm{~mm}$.

\section{Analysis}

RTs below $125 \mathrm{~ms}$ or in excess of 1,000 ms were considered outliers and were excluded from all data analyzes. Using these criteria, we removed $0.31 \%$ of the trials. Mean correct RTs and proportions of errors were calculated for each subject as a function of session (1-6), motion condition (static or dynamic), and type of cue (uncued, hand-cued, finger-cued, neither-cued). An analysis of variance (ANOVA) was performed on mean RTs and percentage errors with Session, Motion Condition, and Cue Type as within-subject variables. Posthoc analyses were carried out using Tukey's least significant difference (LSD) procedure. The significance criterion was set to alpha $=.05$.

\section{RESULTS}

\section{Behavioral data.}

\section{Reaction Times}

There was a significant main effect of session, $F(5,55)=28.99, p<.001$, reflecting a substantial overall RT improvement due to practice (see Figure 5.2). This general improvement amounted to $57 \mathrm{~ms}$ or $14 \%$ from session 1 to 6 . 
The post-hoc analysis revealed that RT improved significantly during the first 4 sessions, but not thereafter. The significant Session $x$ Motion Condition interaction, $F(5,55)=3.52, p<.01$, indicated that in the first two sessions the RTs for the moving hands condition were longer than those for the static hands condition $(p<.01)$, but thereafter this difference disappeared (see Figure 5.2). Hence, early in practice a dual-task cost was evident in RT performance, but this interference effect disappeared after moderate amounts of practice in session 3.

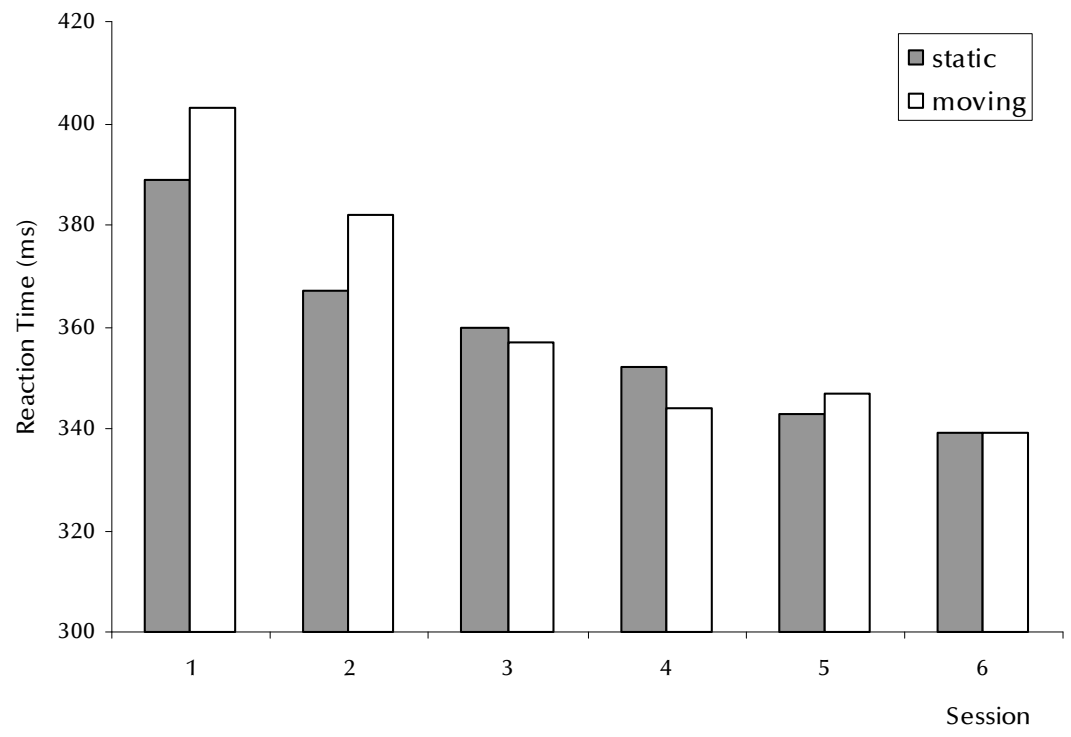

Figure 5.2. Mean reaction time in the static and moving hands conditions as a function of session.

The significant main effect of cue type, $F(3,33)=37.54, p<.001$, reflected the usual pattern of differential cuing effects, with greatest RT benefits for the hand-cued, smallest for the neither-cued, and intermediate for the fingercued condition, all showing shorter RTs compared to the uncued condition $(\mathrm{Ms}=340 \mathrm{~ms}, 365 \mathrm{~ms}, 355 \mathrm{~ms}$, and $381 \mathrm{~ms}$, respectively; all pairwise comparisons were significant, $\mathrm{p}<.05)$. Importantly, this effect was qualified by a significant Cue Type x Motion Condition interaction, $F(3,33)=5.02$, $p<.01$, which revealed an effect of motion condition that occurred only for the handcued condition (see Figure 5.3). That is, averaged over sessions, static and moving hands conditions produced similar RTs in all but the hand-cued condition, the latter showing longer RTs with the hands moving than with the 
hands static (Ms = 347 ms vs. $334 \mathrm{~ms}$, respectively; $\mathrm{p}<.001)$. In effect, whereas in the static hands condition the advantage of the hand-cued condition over the finger-cued condition was substantial $(21 \mathrm{~ms})$ and significant $(p<.001)$, in the dynamic hands condition it became smaller $(7 \mathrm{~ms})$ and non-significant $(p$ $>$.17).

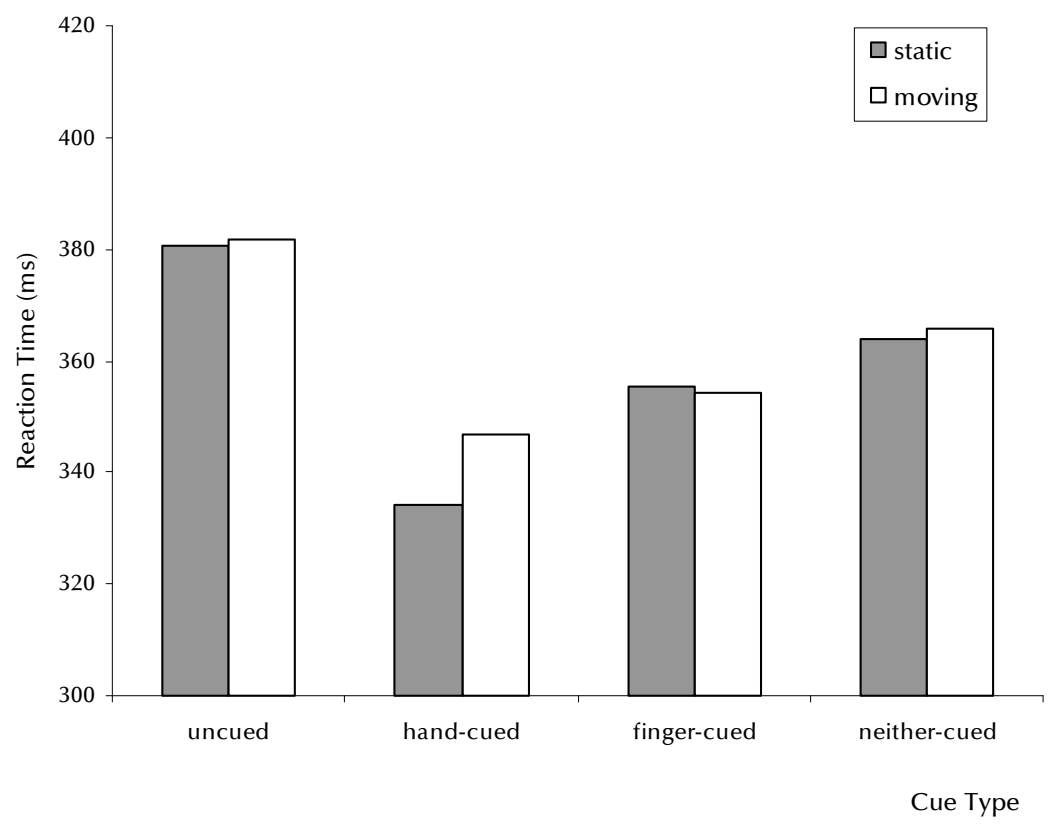

Figure 5.3. Mean reaction time as a function of cue type for the static and dynamic hand conditions.

There was also a significant Cue Type $x$ Session interaction, $F(15,165)=2.90, p$ $<.001$, which is depicted in Figure 5.4A. This interaction indicated that, averaged over motion condition, the pattern of differential cuing effects changed with practice. That is, early in practice the RT advantage of the handcued condition over both the finger- and neither-cued conditions was substantial and significant, but later in practice this hand-advantage effect was smaller and, in session 6, was eliminated relative to the finger-cued condition but not relative to the neither-cued condition. Put differently, the RT benefit (relative to the uncued condition) for the hand-cued condition remained constant across sessions, whereas it increased for the finger- and neithercued conditions, with the finger-cued condition showing a greater improvement than the neither-cued condition. This pattern of results was 
observed for both hand motion conditions, $F(15,165)<1, p>.5$, even though, overall, the cuing benefit of the hand-cued condition was smaller in the dynamic than in the static condition (see Figure 5.4B and 5.4C). Thus, with the hands moving, a significant advantage of the hand-cued condition over the finger-cued condition was observed only in the first two sessions (ps <.05), not thereafter $(p s>.2)$.
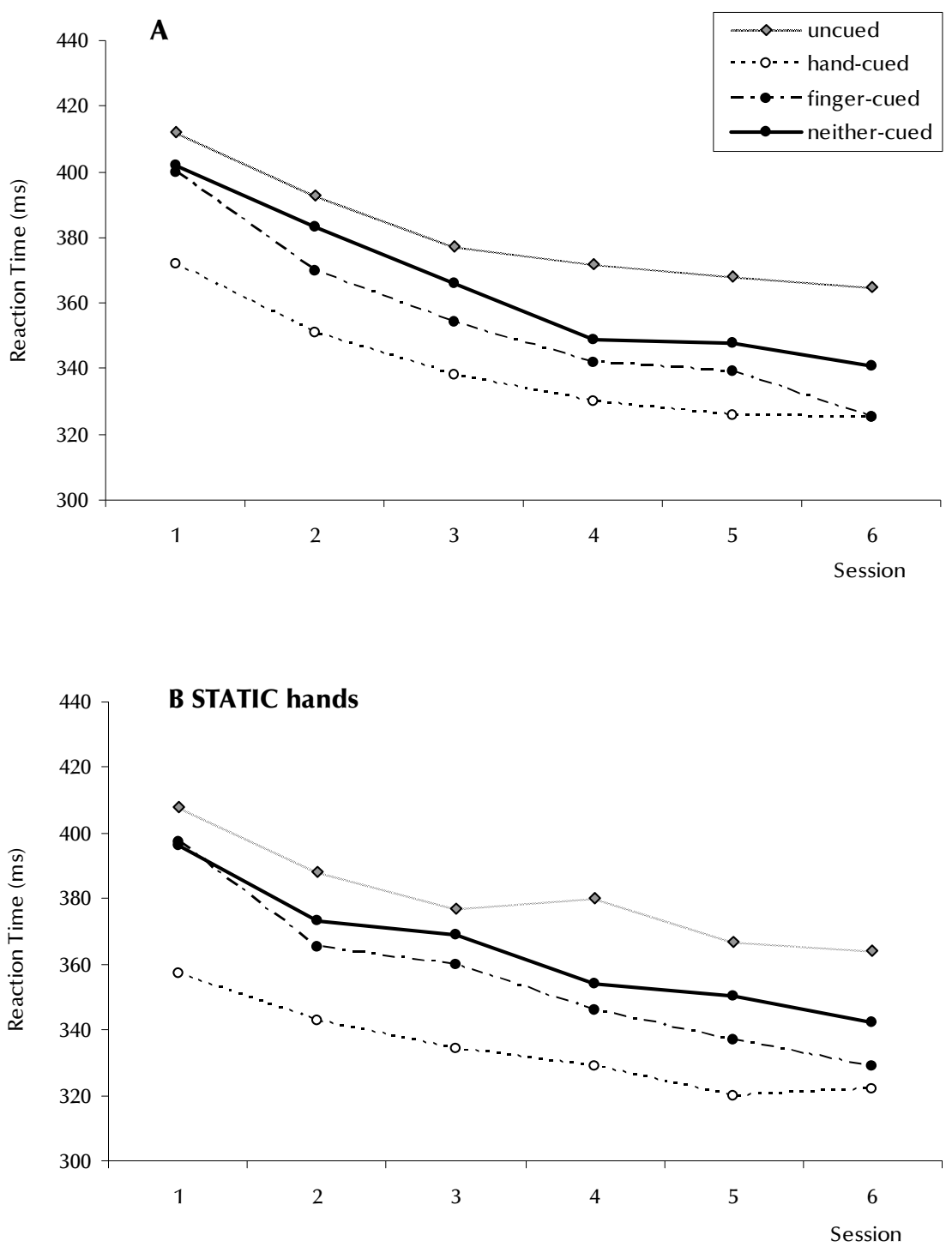


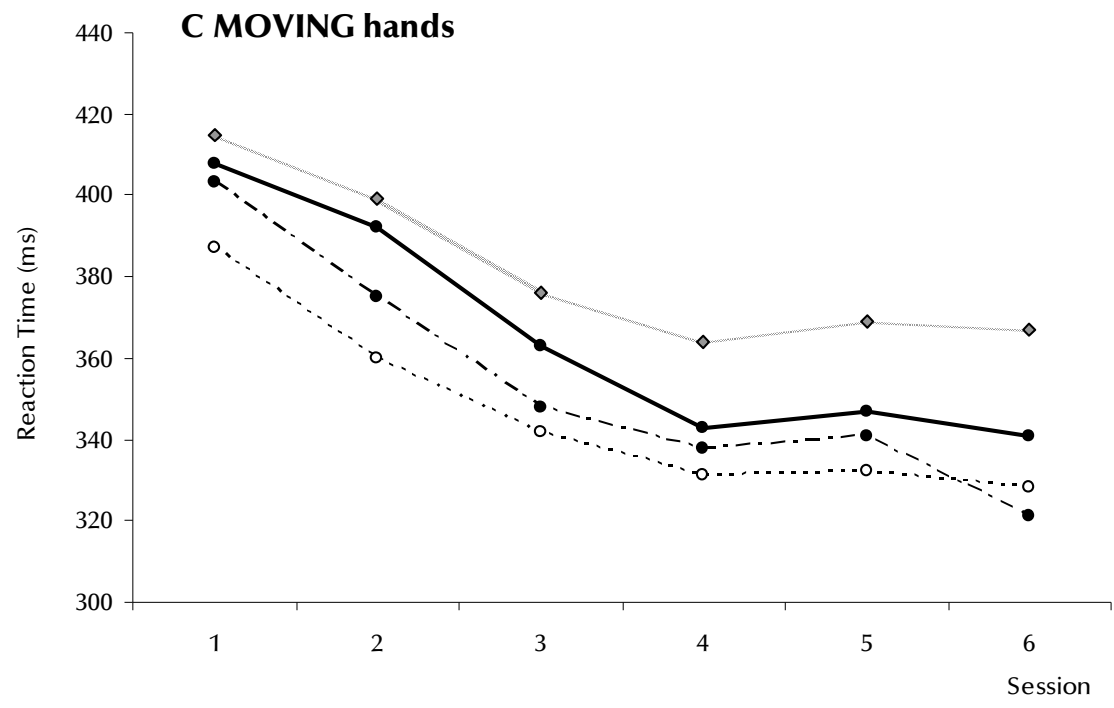

Figure 5.4. Mean reaction time as a function of cue type and session (A) averaged over the static and dynamic hand condition; (B) for the static condition; and (C) for the dynamic condition.

Errors

Mean error rate was $5.6 \%$. Error rates decreased over the first 4 sessions and stabilized thereafter: $\mathrm{Ms}=7.0 \%, 6.2 \%, 5.7 \%, 5.0 \%, 4.9 \%$, and 5.0\%, respectively; $\mathrm{F}(5,55)=3.37, \mathrm{p}<.05$. Error rates in the static and dynamic hand conditions did not differ significantly from each other: $\mathrm{Ms}=5.5 \%$ and $5.7 \%$, respectively; $F(1,11)<1, p>.6$. There were no significant interactions. 


\section{DISCUSSION}

The results were clear-cut. Moving the hands influenced only the RTs of the hand-cued condition, leaving the RTs for the finger-cued, neither-cued, and uncued conditions largely unaffected. Note, however, that the effect on the hand-cued condition was small and that the ordering of cue conditions in terms of mean RT remained the same for static and dynamic hand conditions. Nevertheless, the RT advantage for hand-cues (specifying two fingers on one hand that are represented in one hemisphere) relative to finger- and neithercues (specifying two fingers on two hands that are represented in two hemispheres) was smaller when the hands were moving than when they were stationary. As argued in the introduction, this finding of a reduced advantage for hand-cues in the dynamic condition was expected on the basis of two premises: (1) the advantage of hand-cues is due, at least in part, to a strong response inhibition process supplied to one hemisphere that effectively eliminates two of the four possible response alternatives; and (2) this efficient response inhibition process prompted by hand-cues can be counteracted by increasing the level of hemispheric activation by moving the hands. In this view, the extra activation should be specific in terms of where it accrues, namely in the neural areas concerned with the preparation and selection of irrelevant finger responses (notably the (pre)motor cortex and the supplementary motor area), and not in terms of how it accrues. As long as the neural areas involved in the selection and exaction of the irrelevant responses are supplied with extra activation, the inhibitory processes acting on these representations should be affected, and the response selection process should be compromised.

The moving hands condition required subjects to move the hands continuously back and forth in a cyclical manner, and the two response pads were mechanically constrained such that they allowed only in-phase movements of the hands. Thus, subjects performed mirror-symmetrical movements, which require simultaneous activation of the same muscle groups. It is well established that such in-phase movements are easy to control: They constitute the most basic bimanual coordination pattern, which is more accurate and stable, and requires less attention, than the anti-phase mode, which requires the alternative activation of the same muscle groups (e.g., Swinnen, 2002). Central pattern generators (i.e., relatively autonomous spinal networks) are thought to control bimanual movements, supervised and 
modulated by cortical structures, such as the supplementary motor area, the primary motor and sensory cortices, the premotor cortex and the cingulated motor area, which together constitute a distributed neural network for interlimb coordination (Swinnen, 2002). Neuroimaging studies have indicated that the easy in-phase movements show less activation in the human brain than the more difficult anti-phase movements (Swinnen, 2002). Moreover, neurological studies as well as fMRI studies have provided evidence for greater involvement of the left hemisphere in the coordination of (complex) bimanual movements (Swinnen, 2002). This notion, however, is not supported by the present study: RTs of left-hand cues and right-hand cues were very similar, if not identical (in dynamic condition: 347 and $346 \mathrm{~ms}$, respectively; in static condition: 334 and $334 \mathrm{~ms}$, respectively). Perhaps, the present in-phase bimanual coordination task was too basic to show a differential involvement of the two hemispheres.

Our finding that moving the hands affected hand-cues but not finger- and neither-cues, adds to previous evidence indicating a strong dissociation between the two types of cue: (1) hand-cues but not finger-cues are sensitive to temporal, spatial, and informative characteristics of the cue (i.e., onset vs. no-onset cues, spatial vs. symbolic cues, valid vs. invalid cues) (Adam et al., 2005); (2) finger- and neither-cues but not hand-cues show distinct developmental effects between the ages of 7 and 21 years (Adam, Parthoens, \& Pratt, 2006); and (3) hand-cues but not finger- and neither-cues are immune against the negative effects of older age (Adam, Paas, Teeken, van Loon, van Boxtel, Houx, \& Jolles, 1998; but see Proctor, Vu, \& Pick, 2006). Together, these dissociations indicate that distinct mechanisms mediate hand-cues and finger-/neither-cues.

As argued by the Grouping Model (Adam et al., 2003, 2005), hand-cues are "pull" or "exogenous" cues, prompting an automatic bottom-up process of response preparation, whereas finger- and neither-cues are "push" or "endogenous" cues, requiring an effortful top-down process to establish a selective set. The response inhibition account of the hand-advantage, which was prompted by fMRI evidence showing enhanced basal ganglia activity with hand-cues (Adam et al., 2003), suggests that the bottom-up control of handcues may, to a significant degree, be implemented via an inhibition process that suppresses the competing responses located in the opposite hemisphere. This notion is based on the proposal that the basal ganglia circuitry is a powerful output selection mechanism that acts by inhibiting irrelevant motor responses (Mink, 1996). Inhibition of the irrelevant response 
alternatives specified by finger- and neither-cues may be more difficult to achieve, because the inhibition in these cases would need to be more selective, and, moreover, distributed across the two hemispheres. The finding that the moving hands manipulation did not affect the preparation benefits generated by finger- and neither-cues fits with the suggestion of a small(er) contribution of bottom-up inhibition in these more difficult cue conditions. Further research is needed to better understand the relative contribution of activation and inhibition in response preparation as a function of cue type.

An alternative explanation for the disappearance of the hand-advantage in the dynamic condition focuses on the idea that performing the finger-cuing task while concurrently moving the hands constitutes a dual-task situation that may interfere with RT performance (e.g., Michaels \& Bongers, 1994). The results, however, indicated relatively minor dual-task interference effects that, moreover, were present only in the first two sessions and disappeared thereafter. Furthermore, the dual-task interference account has difficulty explaining the fact that the dynamic condition affected RTs only in the handcued condition, leaving RTs in the other cue conditions unaffected.

Another key outcome of this study was that practice changed the pattern of differential cuing benefits. Whereas early in practice the usual order of cuing efficiency was observed (hand-cues most effective, neither-cues least effective, and finger-cues intermediate effective), at the end of practice finger-cues were as effective as hand-cues. This outcome is consistent with research conducted by Proctor and Reeve (1988) who reported that practice can transform an initial pattern of differential cuing benefits into a pattern of equivalent cuing benefits. According to the Grouping Model (Adam et al., 2003,2005 ), these practice effects can be understood in terms of an increased efficiency in defining and matching perceptual and motoric subgroups.

It is relevant to discuss two alternative explanations of the hand-advantage. According to the "spatial proximity" hypothesis, preparation for two stimulus positions is more efficient the closer together they are, possibly because of an advantage in sharing attention across nearby positions (Miller, 1982).This hypothesis, however, can be dismissed because the observed effects do not support it. The spatial proximity hypothesis would predict shorter RTs when the index fingers are cued than when the middle fingers are cued, simply because the cue locations are in closer proximity in the former situation than in the latter. The data, however, contradict this prediction, because they show 
an opposite pattern: RTs were shorter, not longer, for preparing two middle as compared to two index fingers ( $\mathrm{Ms}=345 \mathrm{~ms}$ vs. $365 \mathrm{~ms}$, respectively).

A second alternative explanation of the advantage for hand-cues focuses on the left-right distinction in the stimulus-response displays as being critical, with the hand-distinction being of little importance (e.g., Reeve \& Proctor, 1984, 1990). From this perspective, the "hand"-advantage is due to the saliency of left-right spatial locations in the stimulus and response displays, with the specific effectors assigned to these locations being of little relevance. Using an overlapped placement of hands procedure (i.e., fingers of both hands alternating on response keys in the order: right index, left middle, right middle, left index) Reeve and Proctor (1984) reported that the usual advantage for the hand-cued condition (two fingers on one hand) turned into an advantage for the neither-cued condition (two fingers on different hands). On the basis of this finding, Reeve and Proctor (1984) argued that hand-cued advantage really is an advantage for the two leftmost and two rightmost stimulus-response locations, not for the left or right hand per se.

Adam and co-workers (Adam et al., 2003; Adam \& Van Veggel, 1992), however, noticed that Reeve and Proctor's result with the hand-placement manipulation might be restricted to, and thus an artifact of, two procedural factors: Namely, the task instructions provided to participants regarding the possibilities of preparation, and the presentation mode of the preparation intervals. In particular, Reeve and Proctor (1984) did not explicitly instruct their participants to prepare all possible finger pairings, nor did they group the different preparation intervals together in separate blocks of trials. According to Adam and Van Veggel (1992), this procedure might have favored the more natural, easy to prepare left-right cues, especially at short preparation intervals. Findings reported by Reeve and Proctor (1984, Experiment 1) supported this idea: finger- and neither-cues showed preparation benefits only with the longer preparation intervals of 1.5 and $3 \mathrm{~s}$, whereas hand-cues show preparation benefits at all preparation intervals, including the shorter preparation intervals of 375 and $750 \mathrm{~ms}$. Importantly, when Adam and Van Veggel (1992) manipulated the above two procedural factors they found that when participants are explicitly instructed to prepare all possible finger pairings, and when they know in advance how long the preparation interval is (because of a blocked presentation mode), the relative advantage of the left-right cues over the inner-outer cues is greatly reduced (by about $60 \%$ ). This is so because under these task constraints the innerouter cues produce significant RT benefits also with the shorter preparation 
intervals. Hence, Adam and Van Veggel (1992) concluded that response preparation has an important strategic component: Participants preferably engage in preparation activities when procedural constraints make it apparent, convenient and/or important to do so (see also, Requin, 1980).

Furthermore, when Adam et al. (2003, experiment 5) investigated the handplacement manipulation (adjacent versus overlapped) with the more "optimal" preparation procedures (i.e., explicitly telling the participants to prepare all possible finger pairings, and grouping the different preparation intervals together in separate blocks of trials), the advantage of the left-right cues disappeared. Thus, when the perceptually salient left-right cues are not combined with the anatomically based hand distinction, Reeve and Proctor's "left-right" advantage disappears. This point is further strengthened by evidence showing that the advantage for left-right cues disappears when the response set contains four fingers of one hand instead of two fingers of two hands (Adam et al., 2003, Experiment 1; Proctor \& Reeve, 1986, Experiment 2).Thus, the anatomically based hand distinction appears to be a prerequisite for the left-right advantage to materialize, and, hence, in effect can be considered a true hand-advantage, at least with explicit preparation instructions and blocked preparation intervals, as was the case in the present study.

In conclusion, the present results show that moving the hands can eliminate the RT advantage for preparing two fingers on one hand as opposed to preparing two (homologous) fingers on two hands. This novel finding supports the idea that activating both hemispheres by moving the hands may counteract a response inhibition process that appears to operate more effectively on response alternatives grouped together in one hemisphere than on response alternatives distributed across two hemispheres. Furthermore, the present research confirms the usefulness of the moving hand manipulation as an experimental tool to manipulate neural activity in the brain, warranting further development and application to other cognitive phenomena.

\section{FOOTNOTES}

1. Because a blocked design was used and not an event-related design, it was impossible to determine whether the increased basal ganglia activation was lateralized and consistent with to-be-inhibited response alternatives located in one hemisphere.

2. Preliminary analyses indicated that the hand-distance manipulation did not affect the results and conclusions in any meaningful way. 


\section{REFERENCES}

Adam, J.J., Backes, W., Rijcken, J., Hofman, P., Kuipers, H., \& Jolles, J. (2003). Rapid visuomotor preparation in the human brain: A functional MRI study. Cognitive Brain Research, 16, 1-10.

Adam, J.J., Hommel, B., \& Umiltà, C. (2003). Preparing for perception and action (I): The role of grouping in the response-cuing paradigm. Cognitive Psychology, 46, 302-358.

Adam, J.J., Hommel, B., \& Umiltà, C. (2005). Preparing for perception and action (II): Automatic and effortful processes in response-cueing. Visual Cognition, 12, 1444-1473.

Adam, J.J., Paas, F.G., Teeken, J.C., van Loon, E.M., van Boxtel, M.P., Houx, P.J., \& Jolles, J. (1998). Effects of age on performance in a finger-precuing task. Journal of Experimental Psychology: Human Perception and Performance, 24, 870-883.

Adam, J.J., Parthoens, S., \& Pratt, J. (2006). Distinct mechanisms for preparing keypress and reaching responses: A developmental study. Human Movement Science, 25, 293-309.

Adam, J.J., \& Van Veggel, L.M.A. (1992). Manipulating procedural variables in a spatial precuing task. Acta Psychologica, 81, 97-114.

Burle, B., Vidal, F., Tandonnet, C., \& Hasbroucq, T. (2004). Physiological evidence for response inhibition in choice reaction time tasks. Brain and Cognition, 56, 153-164.

Colby, C.L., \& Goldberg, M.E. (1999). Space and attention in parietal cortex. Annual Review of Neuroscience, 22, 319-349.

Hick, W.E. (1952). On the rate of gain of information. Quarterly Journal of Experimental Psychology, 4, 11-26.

Hyman, R. (1953). Stimulus information as a determinant of reaction time. Journal of Experimental Psychology, 45, 188-196.

Jennings, J.R., \& van der Molen, M.W. (2005). Preparation for speeded action as a psychophysiological concept. Psychological Bulletin, 131, 434-459.

Kornblum, S., Hasbroucq, T., \& Osman, A. (1990). Dimensional overlap: Cognitive basis for stimulus-response compatibility - A model and taxonomy. Psychological Review, 97, 253-270.

Leonard, J.A. (1958). Partial advance information in a choice reaction task. British Journal of Psychology, 49, 89-96.

Michaels, C.F., \& Bongers, R.M. (1994). The dependence of discrete movements on rhythmic movements: Simple reaction time during oscillatory tracking. Human Movement Science, 13, 473-493.

Miller, J. (1982). Discrete versus continuous models of human information processing: In search of partial output. Journal of Experimental Psychology: Human Perception and Performance, 8, 273 296.

Miller, J., \& Adam, J.J. (2006). Redundancy gain with static versus moving hands: A test of the hemispheric coactivation model. Acta Psychologica, 122, 1-10.

Mink, J.W. (1996). The basal ganglia: focused selection and inhibition of competing motor programs. Progress in Neurobiology, 50, 381-425.

Proctor, R.W., \& Reeve, T.G. (1986). Salient-feature coding operations in spatial precuing tasks. Journal of Experimental Psychology: Human Perception and Performance, 12, $277-285$.

Proctor, R.W., \& Reeve, T.G. (1988). The acquisition of task-specific productions and modification of declarative representations in spatial-precuing tasks. Journal of Experimental Psychology: General, 117, 182-196.

Proctor, R.W., Vu, K.-P. L., \& Pick, D.F. (2006). A deficit in older adults' effortful selection of cued responses. Journal of Motor Behavior, 38, 265-284. 
Reeve, T.G., \& Proctor, R.W. (1984). On the advance preparation of discrete finger responses. Journal of Experimental Psychology: Human Perception and Performance, 10, 541-553

Reeve, T.G., \& Proctor, R.W. (1990). The salient-features coding principle for spatial- and symboliccompatibility effects. In R. W. Proctor \& T. G. Reeve (Eds.), Stimulus-response compatibility. (pp. 163-180). Amsterdam: North-Holland.

Requin, J. (1980). Toward a psychobiology of preparation for action. In G.E. Stelmach \& J. Requin (Eds.), Tutorials in motor behavior (pp. 373-398). Amsterdam: North-Holland.

Rosenbaum, D.A. (1980). Human movement initiation: Specification of arm, direction, and extent. Journal of Experimental Psychology: General, 109, 444-474.

Swinnen, S.P. (2002). Intermanual coordination: From behavioural principles to neural-network interactions. Nature Reviews Neuroscience, 3, 348-359.

Ruthruff, E., Johnston, J.C., \& Van Selst, M. (2001). Why practice reduces dual-task interference. Journal of Experimental Psychology: Human Perception and Performance, 27, 3-21.

Usher, M., \& McClelland, J. (2001). The time course of perceptual choice: The leaky, competing accumulator model. Psychological Review, 108, 550-592. 


\section{PREPARING FINGERS WITHIN AND \\ BETWEEN HANDS IN OLDER AGE \\ CHAPTER 6}

Sofie Moresi, Jos J. Adam, Pascal W.M. Van Gerven, Barbara G. Werrij, Martin Van Boxtel \& Jelle Jolles (2009).

Accepted for publication in European Journal of Cognitive Psychology. 


\section{ABSTRACT}

Previous research has demonstrated an age-related deficit in the preparation of finger responses. A key question is whether the age-related deficit reflects differences in speed of preparation or differences in the maximal preparation benefit that can be attained given sufficiently long preparation intervals. The present study examined this issue by asking a group of younger and older adults to perform the finger-cuing task with four, relatively long, preparation intervals that varied randomly across trials. Reaction time results demonstrated that older adults were deficient in preparing two fingers on two hands at the two shortest preparation intervals, but not at the two longest ones. This outcome suggests that, with randomized preparation intervals, there is an age-related deficit in the time needed to achieve the maximal level of between-hands preparation.

\section{INTRODUCTION}

Recently, two studies have reported evidence that older age is associated with a deficit in the preparation of discrete finger responses (Adam, Paas, Teeken, van Loon, van Boxtel \& Houx et al., 1998; Proctor, Vu \& Pick, 2006). The specific nature of this deficit, however, is unclear and subject to debate. On the one hand, it could reflect the ultimate preparation benefit that can be attained given sufficiently long preparation time. On the other hand, it could reflect the preparation time needed to achieve that maximal benefit. In this paper we evaluate this issue theoretically (by examining the methodology and results of the above mentioned two papers) and empirically (by reporting the results of a new experiment).

\section{Preparation of Discrete Finger Responses}

The preparation of discrete finger responses has been studied by means of the finger-cuing task developed by Miller (1982), who adapted Rosenbaum's (1980, 1983) movement-cuing paradigm. In the finger-cuing task, three horizontal rows of symbols (representing warning, cue, and target signal) appear consecutively and underneath each other in the center of a computer monitor (see Figure 1.1). At the beginning of a trial, the warning signal (a row of four plus signs) appears for $500 \mathrm{~ms}$. Then, underneath it, the cue signal appears in the form of two plus signs, indicating a subset of two possible 
finger responses. Next, after a certain preparation interval, a single plus sign appears, signaling the required response. The response is a spatiallycompatible key press response, executed by the index or middle finger of the left or right hand.

The functional significance of the cue signal is to specify a subset of two (out of four) possible stimulus-response locations, thus converting the basic fourchoice task into a two-choice task. Four cue types can be distinguished (see Figure 1.1). In the hand-cued condition, the cue specifies two fingers on the same hand (e.g., the left-middle and left-index fingers). In the finger-cued condition, the cue specifies the same finger on two hands (e.g., the left-index and right-index fingers). In the neither-cued condition, the cue specifies different fingers on two hands (e.g., the left-index and right-middle fingers). These three preparation conditions are called the "cued" or "informative" conditions. Also, an "uncued" or "uninformative" condition is included. In this condition, no advance information about the upcoming response is provided (the four plus signs are repeated), so that selective preparation of any combination of two finger responses is precluded. In other words, the uncued condition leaves the basic four-choice task unaltered, and, thus, is a control condition against which the effects of the "cued" conditions can be evaluated. Since two-choice tasks yield substantially shorter RTs than fourchoice tasks (Hick, 1952; Hyman, 1953), cue effectiveness is inferred from a significant RT advantage or benefit for the two-choice "cued" conditions (i.e., hand-, finger-, and neither-cued) relative to the four-choice "uncued" condition.

The robust finding from the finger-cuing paradigm is a pattern of differential cuing benefits: RTs are shortest for the hand-cued condition, longest for the neither-cued condition, and intermediate for the finger-cued condition, reflecting an ordering in terms of preparation difficulty (Proctor \& Reeve, 1986; Reeve \& Proctor, 1984, 1990). Importantly, however, this pattern of differential cuing benefits is only apparent at short preparation intervals (i.e., intervals less than about $1.5 \mathrm{~s}$ ), with longer preparation intervals showing a pattern of equivalent cuing benefits. Thus, responses can be selected and prepared more quickly when they are grouped together on one hand than when they are distributed over two hands, with no differences between the three cue types given sufficiently long preparation time (for reviews see Adam, Hommel \& Umiltà, 2003; Reeve \& Proctor, 1990). 
It is relevant to note that for the purposes of the present paper it is not important to make assumptions about the origin of this "hand-advantage". Admittedly, the word "hand-advantage" appears to suggest a motoric origin, as some authors indeed have suggested (e.g., Miller, 1982). Using an overlapped placements of hands, however, Reeve and Proctor (1984, 1990) argued for a non-motoric locus, maintaining that the advantage really is a "side-advantage", that is, an advantage for the two left-most and two rightmost stimulus-response locations, not for the left or right hand per se (but see Adam et al., 2003, for a different view). Again, for the present purposes, this discussion is not relevant, as we aim to determine whether the reported age-related deficit in finger preparation reflects the maximal preparation benefit that can be achieved or the processing time needed to attain that benefit, regardless of its (motoric or non-motoric) origin.

\section{The Adam et al. (1998) Study}

In two experiments, Adam et al. (1998) examined age-related cuing effects in the finger-cuing task. In the first experiment, 140 participants drawn from six different age groups $[25,35,45,55,65$, and $75( \pm 1)$ years of age $]$, performed the finger-cuing task to determine cuing benefits generated by hand-cues relative to the control, uncued condition. There were two preparation intervals: a short $(100 \mathrm{~ms})$ and long $(2,000 \mathrm{~ms})$ preparation interval, presented in separate blocks of trials. As expected, results showed that RT benefits generated by hand-cues were substantially larger with the long than with the short preparation interval $(73 \mathrm{~ms}$ vs. $35 \mathrm{~ms}$, respectively). More importantly, however, the results also demonstrated that these hand-cued benefits were at least as large in older adults as in younger adults. Hence, the results of this first experiment demonstrated that hand-cued benefits were not negatively affected by advancing age. The second experiment attempted to obtain a more complete picture of age-related cuing benefits by adding two more cue types to the design (i.e., the more difficult finger-cued and neither-cued conditions). Also, three more preparation intervals were added, so that there were five (blocked) preparation intervals $(100 \mathrm{~ms}, 500 \mathrm{~ms}, 1,000 \mathrm{~ms}, 1,500 \mathrm{~ms}$, and $2,000 \mathrm{~ms}$ ). Twenty older adults (mean age $=71.3$ years) and twenty younger adults (mean age $=24.0$ years) participated. Results replicated the key finding of the first experiment: robust RT benefits for hand-cues that were greater for the long than for the short preparation intervals, and, more importantly, that were similar for younger and older participants, both in terms of the maximal benefit obtained and in terms of the preparation time needed to achieve this maximal benefit. In sharp contrast, however, the more difficult finger- and neither-cued conditions did show strong age-related 
deficiencies in cuing benefit: whereas younger participants showed robust finger- and neither-cued RT benefits that were equal to or approached those of hand-cues (at least with preparation intervals greater than $1 \mathrm{~s}$ ), older adults were only marginally able to reduce RT with finger- and neither-cues (with the longest preparation intervals of 1.5 and 2 s). Thus, Adam et al. (1998) concluded that older adults encounter great difficulty preparing two fingers on two hands, but not on one hand. ${ }^{1}$

At face value, the Adam et al. (1998) findings provide evidence for an agerelated deficit in the time course or speed of preparing two fingers on two hands, not per se for an age-related deficit in the maximal amount of (acrosshands) preparation that can be achieved. This is because the longest preparation interval was only $2 \mathrm{~s}$, and thus it is possible that with longer preparation intervals older adults may show equally large cuing benefits for all cue types (hand-, finger-, and neither-cues). Indeed, exactly this issue was raised and investigated by Proctor et al. (2006).

\section{The Proctor et al. (2006) Study}

Proctor et al. (2006) conducted three experiments to answer two basic questions. The first question was whether, given sufficiently long preparation intervals, there is an age-related deficit in the maximal level of preparation that can be attained when preparing fingers on different hands. The second question was whether the cuing deficit for older adults observed by Adam et al. (1998) was for fingers on different hands or for different spatial cues. To answer the first question, Proctor et al. (2006) used substantially longer preparation intervals than the ones used by Adam et al. (1998). To answer the second question, Proctor et al. (2006) used an overlapped hand placement condition next to the standard adjacent hand placement condition in order to dissociate fingers from spatial response location. As outlined earlier, the focus of the present paper is on the first issue. Hence, in the presentation and discussion of the Proctor et al. (2006) study we focus on the results pertaining to preparation benefits found with longer preparation intervals (with the adjacent hand placement condition).

Proctor's et al. (2006) first experiment was a replication of Adam's et al. (1998) second experiment, in which all cue types were used with blocked preparation intervals of $100 \mathrm{~ms}, 500 \mathrm{~ms}, 1,000 \mathrm{~ms}, 1,500 \mathrm{~ms}$, and 2,000 ms. Proctor's et al. results were comparable with those of Adam et al., showing an age-related deficit for finger- and neither-cues (at all preparation intervals), but not for hand-cues. 
Proctor's et al. (2006) more critical Experiment 2 used much longer (blocked) preparation intervals, namely $2 \mathrm{~s}, 3 \mathrm{~s}, 4 \mathrm{~s}$, and $5 \mathrm{~s}$. As expected, younger adults showed a pattern of equivalent cuing benefits for the three informative cue types (hand-, finger-, and neither-cues) at all preparation intervals. Older adults, however, still showed a distinct pattern of differential cuing benefits, with hand-cues generating significantly larger cuing benefits than finger-cues, which in turn generated larger cuing benefits than neither-cues, at all preparation intervals (mean RT benefits: $129 \mathrm{~ms}, 97 \mathrm{~ms}$, and $77 \mathrm{~ms}$, respectively). Thus, the results of this experiment revealed a solid age-related deficit in the ultimate level of preparation that can be attained when preparing two fingers on two hands, even with preparation intervals substantially longer than the $2 \mathrm{~s}$ interval used by Adam et al. (1998). It is interesting to note that Proctor et al. (2006) interpreted the results of their Experiment 2 somewhat differently. Instead of emphasizing the apparent differences in the maximal cuing benefit that can be generated by the three informative cue types (even with long preparation intervals), they underscored the finding that all cue types were able to produce large RT benefits. Even though not incorrect, this interpretation fails to recognize the relevant fact that, in older age, longer preparation intervals do not eliminate the advantage for hand-cues (over finger- and neither-cues).

In Proctor's et al. (2006) Experiments 1 and 2, the cuing or preparation interval was constant within a block of trials. In their Experiment 3 Proctor et al. (2006) varied the preparation interval randomly, so that participants were uncertain about how much time would be available for response preparation. Thus, Proctor's et al. (2006) Experiment 3 used five preparation intervals (100 ms, 500 $\mathrm{ms}, 1,000 \mathrm{~ms}, 2,000 \mathrm{~ms}$, and 5,000 ms) randomly intermixed. The results were as follows. For younger adults, the advantage for hand-cues (over finger- and neither-cues) present at the short preparation intervals disappeared completely at the longer intervals of $2 \mathrm{~s}$ and $5 \mathrm{~s}$ (in fact, it turned into a small disadvantage). For older adults, however, this did not happen because they still showed a strong and significant advantage of hand-cues (over finger- and neither-cues) at the $2 \mathrm{~s}$ preparation interval (mean RT advantage $=54 \mathrm{~ms}$ ) and a non-significant but still sizable advantage at the $5 \mathrm{~s}$ interval (mean RT advantage $=28 \mathrm{~ms}$ ). The non-significance of this latter finding, however, should be viewed with caution because of the limited number of older adults participating in this experiment $(n=8)$, which may have resulted in low statistical power. 
Taken together, the results of Proctor's et al. (2006) Experiments 2 and 3 are mixed and do not seem to solve convincingly the ambiguity present in Adam's et al. (1998) report that older adults show an age-related deficit in preparing two fingers on two hands. For all that, Proctor et al. (2006) still reached the somewhat surprising conclusion that "older adults were able to achieve the full cuing benefit shown by younger adults but required longer time to achieve the maximal benefit" (p. 265, abstract). We believe that this conclusion is at best premature and perhaps even invalid, because their Experiment 3 lacked sufficient statistical power and their Experiment 2 indicated the opposite.

\section{Purpose of Present Study}

As outlined above, the results of Proctor's et al. (2006) study do not provide an unequivocal answer to the question of whether there is an age-related deficit in the maximal preparation benefit that can be attained when preparing two fingers on different hands (given sufficiently long preparation intervals). With blocked preparation intervals, the results of Proctor's et al. Experiment 2 seem to provide solid evidence in support of such an age-related deficit (even though Proctor et al. claim that their results show otherwise). With randomly intermixed preparation intervals, however, the results of Proctor's et al. Experiment 3 seem to indicate that such an age-related deficit does not exist (at least not with the longest preparation interval of $5 \mathrm{~s}$ ), but this finding is a nul-effect and thus less convincing, especially in light of the small number of participants. To clarify this latter issue, we conducted an experiment with a substantially larger number of older and younger adults (22 participants in each group) who performed the finger-cuing task with a set of long preparation intervals $(2 \mathrm{~s}, 3 \mathrm{~s}, 4 \mathrm{~s}$, and $5 \mathrm{~s})$ that were randomly intermixed.

\section{METHOD}

\section{Participants}

Two groups of adults participated: 22 younger adults, aged between 18 and 24 years (12 men, $M=22.2$ years, $S D=2.3$ ) and 22 older adults, aged between 65 and 80 years ( 9 men, $M=71.4$ years, $S D=3.5$ ). Younger and older adults were recruited via posters and via a database of volunteers available for research at the Department of Psychiatry and Neuropsychology of Maastricht University. Exclusion criteria were previous or actual medical conditions with known impact on cognitive, motor or both functions: chronic neurological pathology (history of a cerebrovascular accident, epilepsy, dementia, or Parkinson's 
disease), mental retardation, ophthalmologic diseases (e.g., retinopathy, cataract) or bone deformities of the fingers (e.g., arthrosis and rheumatoid arthritis). Moreover, participants were not allowed to use psychotropic drugs, excessive alcohol. The same exclusion criteria were applicable for the younger adults. Except for two younger men and one older woman, all subjects were right handed, as determined by self report. All participants had normal or corrected-to-normal vision. The experiment was approved by the Ethical Committee of the Faculty of Psychology. Informed consent was obtained and all participants received $€ 10$ for their participation. Data for IQ were collected by means of an abbreviated version of the standard Dutch intelligence test (Luteijn \& van der Ploeg, 1983). The two age groups were not reliably different in terms of $\mathrm{IQ}, \mathrm{t}(42)=.89, \mathrm{p}>.05[\mathrm{Ms}=116.8$ (5.6) and 119.0.6 (9.8) for younger and older adults, respectively].

\section{Stimuli and apparatus}

Participants were seated in a height adjustable chair in front of a 16-in. monitor controlled by a Compaq Presario 7170 computer. Viewing distance was approximately $50 \mathrm{~cm}$. Responses were made by pressing one of four adjacent keys in the middle of the bottom row of a standard keyboard $(\mathrm{V}, \mathrm{B}$, $\mathrm{N}, \mathrm{M})$.

Stimuli were plus (+) signs of the standard ASCII character set. Each plus sign was approximately $6 \mathrm{~mm}$ wide and $3 \mathrm{~mm}$ high $(0.69$ and 0.34 degrees of visual angle, respectively). Stimuli were presented in the middle of the display and consisted of a warning signal, a cue signal, and a target stimulus (see Figure 1.1). The warning signal consisted of four plus signs separated by $15 \mathrm{~mm}$. After a delay of $500 \mathrm{~ms}$, the cue signal appeared exactly below the warning signal. The cue consisted of plus signs in either all four positions (uncued condition) or in just two of the four positions (cued conditions). Then, after a variable preparation interval of $2 \mathrm{~s}, 3 \mathrm{~s}, 4 \mathrm{~s}$, or $5 \mathrm{~s}$, the target stimulus was presented exactly below one of the cued positions.

\section{Design and procedure}

Twenty practice trials preceded the experiment to familiarize participants with the task. Then each participant received four blocks of each 80 experimental trials, separated by a break of $1 \mathrm{~min}$, totalling 320 trials. There were 20 trials for each cue type at each preparation interval. The order of the 4 (cue type) $x 4$ (preparation intervals) $=16$ conditions over the 320 trials was random. Participants were informed regarding the nature of the task and were explicitly told to take advantage of the cue by preparing the indicated 
responses. They were instructed to react as quickly and accurately as possible by pressing the correct response key. When pressing an incorrect response key, an error message was presented on the screen. The inter-trial interval was $1 \mathrm{~s}$.

\section{Analysis}

Reaction times (RTs) less than $150 \mathrm{~ms}$ or in excess of $1250 \mathrm{~ms}$ ( $1.6 \%$ of all trials) were considered outliers and excluded from data analysis. Mean (correct) RT and percentage of errors were calculated for each participant as a function of cue type and preparation interval. A mixed-model ANOVA was performed on mean RT and on the arcsine-transformed error rates, with age (young/old) as between-groups variable, and preparation interval $(2,3,4$, and $5 \mathrm{~s})$ and cue type (uncued, hand-cued, finger-cued, and neither-cued) as within-groups variables.

\section{RESULTS}

\section{Behavioral data}

\section{Reaction Time}

All main effects were significant. The main effect of age indicated longer RTs for older than for younger adults, $F(1,42)=107.01, p<.001 ; M=661 \mathrm{~ms}$ and $405 \mathrm{~ms}$, respectively. The main effect of preparation interval indicated shorter RTs for longer preparation intervals, $F(3,126)=107.01 \mathrm{p}<.001 ; \mathrm{M}=574 \mathrm{~ms}, 530$ $\mathrm{ms}, 516 \mathrm{~ms}$, and $512 \mathrm{~ms}$ for the 2, 3, 4, and $5 \mathrm{~s}$ preparation interval, respectively. The main effect of cue type reflected shorter RTs for all informative cues relative to the uninformative cue, $F(3,126)=85.38, p<.001$; $M=507 \mathrm{~ms}, 515 \mathrm{~ms}, 522 \mathrm{~ms}$, and $588 \mathrm{~ms}$ for hand-cued, finger-cued, neithercued, and uncued, respectively. These main effects were qualified by two interactions. 


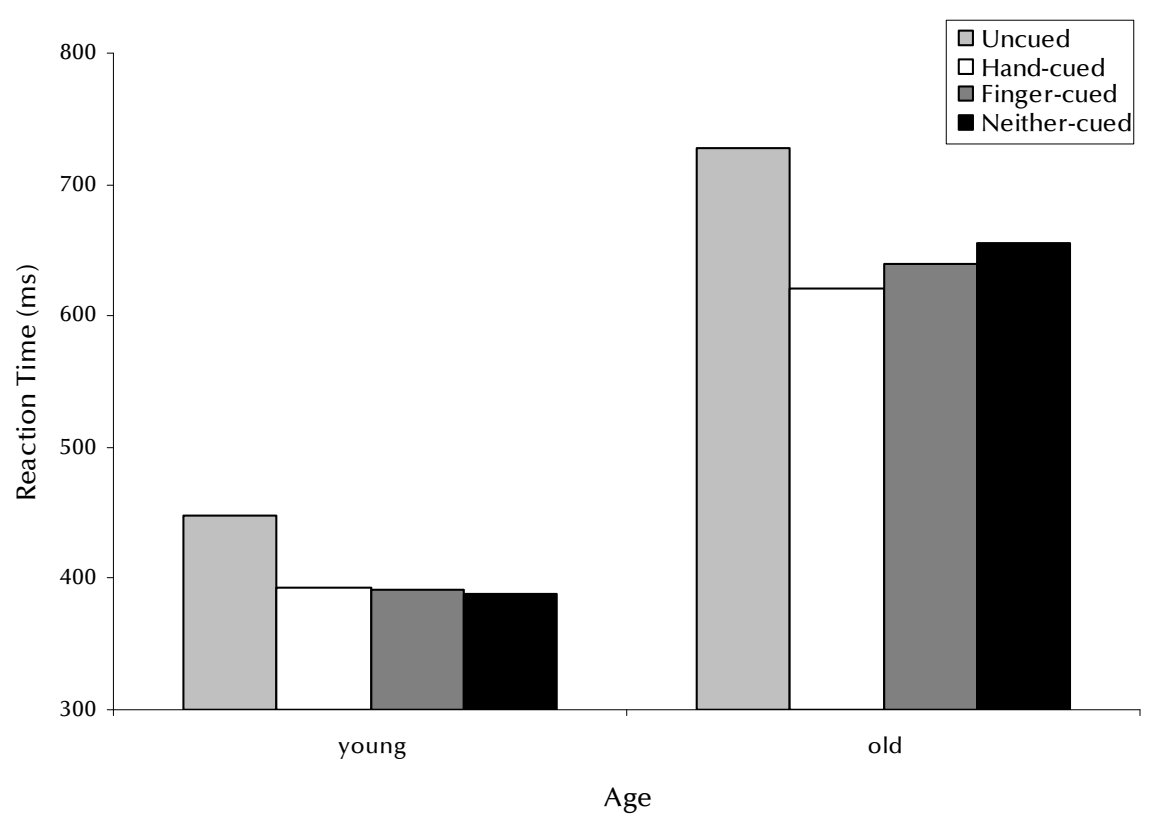

Figure 6.1. Mean reaction time (ms) as a function of cue type for younger and older participants.

The Age $x$ Cue Type interaction, $F(3,126)=8.45, \mathrm{p}<.001$, depicted in Figure 6.1, reflected in part an overall larger cuing benefit for older than younger adults $(90 \mathrm{~ms}$ vs. $57 \mathrm{~ms}, \mathrm{p}<.01)$. Furthermore, it indicated that younger adults showed a pattern of equivalent RT benefits (i.e., similar RTs for hand-, finger-, and neither-cued conditions: $M=394 \mathrm{~ms}, 391 \mathrm{~ms}$, and $388 \mathrm{~ms}$ respectively; $\mathrm{p}$ > .5), whereas older adults showed a pattern of differential RT benefits (i.e., a statistically robust RT advantage for hand-cues over both finger- and neithercues: $M=621 \mathrm{~ms}, 639 \mathrm{~ms}$, and $656 \mathrm{~ms}$, respectively; $\mathrm{p}<.001)$. Importantly, the significant Age $x$ Cue Type $x$ Preparation Interval interaction, $F(9,378)=2.30$, $p$ $<.05$, qualified this picture further by indicating that the pattern of equivalent RT benefits for younger adults was independent of preparation interval $(p>$ .3), whereas the RT advantage for hand-cues for older adults emerged at the two shortest preparation intervals ( $p s<.05$ ), but not at the two longest preparation intervals ( $\mathrm{ps}>$.1) (see Figure 6.2). These findings indicate that with randomly intermixed preparation intervals older adults can prepare two fingers on two hands equally well as two fingers on one hand, but that they require more time to do so. 


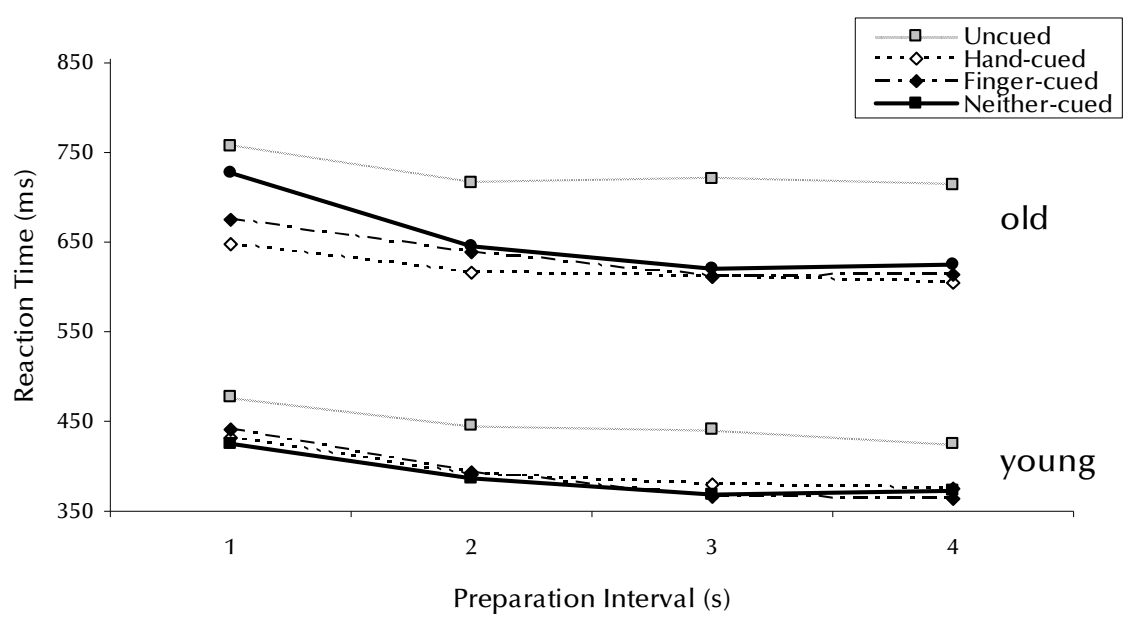

Figure 6.2. Mean reaction time (ms) as a function of cue type and preparation interval for younger and older participants.

Errors

Overall mean error rate was: $2.0 \%$. The ANOVA demonstrated significant main effects of age, $F(1,42)=15.75 p<.001$, cue type, $F(3,126)=4.06, p<.05$, and preparation interval, $F(3,126)=3.59 \mathrm{p}<.05$. These main effects indicated that younger adults made more errors than older adults $(M=2.9 \%$ vs. $1.1 \%$, respectively), that the uncued condition was more error-prone than the hand, finger-, and neither-cued conditions $(\mathrm{M}=2.8 \%, 1.5 \%, 1.8 \%$, and $1.9 \%$, respectively), and that fewer errors occurred in the longest preparation interval of $5 \mathrm{~s}$ than in the shorter intervals $(\mathrm{M}=2.2 \%, 2.1 \%, 2.3 \%$, and $1.3 \%$, for increasing intervals, respectively). These main effects, however, were qualified by two interactions.

The Age x Preparation Interval interaction, $F(3,126)=2.87, p<.05$, indicated that only younger adults showed an effect of preparation interval $(\mathrm{M}=3.5 \%$, $3.0 \%, 3.5 \%$, and $1.7 \%$ for increasing intervals, respectively; $p<.01$ ), older adults not $(\mathrm{M}=1.0 \%, 1.2 \%, 1.1 \%$, and $1.0 \%$ for increasing intervals, respectively; $p>$.9). The Age $x$ Cue Type interaction, $F(3,126)=8.28, p<.001$, depicted in Figure 6.3, indicated that younger adults made more errors in the uncued condition than in the three informative cue conditions $(p<.01)$, whereas older adults made fewer errors in the uncued condition than in the finger- and neither-cued conditions $(p<.01)$. This finding may suggest that the overall greater cuing benefit (in terms of RT) for older than for younger 
adults can be explained in terms of a speed-accuracy tradeoff. In this interpretation, older adults made least errors in the uncued condition, and this bias for accuracy in this condition may have lengthened the "uncued" RTs, thereby inflating the cuing benefit in older adults. Younger adults, on the other hand, produced most errors in the uncued condition, and this bias for speed in this condition, may have shortened the "uncued" RTs, thereby deflating the cuing benefit in younger adults.

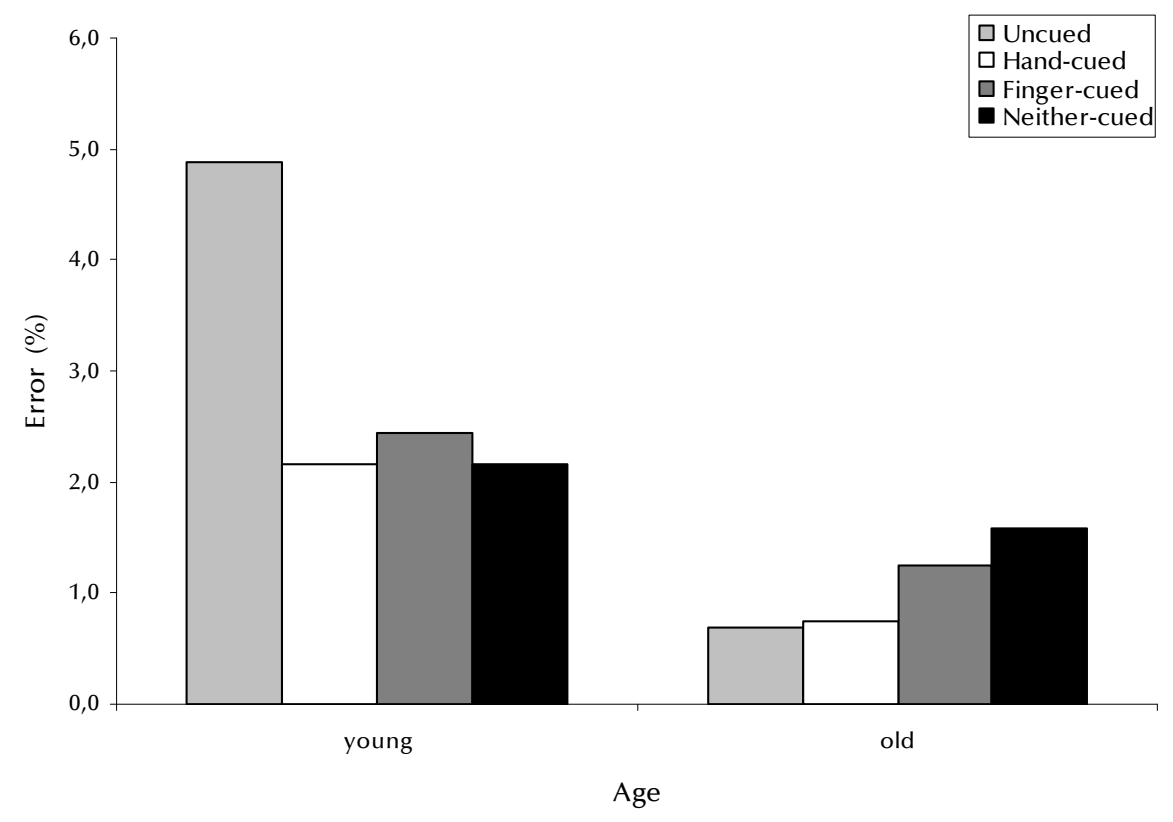

Figure 6.3. Mean error rate (\%) as a function of cue type for younger and older participants.

\section{DISCUSSION}

Following the report by Adam et al. (1998) that older adults experience difficulty in preparing two fingers on two hands, but not on one hand, it was unclear whether this age-related deficit concerned the maximal preparation benefit that can be attained or the time needed to achieve that maximal benefit. Investigating this issue, Proctor et al. (2006, Experiment 2) used much longer preparation intervals than the ones used by Adam et al. (1998), but still found, for older adults, a greater preparation benefit for cues specifying fingers on one hand than for cues specifying fingers on two hands. Thus, in effect Proctor et al. (2006, Experiment 2) demonstrated an age-related deficit 
in the maximal level of preparation that can be attained when preparing two fingers on two hands. As noted before, however, Proctor et al. (2006) insufficiently appreciated this outcome. Instead, they emphasized the fact that all informative cues generated large cuing benefits relative to the uncued condition, concluding, in our view not too convincingly, that there was little evidence of an age-related deficit in preparing responses on two hands as opposed to one, at least in terms of the ultimate level of preparation that can be achieved (for a similar conclusion, see Proctor, Vu, \& Pick, 2005). We believe, however, that the persistent hand-advantage with longer preparation intervals in older age demonstrated by Proctor et al. (2006, Experiment 2) should not be overlooked but instead should be brought into sharp focus because it has important theoretical and practical implications. That is, theories about response preparation and aging should be able to explain the finding that, under conditions of sufficient and predictable preparation time, the hand-advantage does not disappear in older age. For one thing, this finding appears to suggest that aging brings about an intrinsic limit in between-hands preparation relative to within-hands preparation.

According to the Grouping Model of finger-cuing effects (Adam et al., 2003, Adam Hommel \& Umiltà, 2005), which is an extension of the salient-features coding principle (Reeve \& Proctor, 1990), the hand-advantage reflects the strong, natural, bottom-up grouping of the hand or left-right cues, which allow fast, automatic selection of left-side or right-side responses. In contrast, bilateral finger- and neither-cues contain stimulus-response elements on both sides of the perceptual-motor work space that are not easily coded as belonging to a group. This is because these elements intrinsically belong to different groups (i.e., the left-right groups). Hence, top-down controlled processing is needed to break or overrule the low-level, bottom-up formation of left-right perceptual-motor subgroups. Thus, when grouping is weak, ambiguous or complex - as is the case with the finger- and neither-cues-it must be supported by a slow, effortful top-down process. Assuming that advancing age is accompanied by a reduction in central resources while leaving automatic processes intact (e.g., Allen, Groth, Weber \& Madden, 1993; Ford, Roth, Isaacks, Tinklenberg, Yesavage \& Pfefferbaum, 1997; Hasher \& Zacks, 1979; Wishart, Lee, Murdoch \& Hodges, 2000) the persistent handadvantage in older age demonstrated by Proctor et al. (2006, Experiment 2) accords with the notion that hand-cues prompt the fast, bottom-up selection and preparation of fingers, whereas the more difficult finger- and neithercues require slow and effortful top-down processes to establish a selective preparatory set. 
As noted in the introduction, in Proctor's et al. (2006) Experiment 2, which used blocked preparation intervals, the "hand-advantage" in older age was robust and significant, but it was smaller and non-significant in their Experiment 3, which used random preparation intervals. A possible limitation of this last experiment, however, was the small number of participants $(n=8)$. To overcome this limitation the present experiment used a much larger sample size (22 participants in each age group), in combination with randomly intermixed (long: 2-5 s) preparation intervals. Our RT results demonstrated, for older adults, that the hand-advantage, present with the two shortest preparation intervals of $2 \mathrm{~s}$ and $3 \mathrm{~s}$, disappeared with the two longest preparation intervals of $4 \mathrm{~s}$ and $5 \mathrm{~s}$. Thus, with randomized preparation intervals, older adults can prepare two fingers on two hands to the same extent as two fingers on two hands, but they require more time to do so.

When combined, the results of Proctor et al. (2006) and the present findings reveal an interesting paradox. With blocked (Proctor et al., 2006, Experiment 2) but not with randomized (present study; Proctor et al., 2006, Experiment 3) preparation intervals of long duration older adults show a deficit in the maximal level of preparation benefit that can be attained when preparing two fingers on two hands as opposed to preparing two fingers on one hand. This divergent set of findings adds to a large body of research demonstrating that the way preparation intervals are presented - i.e., mixed or not mixed in experimental blocks of trials - can have a strong influence on task performance (see Los, 1996, for a review).

With blocked preparation intervals, participants know in advance how much time there is available to select and prepare the cued finger responses before the target appears. In contrast, with randomized preparation intervals, participants do not know how much time is available for response preparation. In other words, with randomized preparation intervals there is more time uncertainty, which makes preparation more difficult, and thus could introduce differences in preparation strategy and efficiency. Indeed, with blocked preparation intervals, RT for all cue types tended to increase as a function of longer preparation intervals (Adam et al., 1998; Proctor et al., 2006, Experiment 1 and 2), whereas with randomized preparation intervals RT tended to decrease as a function of longer preparation intervals (present study; Proctor et al., 2006, Experiment 3). This pattern is robust, as demonstrated by many studies (see Niemi \& Näätänen, 1981, for a review).The first phenomenon has been interpreted as reflecting a difficulty in 
maintaining a general readiness, or set, to respond (e.g., Gottsdanker, 1975). The second phenomenon has been interpreted as indicating that participants increase their level of preparation with increasing preparation interval, because as the preparation interval lengthens the probability that the target will appear increases too (e.g., Stilitz, 1972).

On this conception, relatively long and fixed preparation intervals may allow participants to develop and implement optimal preparation strategies that ensure maximal RT benefits at all preparation intervals. Hence, the fact that older adults show a greater preparation benefit for hand-cues than for fingerand neither-cues, even with sufficient time to prepare (Proctor et al., 2006, Experiment 2), is an important finding, because it suggests that there is an intrinsic, structural limit in between-hands preparation compared to withinhand preparation as a result of aging. As noted before, this limit may be related to a reduction in central resources (e.g., Hasher \& Zacks, 1979).

When the duration of the preparation interval varies unpredictably from trial to trial, participants may adopt a strategy of increasing their level of preparation with longer preparation intervals because they know that the probability that the target will appear increases. In this view, preparation is suboptimal at short (randomized) preparation intervals and improves with longer preparation intervals. This view is consistent with a recent study by Bherer and Belleville (2004), who showed that older people more than younger people tend to direct their preparatory activities toward the moments of high probability, and with mixed preparation intervals those moments correspond to the longer preparation intervals. This phenomenon fits with the present finding that the overall preparation benefit for older adults increased with longer randomized preparation intervals (but not with longer blocked preparation intervals in Proctor's et al. Experiment 2). Moreover, this increase in preparation efficiency with longer preparation intervals was most prominent for the difficult finger-and neither-cues, and minor or absent for the easy hand-cues (see Figure 3). This outcome accords with the Grouping Model's processing assumption that the preparation of fingers on different hands depends on the investment of effortful, top-down processes, whereas the preparation of fingers on the same hand proceeds in a more bottom-up, automatic manner (Adam et al., 2003, 2005).

In conclusion, the present paper contributes in two important ways to the literature on aging and response preparation. First, it provides a theoretical clarification of Proctor's et al. (2006) Experiment 2 by noting that although 
longer preparation intervals did reduce the preparation advantage for handcues compared to finger- and neither-cues, they did not fully eliminate it, suggesting that effortful, top-down preparatory processes may be structurally affected and restricted in older age. Second, it provides an empirical clarification of Proctor's et al. (2006) Experiment 3 by providing more solid evidence that the use of randomized preparation intervals abolishes the hand-advantage in older age. Further research, preferably using a sensitive within-subject design, will be necessary to clarify the complex relationship between presentation mode of preparation interval (blocked vs. random) and type of preparation (within- vs. across-hands) in achieving the maximal preparation benefit in older age.

\section{FOOTNOTES}

1. Again, it should be noted that this conclusion should not be taken literally as it does not necessarily imply an anatomical (i.e., hand) basis for the deficit. Indeed, Adam et al. (1998) explicitly acknowledged the work of Reeve and Proctor (1984, Experiment 3), who, using an overlapped placement of hands (i.e., fingers of both hands alternating on response keys in the following order: right index, left middle, right middle, left index), reported evidence that the usual advantage of hand-cues (two fingers on one hand) switches to an advantage for neither-cues (different fingers on different hands). This outcome suggests that the handcued advantage is in fact an advantage for the two left-most and two right-most stimulusresponse locations, not for the left or right hand per se. Adam et al. (2003), however, argued that this left-right advantage is restricted to, and thus an artifact of, two procedural factors, namely instructions to participants (explicit vs. implicit preparation instructions) and presentation mode of preparation intervals (random vs. blocked). For the present purposes, however, it is important to reiterate that it is immaterial whether the hand-advantage and the age-related deficit have an anatomical or spatial basis.

\section{REFERENCES}

Adam, J. J., Hommel, B., \& Umiltà, C. (2003). Preparing for perception and action (I): the role of grouping in the response-cuing paradigm. Cognitive Psychology, 46, 302-358.

Adam, J. J., Hommel, B., \& Umiltà, C. (2005). Preparing for perception and action (II) Automatic and effortful Processes in Response cuing. Visual Cognition, 12, 1444-1473.

Adam, J. J., Paas, F. G., Teeken, J. C., van Loon, E. M., van Boxtel, M. P., Houx, P. J., et al. (1998). Effects of age on performance in a finger-precuing task. Journal of Experimental Psychology: Human perception and performance, 24, 870-883.

Allen, P. A., Madden, D. J., Weber, T. A., \& Groth, K. E. (1993). Influence of age and processing stage on visual word recognition. Psychological Aging, 8, 274-282.

Bherer, L., \& Belleville, S. (2004). Age-related differences in response preparation: The role of time uncertainty. Journal of Gerontology Series B: Psychological Sciences and Social Sciences, 59, P66-P74. 
Ford, J. M., Roth, W. T., Isaacks, B. G., Tinklenberg, J. R., Yesavage, J., \& Pfefferbaum, A. (1997). Automatic and effortful processing in aging and dementia: event-related brain potentials. Neurobiological Aging, 18, 169-180.

Gottsdanker, R. (1975). The attaining and maintaining of preparation. In P. M. A. R. S. Dornic (Ed.), Attention \& Performance V (pp. 33-49). London: Academic Press.

Hasher, L., \& Zacks, R. T. (1979). Automatic and effortful processes in memory. Journal of Experimental Psychology: General, 108, 356-388.

Hick, W. E. (1952). On the rate of gain of information. Quarterly Journal of Experimental Psychology, 4, 11-26.

Hyman, R. (1953). Stimulus information as a determinant of reaction time. Journal of Experimental Psychology, 45, 188-196.

Los, S. A. (1996). On the origin of mixing costs: Exploring information processing in pure and mixed blocks of trials. Acta Psychologica, 94,145-188.

Luteijn, F., \& van der Ploeg, F. A. E. (1983). Handleiding Groniger Intelligentie Test, GIT [Manual of the Groniger Intelligence Test] Lisse, The Netherlands: Swets \& Zeitlinger.

Miller, J. (1982). Discrete versus continuous stage models of human information processing: in search of partial output. Journal of Experimental Psychology: Human perception and performance, 8, 273-296.

Niemi, P., \& Näätänen, R. (1981). Foreperiod and simple reaction time. Psychological Bulletin, 89, 133-162.

Proctor, R. W., Vu, K. P., \& Pick, D. F. (2005). Aging and response selection in spatial choice tasks. Human Factors, 47, 250-270.

Proctor, R. W., Vu, K. P., \& Pick, D. F. (2006). A deficit in older adults' effortful selection of cued responses. Journal of Motor Behavior, 38, 265-284.

Reeve, T. G., \& Proctor, R. W. (1984). On the advance preparation of discrete finger responses. Journal of Experimental Psychology: Human Perception and Performance, 10, 541-553.

Reeve, T. G., \& Proctor, R. W. (1990). The salient-features coding principle for spatial-and symboliccompatibility effects. In R. W. Proctor \& T. G. Reeve (Eds.), Stimulus-response compatibility (pp. 163-180). Amsterdam: North-Holland.

Rosenbaum, D. A. (1980). Human movement initiation: specification of arm, direction, and extent. Journal of Experimental Psychology: General, 109, 444-474.

Rosenbaum, D. A. (1983). The movement precuing technique: assumptions, applications, and extensions. In R. Magill (Ed.), Memory and Control of Action (pp. 231-274): North-Holland publishing company.

Smith, G.A., \& Brewer, B. (1995). Slowness and age: Speed-accuracy mechanisms. Psychology and Aging, 10, 238-247.

Strayer, D.L., \& Kramer, A.F. (1994). Aging and skill acquisition: Learning-performance. distinctions. Psychology and Aging, 9, 589-605.

Stilitz, I. (1972). Conditional probability and components of RT in the variable foreperiod experiment. Quarterly Journal of Experimental Psychology, 24, 159-168.

Wishart, L. R., Lee, T. D., Murdoch, J. E., \& Hodges, N. J. (2000). Effects of aging on automatic and effortful processes in bimanual coordination. Journals of Gerontology: psychological sciences and social sciences, 55, P85-94. 
EPILOGUE

\section{CHAPTER 7}

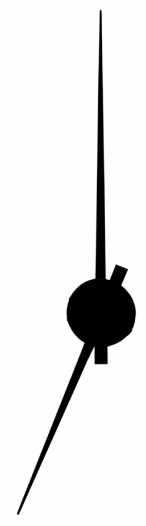




\section{EPILOGUE}

The ability to rapidly adjust perceptual-motor behavior to changing environmental demands is crucial for successful performance. Adaptive motor behavior requires brain processes that selectively make sensory and motor systems more efficient for handling an upcoming stimulus and preparing the most appropriate response. These processes are commonly referred to as response preparation. In the current thesis, we reported a series of experimental studies that sought to investigate the processes underlying response preparation in a finger-cuing paradigm by using a behavioral (RT) and a psychophysiological measure (pupil dilation).

A recent account of finger preparation is the Grouping Model (Adam et al., 2003b, 2005), which is based on two assumptions: the grouping and the processing assumption. All studies in the present thesis were aimed at testing these two assumptions in order to provide converging and independent evidence for the Grouping Model.

\section{Independent evidence for the Grouping Model}

One of the limitations of the Grouping Model is the circularity in defining a "good group" (Adam et al., 2003b). The concept of "good grouping" (i.e., relative relatedness or associative strength), as defined in the tradition of Gestalt psychology, refers to properties like proximity, symmetry, and simplicity. Unfortunately, application of these principles to assume some but not other groupings is sometimes difficult to justify in advance. This is a weak spot because the predictive power of a model depends on an a priori method of describing the strength of grouping across a wide variety of stimulus and response sets. Adam et al. (2003b) suggested two ways to overcome these limitations: (a) developing formal computational models of grouping strength that allow precise, quantitative predictions and (b) examining the "good grouping" assumption with independent tests.

In the current thesis, we chose the latter approach. We aimed to collect independent evidence for the grouping and the processing assumption. Pupillometry (Chapters 2, 3, and 4), the "moving hands" manipulation (Chapter 5), and aging (Chapter 6) were used as independent tests. The next section describes the main findings of each study according to the assumption they tested. 


\section{The grouping assumption}

According to the grouping assumption, the pattern of differential cuing benefits is mediated by interacting perceptual and motoric grouping factors in the stimulus- and response sets, respectively. In our second pupillometric study (Chapter 3; Moresi et al., submitted), we varied the response set using an overlapped hand placement next to the conventional adjacent hand placement. Results showed longer RTs and larger pupil dilations for the overlapped than for the adjacent hand placement, reflecting an overall increase in cognitive processing load. Importantly, pupil data showed that with the hands adjacent, pupil dilation was smaller for hand-cues than for neither-cues, but with the hands overlapped, this advantage for hand-cues disappeared. This outcome supports the notion that the anatomical hand distinction is an important determinant of finger-cuing efficiency. Note that this conclusion supports the Grouping Model, which highlights the role of motoric factors in response preparation, but disqualifies the salient-features coding principle (Reeve \& Proctor, 1984, 1985, 1990), which accentuates the role of spatial factors.

\section{The processing assumption}

According to the processing assumption, automatic and effortful processes mediate the pattern of differential response-cuing effects. On the one hand, fast, automatic selection of the cued responses occurs when the cue indicates a strong, good subgroup of stimuli that corresponds closely with a strong and similar grouping of responses. On the other hand, a slower, effortful process is required to create good, finely tuned subgroups when the cue indicates stimuli or responses belonging to different subgroups, or if there is a mismatch between the grouping of stimuli and the grouping of responses. Four of our studies (Chapters 2, 4, 5, and 6) supplied independent evidence for this assumption.

In our first study (Chapter 2; Moresi et al., 2008b), the difficulty of response preparation was manipulated by cuing two fingers on one hand (hand-cued), two homologous fingers on two hands (finger-cued), and two nonhomologous fingers on two hands (neither-cued). We examined whether this pattern of cue difficulty (hand-cued, finger-cued, and neither-cued) was reflected by pupil dilation as an index of processing load. Our results showed that the selection and preparation of the more difficult, bilateral response sets (finger-and neither-cued) led to larger pupil dilation than the preparation of 
the easier, unilateral response set (hand-cued), suggesting that betweenhands preparation is more effortful than within-hands preparation.

In the third study (Chapter 4; Moresi et al., 2008a), pupil dilation was used to investigate the mental effort associated with valid and invalid response preparation. Because invalid cues incorrectly specify fingers, reprogramming or restructuring operations are required to suppress wrongly prepared responses and reactivate already suppressed ones. Consistent with this premise, overall, pupil dilations were stronger for invalid than for valid cues. Furthermore, restructuring operations might be especially effortful for handcues, as shown by a functional neuroimaging study (Adam et al., 2003a). Handcues are associated with stronger basal ganglia activity than finger- and neither-cues, suggesting stronger inhibition of irrelevant responses grouped together in one hemisphere. Indeed, our pupil data showed that invalid handcues were associated with greater pupil dilations than invalid finger- and neither-cues, suggesting that the correction of automatic, bottom-up processes requires more effort than the correction of slow, central top-down processes.

In the fourth study (Chapter 5; Adam \& Moresi, 2007), we further investigated the role of inhibition in finger preparation using a moving-hands response set next to the standard static-hands response set. As described in the previous paragraph, Adam et al. (2003a) suggested that the mechanism of responseinhibition might play a key role in producing the hand-advantage. According to this account, the advantage of preparing two fingers on one hand (represented in one hemisphere) as opposed to preparing two fingers on two hands (represented in two hemispheres) is partly due to a response inhibition process that operates more efficiently within than between hemispheres. During between-hemisphere preparation (finger- and neither-cues), relevant and irrelevant responses are represented by directly adjacent and overlapping cortical finger representations (e.g., Dechent \& Frahm, 2003). This necessitates a finely tuned spatial inhibition process, which, moreover, requires the two hemispheres to operate at the same time. Within-hemisphere preparation (hand-cues), on the other hand, specifies relevant responses located in one hemisphere and irrelevant responses located in the other hemisphere. This clear neural separation of relevant and irrelevant responses represented in different hemispheres allows a rather global and strong process of response inhibition concentrated in one hemisphere. In this view, eliciting extra activation in both hemispheres by moving the hands was expected to decrease the within-hemisphere inhibition advantage. RT results showed that 
moving the hands eliminated the hand-advantage, thereby supporting the idea that activating both hemispheres by moving the hands may counteract a response inhibition process that operates more efficiently on response alternatives grouped together in one hemisphere than on response alternatives distributed across two hemispheres.

In the final study (Chapter 6; Moresi et al., 2009), we used "aging" as a vehicle to test the automatic vs. effortful processing assumption. If, as many studies have demonstrated, advancing age is accompanied by a reduction in central, effortful processes while leaving automatic processes intact (e.g., Allen et al., 1993; Ford et al., 1997; Wishart et al., 2000), then finger- and neither-cue (RT) benefits should be severely affected by older age whereas hand-cue benefits should be relatively spared. Indeed, RT results showed an age-related deficit in the speed of between-hands preparation, not in the speed of within-hand preparation.

\section{Future research and conclusion}

In the following sections, we discuss new directions for research on the finger cuing task in relation to response preparation as well as possible applications of the task in clinical and non-clinical settings.

\section{Strategies}

A striking outcome of the cue validity study (Chapter 4; Moresi et al., 2008a) was that all participants switched between two pupil dilation strategies: an "early" strategy, characterized by an initial increase in pupil dilation that started soon after cue onset, and a "late" strategy, characterized by an initial decrease in pupil dilation at cue onset followed by an increase that was linked with the stimulus onset. Averaged over all trials and all participants, both pupil strategies in this study occurred about equally often. Interestingly, a follow-up analysis on the occurrence of these two strategies in our first study that used the standard ( $100 \%$ valid cues) finger-cuing task (Chapter 2 ; Moresi et al., 2008b) showed that the "early" strategy occurred on about $90 \%$ of all trials). Hence, cue validity seems to be an important determinant of the relative occurrence of both strategies, with invalid cues promoting the switch from an early to a late strategy. Future research should provide more insight on when these pupil strategies occur and why. A suggestion would be to systematically manipulate cue validity and to determine how this factor influences the incidence of both strategies. Assuming that a late strategy is associated with an increased focus on stimulus processing (at the expense of 
cue processing), one might expect a systematic increase in the "late" strategy as cue validity decreases. Future studies might also consider the issue of pupil dilation strategy in the context of different presentation modes of the preparation intervals (i.e., blocked versus random). This would be especially relevant in the context of the finding that the pattern of differential cuing benefits in older age disappears with randomized but not with blocked preparation intervals (see Chapter 6).

Response inhibition and heart rate

Response inhibition influences heart rate. The interval between successive heart beats is prolonged when a motor response is successfully inhibited compared to when inhibition fails (De Jong, Coles, \& Logan, 1995). Full and partial response inhibition both lead to a similar slowing of heart rate, which suggests that one mechanism controls inhibition in both situations (Van der Veen, Van der Molen, \& Jennings, 2000). The reason why heart rate and response inhibition interact is that both are controlled by the same brain areas, being the cerebellum and the basal ganglia (De Jong et al., 1995). The hypothesis that the hand-advantage might at least partially be related to response inhibition (see Adam \& Moresi, 2007; Chapter 5) could be further examined by studying heart rate slowing in the finger-cuing paradigm. In this view, unimanual hand-based response preparation should be associated with greater cardiac slowing than bilateral finger-based preparation.

Aging and $f M R I$

In a developmental study, Adam, Parthoens, and Pratt (2006) demonstrated that the brain mechanisms underlying hand-based response preparation mature much earlier than the brain mechanisms underlying finger-based response preparation. Their RT results indicated that hand-cued preparation benefits were independent of age group, while finger- and neither-cued benefits showed distinct developmental trends (with a 1-s preparation interval). In particular, hand-cues were already effective at the age of 7 years, finger-cues were effective at the age of 13 years, and neither-cues were effective at the age of 21 years. Thus, cue effectiveness co-varied with speed of development, with mechanisms mediating increasingly more difficult cues maturing at progressively later ages. This finding suggests a limited involvement of prefrontal areas in the processing of hand-cues because these areas mature relatively late (Stuss, 1992; Van der Molen, 2000). Interestingly, a reverse pattern is seen when people age (Adam et al., 1998). That is, where advancing age does not affect the ability to prepare hand-cues, older people (70-75 years) do encounter great difficulty using finger- and neither-cues. This 
fits with the age-related neurodegeneration of the prefrontal cortex (e.g., Tisserand \& Jolles, 2003).

To determine the neural mechanisms of the RT advantage for hand-cues, Adam et al. (2003a) conducted an fMRI study on the brain activation patterns involved in finger cuing. The fMRI data demonstrated brain activation in the prefrontal cortex, parietal cortex, and basal ganglia. However, the prefrontal cortex showed no significant differences across the hand-, finger-, and neither-cued conditions. This was rather unexpected because developmental (Adam et al., 2006) and aging studies (Adam et al., 1998) suggest strong differential involvement of the prefrontal cortex in finger preparation. To further investigate prefrontal cortex activity in the finger-cuing task, we suggest extending the preparation interval. In the original fMRI study (Adam et al., 2003a) this interval was relatively short $(500 \mathrm{~ms})$, which may have prevented the full and complete engagement of the necessary top-down processes to prepare for the more difficult finger- and neither-cues. This possibility is consistent with the finding that the precuing benefit in these conditions was significantly smaller than that in the hand-cued condition.

The finger-cuing task as a diagnostic tool of executive dysfunction Given its multi-dimensional nature, the finger-cuing task may prove valuable in applied and clinical settings. In particular, the finger-cuing task allows the separate assessment of the quality of two distinct preparatory mechanisms, namely fast automatic preparation (indexed by hand-cued benefits) and slow effortful preparation (indexed by finger- and neither-cued benefits). In addition, manipulation of the preparation interval, both in duration and presentation mode (blocked vs. random), may offer the opportunity to investigate the processes associated with temporal preparation, that is, the ability to optimize the timing of preparation. For example, in a recent study on finger preparation in children with ADHD (Hurks, Adam, Hendriksen, Vles, Feron, \& Kalff, 2005), it was found that ADHD children were selectively impaired in their ability to engage in effortful, controlled preparation activities compared to healthy control children, whereas fast, automatic preparation was not affected.

The planning and preparing of an action (finger response) in the finger-cuing task, and the inhibition of non-relevant or invalid responses, are so-called "executive functions." These refer to cognitive abilities that control and regulate behavior (e.g., planning, inhibitory control, response preparation, and memory). Obviously, executive functions are important for successful 
adaptive behavior, because they allow people to plan, initiate, and complete tasks, but also to suppress unwanted actions. Given the similarity and overlap between executive functions and the cognitive processes involved in finger preparation, the finger-cuing task may be useful as a diagnostic tool to detect certain deficiencies in executive dysfunction and to evaluate the effectiveness of training or intervention programs.

Intelligence

In line with the suggestion made by several authors that motor and intellectual abilities might be related (e.g., Fuster, 2003; Keele, 2002), it would be interesting to examine the relationship between adaptive motor behavior and general intelligence (IQ) using the finger-cuing paradigm. According to Fuster (2003), intelligence is the processing of information towards cognitive and behavioral goals. It is characterized by the ability to adjust to changes, to solve new problems, and to create new forms of action. If we assume that intelligence includes the ability to create new forms of action, the Grouping Model would predict large IQ-related individual differences in planning behavior for the difficult, bilateral finger- and neither-cues, but not, or to a lesser degree, for the easy, unilateral hand-cues. We have found preliminary evidence in support of this prediction in a recent pilot study, which compared performance on the finger-cuing task between two groups of participants that differed in intelligence. Follow-up studies could be combining performance measures (RT) with pupillometry. According to the Grouping Model, it is expected that highly intelligent individuals require less cognitive resources (as indexed by the cognitive pupillary response) to prepare the difficult finger- and neither-cues cues compared to less intelligent individuals. Handcues, on the other hand, are not expected to show an IQ-related effect.

\section{Conclusion}

In the present studies we used the cognitive pupillary response and RT to test two critical features of the Grouping Model of finger preparation, namely the grouping and the processing assumption. The combined results provided independent and converging evidence for these assumptions, thereby substantially strengthening the Grouping Model's empirical foundation. In particular, RT results revealed a strong dissociation between the preparatory processes involved in within- and between-hands preparation, because they were differentially influenced by the "moving hands" and "age" manipulations. Similarly, pupillometric results revealed a differential sensitivity of these two types of preparation for the "overlapping hands" and "cue validity" manipulations. These outcomes support the Grouping Model's 
notion that different neural processes modulate within- and between-hands preparation in the finger-cuing paradigm. In particular, the findings accord with the idea that between-hands preparation relies on fast, automatic, bottom-up processes, whereas within-hands preparation requires slower, effortful, top-down processes to establish a selective set. Finally, it is argued that the finger-cuing task, given its multifaceted nature and solid empirical and theoretical footing, may hold promise as a diagnostic tool for detecting, monitoring, and evaluating executive dysfunction in applied and clinical settings.

\section{REFERENCES}

Adam, J. J., Backes, W., Rijcken, J., Hofman, P., Kuipers, H., \& Jolles, J. (2003a). Rapid visuomotor preparation in the human brain: a functional MRI study. Brain Research: Cognitive Brain Research, 16(1), 1-10.

Adam, J. J., Hommel, B., \& Umilta, C. (2003b). Preparing for perception and action (I): the role of grouping in the response-cuing paradigm. Cognitive Psychology, 46(3), 302-358.

Adam, J. J., Hommel, B., \& Umiltà, C. (2005). Preparing for perception and action (II) Automatic and effortful Processes in Response cuing. Visual Cognition, 12(8), 1444-1473.

Adam, J. J., \& Moresi, S. (2007). Response preparation with static versus moving hands. Brain Cognition, 65(3), 252-259.

Adam, J. J., Paas, F. G., Teeken, J. C., van Loon, E. M., van Boxtel, M. P., Houx, P. J., et al. (1998). Effects of age on performance in a finger-precuing task. Journal of Experimental Psychology Human Perception and Performance, 24(3), 870-883.

Adam, J. J., Parthoens, S., \& Pratt, J. (2006). Distinct mechanisms for planning keypress and reaching responses: a developmental study. Human Movement Science, 25(3), 293-309.

Allen, P. A., Groth, K. E., Weber, T. A., \& Madden, D. J. (1993). Influence of response selection and noise similarity on age differences in the redundancy gain. Journal of Gerontology, 48(4), P189198.

De Jong, R., Coles, M. G., \& Logan, G. D. (1995). Strategies and mechanisms in nonselective and selective inhibitory motor control. Journal of Experimental Psychology Human Perception and Performance, 21(3), 498-511.

Dechent, P., \& Frahm, J. (2003). Functional somatotopy of finger representations in human primary motor cortex. Human Brain Mapping, 18(4), 272-283.

Ford, J. M., Roth, W. T., Isaacks, B. G., Tinklenberg, J. R., Yesavage, J., \& Pfefferbaum, A. (1997). Automatic and effortful processing in aging and dementia: event-related brain potentials. Neurobiology in Aging, 18(2), 169-180.

Fuster, J. M. (2003). Cortex and Mind - unifying cognition. New York: Oxford University Press.

Hurks, P. P., Adam, J. J., Hendriksen, J. G., Vles, J. S., Feron, F. J., Kalff, A. C., et al. (2005). Controlled visuomotor preparation deficits in attention-deficit/hyperactivity disorder. Neuropsychology, 19(1), 66-76.

Keele, S. (2002). An Interview with Stephen Keele. In M. S. Gazzaniga, R. B. Ivry \& G. R. Mangun (Eds.), Cognitive Neuroscience - The biology of the mind (pp. 474).

Moresi, S., Adam, J. J., Rijcken, J., \& Van Gerven, P.W.M. (2008a). Cue validity effects in response preparation: A pupillometric study. Brain Research, 1196, 94-102. 
Moresi, S., Adam, J. J., Rijcken, J., Van Gerven, P.W.M., Kuipers, H., \& Jolles, J. (2008b). Pupil dilation in response preparation. International Journal of Psychophysiology, 67(2), 124-130.

Moresi, S., Adam, J. J., Rijcken, J., Van Gerven, P.W.M., Werrij, B.G., van Boxtel, M. \& Jolles, J. (in press). Preparing Fingers within and between hands: Examining the maximal preparation benefit in older age. European Journal of Cognitive Psychology.

Reeve, T. G., \& Proctor, R. W. (1984). On the advance preparation of discrete finger responses. Journal of Experimental Psychology Human Perception and Performance, 10(4), 541-553.

Reeve, T. G., \& Proctor, R. W. (1985). Nonmotoric Translation Processes in the Preparation of Discrete Finger Responses: A Rebuttal of Miller's (1985) Analysis. Journal of Experimental Psychology: Human Perception and Performance., 11(2), 234-241.

Reeve, T. G., \& Proctor, R. W. (1990). The salient-features coding principle for spatial-and symboliccompatibility effects. In R. W. Proctor \& T. G. Reeve (Eds.), Stimulus-response compatibility (pp. 163-180). Amsterdam: North-Holland.

Stuss, D. T. (1992). Biological and psychological development of executive functions. Brain Cognition, 20(1), 8-23.

Tisserand, D. J., \& Jolles, J. (2003). On the involvement of prefrontal networks in cognitive ageing. Cortex, 39, 1107-1128.

Van der Molen, M. W. (2000). Developmental changes in inhibitory processing: evidence from psychophysiological measures. Biological Psychology, 54(1-3), 207-239.

van der Veen, F. M., van der Molen, M. W., \& Jennings, J. R. (2000). Selective inhibition is indexed by heart rate slowing. Psychophysiology, 37(5), 607-613.

Wishart, L. R., Lee, T. D., Murdoch, J. E., \& Hodges, N. J. (2000). Effects of aging on automatic and effortful processes in bimanual coordination. Journal of Gerontology Series B Biological, Psychological Sciences and Social Sciences, 55(2), P85-94. 


\section{SUMMARY \\ SAMENVATTING \\ CURRICULUM VITAE \\ WOORDEN VAN DANK \\ PUBLICATIONS}

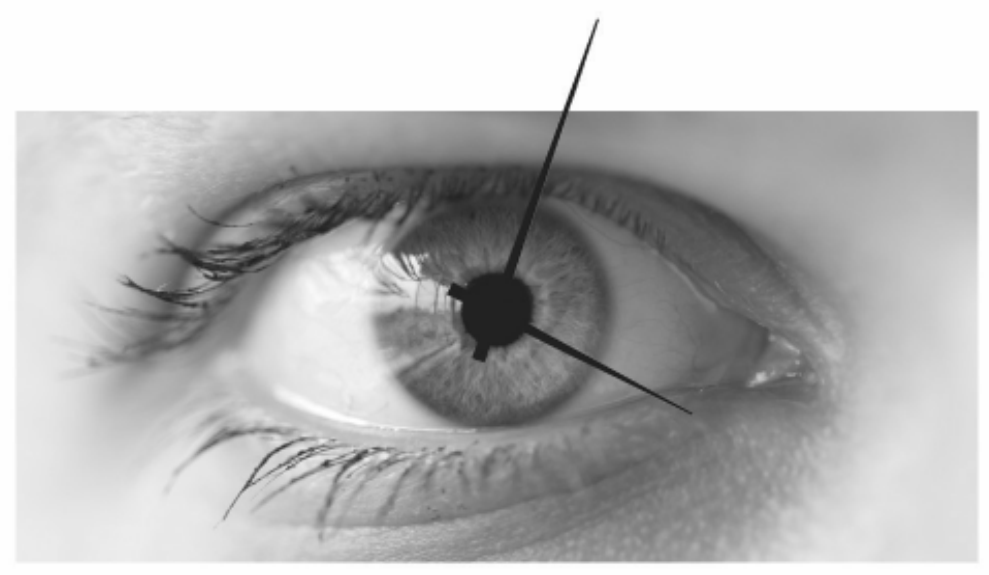




\section{SUMMARY}

In daily life, people often make use of visual cues. A good example of a cue is the orange traffic light, which prepares one either to move or to stop. Thus, a traffic light turning orange may stimulate preparatory activities, which will allow car drivers to rapidly detect the forthcoming red light and, if necessary, to stop the car immediately. In general, people perform faster when they are presented with a cue that indicates a future event. This thesis focuses on the preparatory processes that enable such a speeded action. In particular, we examined preparatory processes that underlie the hand-advantage, a robust phenomenon in finger-cuing studies. This phenomenon is the reaction time (RT) benefit that occurs when preparing two fingers on one hand as opposed to preparing two fingers on two hands. A recent account of the handadvantage is the 'Grouping Model' of finger preparation (Adam et al., 2003b, 2005), which is based on two assumptions, the 'grouping' and the 'processing' assumption. The current thesis contains five studies that evaluated these assumptions.

Chapter 1 describes the hand-advantage in more detail. This RT benefit arises in the so called 'finger-cuing' task. In this task, participants are presented with four horizontally arranged squares (stimuli), which appear on the screen. Participants can react by pressing one of four spatially compatible keys of a keyboard with the index and middle fingers of one of the two hands. Typical in these kind of 'cuing' tasks is that first a cue appears, which gives information about which fingers to prepare for the eventual response. In this way, participants are able to respond faster. In this task, the cue colors two boxes grey. Hence, the cue transforms the basic four-choice task into a twochoice task. Following the cue signal, the target stimulus is presented by making one of the two grey boxes black, thus indicating the required finger response.

To test the two assumptions of the Grouping Model in the finger-cuing task, we measured two variables: reaction time (RT) and the 'cognitive pupil response'. The latter delivers information about the cognitive load of a certain task. When a task becomes more difficult, the pupil will show more dilation. For example if you experience this summary as very difficult, then your pupils are more dilated than when you read a simple magazine. A limitation of RT is 
that it represents only one moment of measurement while the cognitive pupil response is a continuous measurement.

Chapter 2 describes the first test of the processing assumption. In this study we investigated the differences in pupil size and RT during response preparation in the 'finger-cuing' task. Typical for this task is that easy and more difficult cues are presented and this results in a pattern of differential cue difficulty. The processing assumption emphasizes that the more difficult cues (indicating two fingers on two hands) require a slower, more effortful and controlled, so-called 'top-down' process to select the correct responses (fingers). On the other hand, easier cues (indicating two fingers on one hand), require a faster, more automatic, so-called 'bottom-up' process. As predicted by the processing assumption, selection and preparation of the more difficult cues led to larger pupil dilations, which suggested a higher cognitive load.

Chapter 3 presents a study that tested the grouping assumption. The grouping assumption underlines the existence of natural groups that determine the relation between stimuli and responses. This means that leftright cues, which evoke preparation of two fingers on one hand, are very effective because they indicate a strong perceptual stimulus group (two squares left or right) and a strong corresponding response group (left or right hand). In contrast, cues that represent two fingers on two hands belong to less well-defined groups. In other words, the anatomical left-right distinction of groups is absent in these cues. For this study, we disrupted the compatibility between the stimulus and response set by using an overlapped hand placement next to an adjacent hand placement. In the overlapped hand placement, the index and middle fingers of both hands were placed alternately on the response keys, which resulted in the following arrangement: right index, left middle, right middle, and left index finger. Performance in the overlapped hands condition was compared to the standard adjacent hand placement. Results showed longer RTs and larger pupil dilations for the overlapped than for the adjacent hand placement, reflecting an overall increase in cognitive processing load. Importantly, overlapping the hands negatively affected left-right cues the most, while these cues were most efficient in the standard hands adjacent position. This specifies a higher cognitive load when preparing two fingers on one hand in the overlapped hand position. Our outcome supports the notion that the anatomical hand distinction is an important determinant of finger-cuing efficiency. This conclusion also supports the Grouping Model, which highlights the role of motoric factors in response preparation. 
Chapter 4 reports a study in which the validity of the cue is manipulated to provide another test of the processing assumption. This means that $75 \%$ of the cues were valid and $25 \%$ of the cues were invalid. Because invalid cues incorrectly specify fingers, reprogramming operations are required to suppress incorrectly prepared responses and reactivate already suppressed ones. Consistent with this assumption, overall, pupil dilations were overall stronger for invalid than for valid cues. Furthermore, reprogramming operations were especially effortful in preparing two fingers on one hand, because the reprogramming of 'automatic bottom-up' processes (two fingers on one hand) requires more effort than the correction of slow, 'effortful topdown' processes (two fingers on two hands). These findings are consistent with the processing assumption. Moreover, our results are compatible with studies, which examined 'inhibition'. These inhibition studies suggest that preparing two fingers on one hand is associated with stronger basal ganglia activity, suggesting stronger inhibition of irrelevant responses grouped together in one hemisphere (left or right).

Chapter 5 investigates the potential role of inhibition in finger preparation. For that purpose, a moving-hands response set was compared to the standard static-hands response set. It was assumed that the advantage of preparing two fingers on one hand (represented in one hemisphere) as opposed to preparing two fingers on two hands (represented in two hemispheres) is partly due to a response inhibition process that operates more efficiently within than between hemispheres. In this view, eliciting extra activation in both hemispheres by moving the hands decreased the within-hemisphere inhibition advantage. Translated to the processing assumption, these outcomes indicate that moving the hands only influences the automatic, bottom-up processes but not the controlled top-down processes.

Chapter 6 describes the last test of the processing assumption by examining if finger response preparation is sensitive to aging. If, as many studies have demonstrated, advancing age is accompanied by a reduction in controlled, effortful processes while leaving automatic processes intact, then preparing the more difficult cues (two fingers on two hands) should be affected by older age, whereas preparing the more easy cues (two fingers on one hand) should be relatively spared. RT results showed that elderly are capable of maximally preparing the difficult cues but they needed more time than youngsters. Thus, the age-related deficit is present in the speed of between-hands 
preparation, not in the speed of within-hand preparation, which is in line with the processing assumption.

Chapter 7 summarizes the most important outcomes of the previous chapters and puts them in a broader perspective. Together, the results of the above five studies provided independent and converging evidence for the two assumptions of the Grouping Model of finger preparation. These outcomes support the Grouping Model's notion that different processes modulate within- and between-hands preparation. In particular, the findings are in accordance with the idea that between-hands preparation relies on fast, automatic, bottom-up processes, whereas within-hands preparation requires slower, effortful, top-down processes to establish a selective set. Furthermore, it is argued that the finger-cuing task can be very valuable in applied and clinical settings. For example, it may hold promise as a diagnostic tool for detecting, monitoring, and evaluating executive dysfunctions, which are often associated with controlled processes. 


\section{SAMENVATTING}

In het dagelijkse leven maken we vaak gebruik van visuele aanwijzingen. Bij experimenteel psychologisch onderzoek spreekt men in dit verband over 'cues'. Een goed voorbeeld van een cue is het oranje verkeerslicht, dat mensen voorbereidt om te remmen of juist extra gas te geven voordat het licht op rood springt. Met andere woorden: het oranje verkeerslicht zorgt ervoor dat het rode licht sneller gedetecteerd wordt en, indien nodig, de auto onmiddellijk gestopt kan worden. Over het algemeen geldt dat mensen sneller reageren wanneer de cue aan een actie voorafgaat. Dit proefschrift concentreert zich op de voorbereidende processen die zulke versnelde reacties mogelijk maken. De term die hiervoor gehanteerd wordt, is responspreparatie. In het bijzonder ligt de focus van het proefschrift op de responspreparatieprocessen die het zogenaamde handvoordeel ondersteunen, een robuust fenomeen in het onderzoek naar het prepareren van vingerresponsen. Het handvoordeel is een reactietijdvoordeel dat ontstaat wanneer twee vingers op één hand geprepareerd worden in plaats van twee vingers op twee handen. Een recente verklaring voor dit handvoordeel komt voort uit het zogenaamde 'Grouping Model' (Adam et al., 2003, 2005). Dit model is gebaseerd op twee assumpties (aannames), namelijk de 'grouping'- en de 'processing'-assumptie. Dit proefschrift omvat vijf studies waarin de cognitieve pupilrespons en reactietijd (RT) gebruikt zijn om deze twee assumpties te testen.

Hoofdstuk 1 gaat nader in op het handvoordeel. Dit reactietijdvoordeel doet zich voor in de zogenaamde 'finger-cuing'-taak. In deze computertaak zien proefpersonen vier horizontaal geplaatste vierkantjes (stimuli) op het scherm verschijnen. De respons wordt gegeven door het indrukken van één van vier overeenkomstige toetsen van een toetsenbord met de wijs- of middelvinger van één van de twee handen. Typisch bij dit soort 'cuing'-taken is dat eerst een cue verschijnt die informatie geeft over de mogelijke responsen, zodat proefpersonen zich kunnen voorbereiden en daardoor sneller kunnen reageren. De cue in deze taak kleurt twee vierkantjes grijs en geeft zo informatie over twee responsmogelijkheden. Met andere woorden: de cue reduceert de vierkeuzemogelijkheid tot een tweekeuzemogelijkheid en daardoor kan de proefpersoon sneller reageren. Na de cue verschijnt de stimulus: één van de twee 'ge-cue-de' vierkantjes kleurt zwart. Deze geeft aan welke vingerrespons uiteindelijk moet worden uitgevoerd. 
Om de twee assumpties van het Grouping Model te testen met de fingercuing-taak, werden twee variabelen gemeten. Allereerst de reactietijd (RT).Dit is de tijd tussen de stimulus en de respons. Daarnaast werd de zogenaamde 'cognitieve pupilrespons' gemeten. Deze levert informatie over de cognitieve belasting van een taak. Wanneer een taak moeilijker wordt, zal de pupil meer verwijden. Bijvoorbeeld als u deze samenvatting als moeilijk ervaart, zal uw pupil groter zijn dan wanneer u een eenvoudig tijdschrift leest. De beperking van een RT is dat deze slechts één meetmoment representeert. De 'cognitieve pupilrespons' kan echter continu gemeten worden.

Hoofdstuk 2 beschrijft de eerste test van de processing-assumptie. In deze studie werd gekeken naar de verschillen in pupilgrootte en RT tijdens responspreparatie in de finger-cuing-taak. Typisch aan deze taak is dat makkelijke en moeilijke cues worden aangeboden en dit resulteert in een patroon van differentiële cue-moeilijkheid. De 'processing'-assumptie zegt hierover dat het prepareren van moeilijkere cues (zoals twee vingers op twee handen) een trager, meer gecontroleerd, zogenaamd 'top-down'-proces vereist om de juiste responsen (vingers) te selecteren, terwijl het prepareren van makkelijkere cues (zoals twee vingers op één hand) een sneller, meer 'automatisch', zogenaamd 'bottom-up'-proces vereist. Zoals voorspeld door de 'processing'-assumptie, ging de preparatie en selectie van twee vingers op twee handen gepaard met een grotere pupilverwijding en dit suggereert een hogere cognitieve belasting.

Hoofdstuk 3 beschrijft een studie die de grouping-assumptie test. De grouping-assumptie benadrukt het bestaan van natuurlijke groepen, die de samenhang tussen stimuli en responsen bepalen. Hiermee kan worden voorspeld dat links-rechts-cues, die aanzetten tot het prepareren van twee vingers op één hand, zeer effectief zijn omdat ze een sterke perceptuele stimulusgroep (twee vierkantjes links of rechts) en een sterke corresponderende motorische responsgroep vormen (linker- of rechterhand). Dit in tegenstelling tot cues die twee vingers op twee handen representeren, die tot minder sterk gedefinieerde groepen behoren. Met andere woorden: bij dit soort cues is het anatomische links-rechtsonderscheid van groepen niet meer aanwezig. In deze studie werd de compatibiliteit tussen de stimulus- en responsset verstoord door de handen te 'kruisen' (over elkaar te leggen), waarbij de vingers de volgende volgorde aannamen: rechter wijsvinger, linker middelvinger, rechter middelvinger, linker wijsvinger. De prestatie met gekruiste handen werd vervolgens 
vergeleken met de prestatie in de standaardpositie (handen naast elkaar). De resultaten toonden een grotere pupilverwijding, die gepaard ging met een tragere RT bij gekruiste handen vergeleken met de standaard handpositie. Voornamelijk de links-rechts-cues werden het meest beïnvloed door het kruisen van de handen, terwijl deze cues in de standaard handpositie juist het meest efficiënt zijn. Dit duidt op een hogere cognitieve belasting bij het prepareren van twee vingers op één hand in de gekruiste handpositie. Deze uitkomst laat zien dat het anatomische links-rechts-onderscheid voor een belangrijk deel de efficiëntie van vingerresponspreparatie bepaalt. Dit resultaat suggereert dus een prominente rol van motorische factoren in responspreparatie, een rol die expliciet door het Grouping Model wordt erkend.

Hoofdstuk 4 beschrijft een studie waarin de validiteit (geldigheid) van de cue gemanipuleerd werd om de processing-assumptie verder te testen. Dat wil zeggen: in $75 \%$ van de gevallen waren de cues valide en in $25 \%$ van de gevallen waren de cues niet valide. Niet-valide cues geven foutieve informatie over welke vingers geprepareerd moeten worden en vereisen daarom, zodra de stimulus verschijnt, een herprogrammering om de juiste vingerrespons te kunnen selecteren. De respons op niet-valide cues was trager en de pupilverwijding groter, waaruit afgeleid werd dat herprogrammering tijd en moeite kost. Het prepareren van twee vingers op één hand bleek het gevoeligst voor niet-valide cues. Dit suggereert dat het herprogrammeren van automatische niet-valide cues (twee vingers op één hand) meer moeite kost dan het herprogrammeren van de 'meer gecontroleerde niet-valide cues (twee vingers op twee handen). Deze bevindingen zijn consistent met de processing-assumptie. Bovendien zijn ze consistent met het gegeven dat het prepareren van twee vingers op één hand samengaat met een sterke activiteit in de basale ganglia, die zorgt voor de inhibitie (het onderdrukken) van de niet relevante responsalternatieven die gegroepeerd zijn in één hemisfeer (links of rechts).

Hoofdstuk 5 beschrijft de potentiële rol van inhibitieprocessen bij het prepareren van vingerresponsen. Hiervoor werd een conditie waarin de handen bewogen werden vergeleken met een conditie waarin de handen statisch waren. Zoals vermeld in Hoofdstuk 4, verloopt het responsinhibitieproces mogelijkerwijs efficiënter binnen één hemisfeer dan tussen hemisferen. Door de handen te laten bewegen werd dit binnenhemisfeer-voordeel verminderd ten opzichte van de statische conditie, omdat door het bewegen van de twee handen beide hemisferen geactiveerd 
worden. Vertaald naar de 'processing'-assumptie, laten de bevindingen zien dat het bewegen van de handen enkel de automatische, bottom-upprocessen beïnvloedt, niet de meer gecontroleerde, top-down-processen.

Hoofdstuk 6 beschrijft een laatste test van de 'processing'-assumptie door te bestuderen of vingerresponspreparatie gevoelig is voor veroudering. Algemeen is bekend dat ouderdom de meer gecontroleerde, moeilijkere informatieverwerkingsprocessen aantast maar niet de automatische, snelle processen. In deze studie werd de preparatietijd verlengd tot $5 \mathrm{sec}$. om de oudere proefpersonen meer tijd te geven om te prepareren en om zo na te gaan of zij de moeilijkere cues (twee vingers op twee handen) toch konden prepareren. De resultaten lieten zien dat bij voldoende lange preparatietijden, ouderen alle cues even effectief konden prepareren. Dit suggereert dat ouderen in staat zijn om de moeilijke cues maximaal te prepareren, maar dat zij daar meer tijd voor nodig hebben dan jongeren. Het ouderdomsgerelateerde tekort zit hem dus in de snelheid van het prepareren van twee vingers op twee handen, maar niet in de snelheid van het prepareren van twee vingers op één hand. Dit komt overeen met de processing-assumptie.

Hoofdstuk 7 vat de belangrijkste bevindingen van alle voorafgaande hoofdstukken samen en plaatst deze in een breder kader. Allereerst leverden de resultaten uit de bovenstaande vijf studies onafhankelijk en convergerend bewijs voor de twee assumpties die voortkomen uit het Grouping Model van vingerresponspreparatie. Er was sprake van een sterke dissociatie tussen prepareren van twee vingers op één hand en prepareren van twee vingers op twee handen. Deze dissociatie stemt overeen met het idee dat preparatie via twee verschillende processen tot stand gebracht kan worden: via een traag, moeilijk top-down proces (twee vingers op twee handen) en via een snel, automatisch, bottom-up proces (twee vingers op één hand). Vervolgens werd geopperd dat het gebruik van de finger-cuing-taak waardevol kan zijn in toegepaste en klinische settings, bijvoorbeeld als diagnostisch instrument voor het detecteren, monitoren en evalueren van cognitieve disfuncties, die specifiek betrekking hebben op gecontroleerde processen. 


\section{WOORDEN VAN DANK}

\section{Promotoren,}

Dankbaarheid is het geheugen van het hart. (Hans Christian Andersen)

Welgemeende woorden van dank dat jullie mijn promotie hebben mogelijk gemaakt.

\section{Co-promotoren,}

Eenvoud is inzien wat je niet kunt weglaten. (Pierre Van Rossum)

Schrijven is een proces van jezelf beperkingen opleggen, opdat de essentie niet bedolven raakt onder een brij van woorden en zinnen. Jullie gaven me mooie voorbeelden. Bovendien was jullie enthousiasme en het streven naar perfectie voor mij steeds een bron van inspiratie.

\section{Collega's en stagiaires,}

Het einde kroont het werk.

Tot het einde te geraken was een hele klus. Zonder jullie had ik die niet geklaard.

\section{Vrienden,}

Vrienden zijn familieleden zonder bloedverwantschap. (Ronald Giphart)

Fijn dat jullie zoveel begrip toonden en er op ELK moment voor me waren!

\section{Familie,}

Als gij mij kunt verdragen, zal ik u gaarne zien. (Wim Helsen)

Mijn Belgische thuis, daar is het begin van mijn levensdraad aan vastgeknoopt. Na enkele jaren Nederland ben ik pas echt Belg geworden. Ik ben blij dat de afstanden tussen ons even klein zijn als geografisch gezien tussen Hoeselt, Maastricht en Landgraaf.

\section{Loek,}

De kunst van het leven is: thuis te zijn alsof men op reis is. (Godfried Bomans)

Het is zalig om met jou op reis te zijn door het leven en tegelijkertijd ook echt thuis te zijn. 


\section{CURRICULUM VITAE}

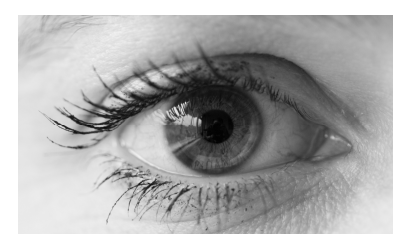

Sofie Moresi was born in Tongeren (Belgium) on July 7,1976 . She attended secondary school at the Institute Mariaburcht in Stevoort. After her graduation in 1994 she studied Physical Education and Biology at the Limburg Catholic University College in Hasselt. She completed her teaching degree in 1997 and started working as a teacher in a secondary school. In 1998, she decided to study the Master of Human Movement Sciences at Maastricht University (Netherlands). In her final year she combined this Master with the European Master of Physical Adapted Activity at the Catholic University of Leuven (Belgium). She chose for the specialty of sensomotory disorders and went to Exeter University (England) to do research on perceived competence in Cerebral Palsy children with Dr. Geoff Meek. During this time she also worked as a research assistant at the department of Sport Sciences. In September 2001 she finished both Masters. At the same month started lecturing at Bedford College and De Montford University in Bedford (United Kingdom). After one year of work experience she decided to extent her knowledge of life by exploring other continents beside Europe. Thereafter she got the opportunity to begin a PhD project, which was a collaboration of the Human Movement Sciences Department and the School of Mental Health and Neurosciences at Maastricht University. This project set off in November 2003 and resulted in this thesis. Currently Sofie is working as Lecturer-Researcher at the Fontys University of Applied Sciences in Sittard/ Tilburg (The Netherlands). 


\section{PUBLICATIONS}

Adam, J.J., \& Moresi, S. (2007). Response preparation with static versus moving hands. Brain Cognition, 65(3), 252-259.

Moresi, S., Adam, J. J., Gerven, P. W. M., Werrij, B. G., \& Van Boxtel, M. (In Press). Preparing fingers Within and Between Hands: Examining the maximal Preparation Benefit in Older Age. European Journal of Cognitive Psychology.

Moresi, S., Adam, J. J., Rijcken, J., Kuipers, H., \& Gerven, P. W. M. (submitted). Response Preparation with Adjacent versus Overlapped Hands: A Pupillometric Study

Moresi, S., Adam, J.J., Rijcken, J., \& Van Gerven, P.W.M. (2008). Cue Validity effects in Response Preparation: a pupillometric study. Brain Research, 1196, 94-102.

Moresi, S., Adam, J.J., Rijcken, J., Van Gerven, P.W.M., Kuipers, H., \& Jolles, J. (2008). Pupil dilation in Response Preparation. International Journal of Psychophysiology, 67(2), 124-130.

Roefs, A., Jansen, A., Moresi, S., Willems, P., van Grootel, S., \& van der Borgh, A. (2008). Looking good. BMI, attractiveness bias and visual attention. Appetite, 51(3), 552-555.

\section{Abstracts}

Moresi S., Adam, J.J. \& Gerven, P.W.M. (2005) Cognitive Pupil Responses in a Finger precuing Task. European Society for Cognitieve Psychology Conference. Leiden, The Netherlands.

Moresi S., Adam, J.J. \& Gerven, P.W.M. (2005) Pupil Responses in Motor Preparation.Task. Dutch Psychonomic Society Dutch Psychonomic Society Winterconference. Egmond aan Zee, The Netherlands.

Moresi S., Adam, J.J. \& Gerven, P.W.M. Pupil Responses in Motor Preparation. (2006) Task. Joined meeting of the Experimental Psychology Society \& Dutch Psychonomic Society. Birmingham, United Kingdom.

Moresi S., Adam, J.J. \& Gerven, P.W.M. Pupillary Responses in Motor Preparation. (2007) Brain \& Behavior Institute day. Maastricht, The Netherlands.

Moresi S., Adam, J.J., Rijken, J. \& Gerven P.W.M. (2007) Cue validity effects in Response Preparation. European Society for Cognitieve Psychology Conference. Marseille, France. 